\title{
Cold Trapped Ions as Quantum Information Processors
}

\author{
Marek Šašura and Vladimír Bužek \\ Research Center for Quantum Information, Slovak Academy of Sciences, Dúbravská cesta 9, Bratislava 842 28, Slovakia
}

(Dated: September 13, 2001)

In this tutorial we review physical implementation of quantum computing using a system of cold trapped ions. We discuss systematically all the aspects for making the implementation possible. Firstly, we go through the loading and confining of atomic ions in the linear Paul trap, then we describe the collective vibrational motion of trapped ions. Further, we discuss interactions of the ions with a laser beam. We treat the interactions in the travelling-wave and standing-wave configuration for dipole and quadrupole transitions. We review different types of laser cooling techniques associated with trapped ions. We address Doppler cooling, sideband cooling in and beyond the Lamb-Dicke limit, sympathetic cooling and laser cooling using electromagnetically induced transparency. After that we discuss the problem of state detection using the electron shelving method. Then quantum gates are described. We introduce single-qubit rotations, two-qubit controlled-NOT and multi-qubit controlled-NOT gates. We also comment on more advanced multi-qubit logic gates. We describe how quantum logic networks may be used for the synthesis of arbitrary pure quantum states. Finally, we discuss the speed of quantum gates and we also give some numerical estimations for them. A discussion of dynamics on off-resonant transitions associated with a qualitative estimation of the weak coupling regime and of the Lamb-Dicke regime is included in Appendix.

PACS numbers: 03.65.Ud, 03.67.Lx, 32.80.Pj, 32.80.Ys 


\section{Contents}

\section{Introduction}

III. Collective vibrational motion 6

A. Equilibrium positions 6

B. Normal modes 8

C. Quantized vibrational motion 10

IV. Laser-10n interactions 11

A. Travelling-wave configuration 13

B. Standing-wave contiguration 17

V. Laser cooling 19

A. Doppler cooling 19

B. Sideband cooling 20

1. Sideband cooling of two ions 21

2. Experimental sideband cooling 22

C. Sympathetic cooling 23

D. Laser cooling using electromagnetically induced transparency 25

VI. Electron shelving 26

VII. Quantum gates 26

A. Single-qubit rotations 27

B. Two-qubit controlled-NOT gates 27

C. Alternative implementation of two-qubit controlled-NO'T gates 29

1. Simplified quantum logic 29

2. Fast quantum gates 30

D. Multi-qubit controlled-NOT gates 30

E. Multi-qubit controlled- $R$ gates 31

VIII. Quantum logic networks 33

IX. Speed of quantum gates 35

X. Discussion 36

A. Decoherence 36

B. Ion trap systems 37

X1. Conclusion 38

Acknowledgments 38

A. Weak coupling regime 38

1. Off-resonant transitions 38

2. Stark light shifts 40

B. Lamb-Dicke regime 40

References 41 


\section{INTRODUCTION}

Although trapped ions have found many applications in physics [1], they caused a turning point in the evolution of quantum computing when the paper entitled Quantum computation with cold trapped ions was published by Cirac and Zoller in 1995 [2]. This proposal launched also an avalanche of other physical realizations of quantum computing using different physical systems, from high finesse cavities to widely manufactured semiconductors [3]. Through the years we have learnt a lot, but also revealed many peculiarities, about the physical realization of quantum computing which has led to many discussions concerning the conditions under which we could in principle implement quantum computing in certain quantum systems.

Before we give the list of requirements for the physical implementation of quantum computing we will introduce the fundamental terminology to appear throughout this paper. We will follow the definitions in Ref. 迎.

- A qubit is a quantum system in which the logical Boolean states 0 and 1 are represented by a prescribed pair of normalized and mutually orthogonal quantum states labelled as $|0\rangle$ and $|1\rangle$. These two states form a computational basis and any other (pure) state of the qubit can be written as a superposition

$$
|\psi\rangle=\alpha|0\rangle+\beta|1\rangle
$$

for some $\alpha$ and $\beta$ such that $|\alpha|^{2}+|\beta|^{2}=1$. It can be shown that we may choose $\alpha=\cos \vartheta$ and $\beta=e^{i \varphi} \sin \vartheta$. A qubit is typically a microscopic system, such as an atom, a nuclear spin or a polarized photon, etc. In quantum optics a two-level atom with a selected ground $|g\rangle$ and excited $|e\rangle$ state represents a qubit. Hence the notation $|g\rangle$ and $|e\rangle$ is used for the computational basis instead of $|0\rangle$ and $|1\rangle$. For instance, some qubits can serve for logic operations or the storage of information. Then we refer to logic qubits. Some others can be used especially for sympathetic cooling of logic qubits and we may call them cooling qubits. Some further qubits can be used as a quantum channel for transferring the information between distinct logic qubits and then we refer to them as to a quantum data bus.

- A quantum register of size $N$ refers to a collection of $N$ qubits.

- A quantum gate is a device which performs a fixed unitary operation on selected qubits in a fixed period of time.

- A quantum network is a device consisting of quantum gates whose computational steps are synchronized in time.

- A quantum computer (processor) can be viewed as a quantum network or a family of quantum networks.

- A quantum computation (computing) is defined as a unitary evolution associated with a set of networks which takes a initial quantum state (input) into a final quantum state (output) and can be interpreted in terms of the theory of information processing.

For the moment we presume that the following five requirements (termed DiVincenzo's checklist) should be met in order to realize quantum information processing on a quantum system [5]. Actually, there are two more requirements for the case of the transmission of qubits in space (flying qubits). However, it appears that all these requirements are necessary but not sufficient for successful experimental realization of a quantum processor [6].

(1) The system must provide a well characterized qubit and the possibility to be scalable in order to create a quantum register.

(2) We must be able to initialize a simple initial state of the quantum register.

(3) Quantum gate operation times must be much shorter than decoherence times. The quantum gate operation time is the period required to perform a certain quantum gate on a single qubit or on a set of qubits. The decoherence time approximately corresponds to the duration of the transformation which turns a pure state of the qubit $|\psi\rangle=\alpha|0\rangle+\beta|1\rangle$ into a mixture $\hat{\rho}=|\alpha|^{2}|0\rangle\left\langle\left. 0|+| \beta\right|^{2} \mid 1\right\rangle\langle 1|$.

(4) We need a set of quantum gates, to perform any unitary evolution operation that can be realized on the quantum system. It has been shown that any unitary evolution can be decomposed into a sequence of single qubit rotations and two-qubit controlled-NOT (CNOT) gates [7].

(5) The result of a quantum computational process must be efficiently read out, i.e. the ability to measure distinct qubits is required. 
Now we introduce briefly the physical system under consideration. Cold trapped ions is a quantum system of $N$ atomic ions confined in a linear trap. We assume an anisotropic and harmonic trapping potential. The ions are laser cooled to a very low temperature, beyond the Doppler cooling limit, reaching the recoil cooling limit [8]. Hence the term cold trapped ions. The ions form a linear crystal and oscillate in vibrational collective motional modes around their equilibrium positions. In their internal structure, depending on the choice of atomic species, we distinguish distinct atomic levels. The ions are individually addressed with a laser or a set of lasers in the travelling-wave or standing-wave configuration. We can detect the internal state of ions using optical detection devices. Further, we address briefly the requirements for the physical implementation of quantum computing (mentioned above) using cold trapped ions.

(1) The qubit is represented by a selected pair of internal atomic states denoted as $|g\rangle$ and $|e\rangle$. This selection is discussed in detail in Sec. IV. The quantum register is realized by $N$ ions forming the ion string in the linear trap, namely the linear Paul trap, which is reviewed in Sec. II. A selected collective vibrational motional mode (normal mode) is used as the quantum data bus. The vibrational motion of the ions is treated in Sec. III.

(2) Different laser cooling techniques can be used for the proper initialization of the motional state of the ions. They are described in Sec. V. The initial internal state where all the ions are in the state $|g\rangle$ can be reached by optical pumping to atomic states fast decaying to the ground state $|g\rangle$ (Sec. $\mathrm{V}$ and $\mathrm{VI}$ ).

(3) The influence of the decoherence on the motional state of the ions is suppressed by laser cooling to ground motional states of the normal modes. The internal levels of the ions representing the qubit states $|0\rangle$ and $|1\rangle$ are selected such that they form slow transitions with excited states of long lifetimes. A very detailed discussion of the decoherence bounds of trapped atomic ions can be found in Ref. 22].

(4) Single-qubit quantum rotations can be realized on any ion and two-qubit controlled-NOT and multi-qubit controlled-NOT quantum gates can be applied between chosen ions due to the possibility of individual addressing with laser beams. The implementation of quantum gates is discussed in Sec. VII.

(5) The result of a computational process on cold trapped ions is encoded into the final state of the internal atomic states. This information can be very efficiently read out using the electron shelving method addressed in Sec.VI.

\section{ION TRAPPING}

Due to the charge of atomic ions, we can confine them by particular arrangements of electromagnetic fields. For studies of ions at low energy two types of traps are used. (i) Penning trap uses a combination of static electric and magnetic fields and (ii) Paul trap confines ions by oscillating electric fields. Paul was awarded the Nobel Prize in 1990 for his work on trapping particles in electromagnetic fields [9]. The operation of different ion traps is discussed in detail in Ref. [10]. For the purpose considered in this paper we will discuss only one trap configuration: the linear Paul trap (FIG.11). We will follow Ref. [10] and [1] for the mathematical treatment.

The linear Paul trap is basically a quadrupole mass filter, which is plugged at the ends with static electric potentials. An electric potential

$$
\phi_{0}=U_{0}+V_{0} \cos (\Omega t)
$$

oscillating with the radiofrequency $\Omega$ is applied between two diagonally opposite rod electrodes. The electrodes are coupled together with capacitors so that the potential (2.1) is constant as a function of the $z$ coordinate. The other two rod electrodes are grounded. The resulting potential at the trap axis (parallel with the $z$ direction) has the form

$$
\phi=\frac{\phi_{0}}{2 r_{0}^{2}}\left(x^{2}-y^{2}\right)=\frac{U_{0}+V_{0} \cos (\Omega t)}{2 r_{0}^{2}}\left(x^{2}-y^{2}\right),
$$

where $r_{0}$ is the distance from the trap centre to the electrode surface. In this field the (classical) equations of motion for an ion of the mass $m$ and charge $q$ are

$$
m \ddot{\mathbf{r}}=q \mathbf{E}=-q \nabla \phi, \quad \mathbf{r}=(x, y, z),
$$

or rewritten in the components

$$
\begin{aligned}
& \ddot{x}+\frac{q}{m r_{0}^{2}}\left[U_{0}+V_{0} \cos (\Omega t)\right] x=0, \\
& \ddot{y}-\frac{q}{m r_{0}^{2}}\left[U_{0}+V_{0} \cos (\Omega t)\right] y=0,
\end{aligned}
$$




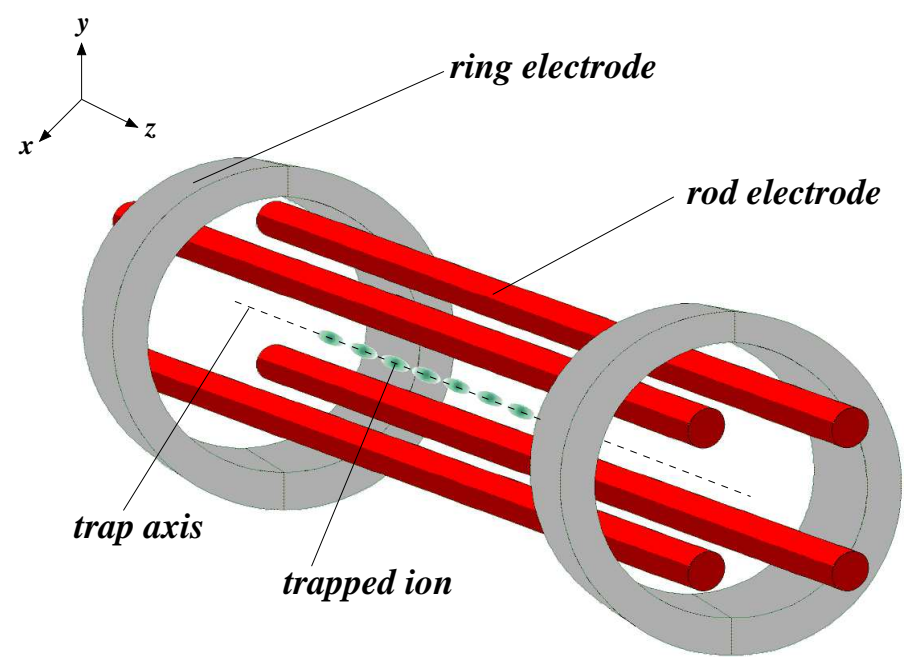

FIG. 1: Linear Paul trap in the configuration with two ring electrodes spaced by $2 z_{0}$. The diagonal distance between a pair of rod electrodes is $2 r_{0}$. Seven ions are confined at the trap axis. The potential (2.1) is applied between two diagonally opposite rod electrodes. The other two are grounded. Ideally, equal static potential is applied on both ring electrodes. Used by kind permission of Rainer Blatt [12].

After the substitution

$$
a=\frac{4 q U_{0}}{m r_{0}^{2} \Omega^{2}}, \quad b=\frac{2 q V_{0}}{m r_{0}^{2} \Omega^{2}}, \quad \zeta=\frac{\Omega t}{2},
$$

Eq. (2.4) and (2.5) take the form of the Mathieu equation

$$
\begin{aligned}
& \frac{d^{2} x}{d \zeta^{2}}+[a+2 b \cos (2 \zeta)] x=0 \\
& \frac{d^{2} y}{d \zeta^{2}}-[a+2 b \cos (2 \zeta)] y=0 .
\end{aligned}
$$

The Mathieu equation can be solved, in general, using the Floquet solution. However, typically we have $a \ll b^{2} \ll 1$, then the approximate stable solution of Eq. (2.8) and (2.9) are

$$
\begin{aligned}
& x(t) \approx x_{0}\left[1+\frac{b}{2} \cos (\Omega t)\right] \cos \left(\omega_{x} t+\varphi_{x}\right), \\
& y(t) \approx y_{0}\left[1-\frac{b}{2} \cos (\Omega t)\right] \cos \left(\omega_{y} t+\varphi_{y}\right),
\end{aligned}
$$

where

$$
\omega_{x}=\frac{\Omega}{2} \sqrt{\frac{b^{2}}{2}+a}, \quad \omega_{y}=\frac{\Omega}{2} \sqrt{\frac{b^{2}}{2}-a}
$$

and $x_{0}, y_{0}, \varphi_{x}, \varphi_{y}$ are constants determined by initial conditions. We see from Eq. (2.10) and (2.11) that the motion of a single trapped ion in the radial direction is harmonic with the amplitude modulated with the frequency $\Omega$. The harmonic oscillation corresponding to the frequencies $\omega_{x}$ and $\omega_{y}$ is called the secular motion, whereas the small contribution oscillating at $\Omega$ is termed the micromotion [13, 14]. We can eliminate the micromotion under certain conditions [14]. For instance, well chosen voltages on additional compensation electrodes (not shown in FIG. 11) null the micromotion. Then the ion behaves as if it was confined in a harmonic pseudopotential $\psi_{2 D}$ in the radial direction given by

$$
q \psi_{2 D}=\frac{m}{2}\left(\omega_{x}^{2} x^{2}+\omega_{y}^{2} y^{2}\right) .
$$

Typically, $U_{0}=0 \mathrm{~V}$ and hence $a=0$, so the radial frequencies $\omega_{x}$ and $\omega_{y}$ are degenerated. Then Eq. (2.13) reduces to

$$
q \psi_{2 D}=\frac{m \omega_{r}^{2}}{2}\left(x^{2}+y^{2}\right)
$$


where the radial trapping frequency $\omega_{r}$ is given by

$$
\omega_{r}=\frac{\Omega b}{2 \sqrt{2}}=\frac{q V_{0}}{m r_{0}^{2} \Omega \sqrt{2}} .
$$

In experiments 13, 14, 15, 16, 17, typical operating parameters are $V_{0} \simeq 300-800 \mathrm{~V}, \Omega / 2 \pi \simeq 16-18 \mathrm{MHz}$, $r_{0}=1.2 \mathrm{~mm}$, so we achieve the radial frequency $\omega_{r} / 2 \pi \simeq 1.4-2 \mathrm{MHz}$ for Calcium ions ${ }^{40} \mathrm{Ca}^{+}$. In nature, $97 \%$ of Calcium consists of this isotope. To provide confinement along the $z$ direction, static potentials $U_{1}$ and $U_{2}$ are applied on the ring electrodes. Ideally, $U_{1}=U_{2}=U_{12}$. Numerical calculations show that the potential near the trap centre at the trap axis is harmonic with the approximate axial trapping frequency $\omega_{z}$ given by

$$
\frac{1}{2} m \omega_{z}^{2} z_{0}^{2} \approx \xi q U_{12},
$$

where $z_{0}$ is the distance from the trap centre to the ring electrode and $\xi$ is a geometric factor describing how much of the static field from the ring electrodes is present along the trap axis 13. Typical parameters are $\omega_{z} / 2 \pi \simeq 500-700 \mathrm{kHz}$ for $U_{12} \simeq 2000 \mathrm{~V}$ and $z_{0}=5 \mathrm{~mm}$ [16, 17. The resulting pseudopotential for ions confined in the linear Paul trap in all three directions takes the form

$$
q \psi_{3 D}=\frac{m \omega_{r}^{2}}{2}\left(x^{2}+y^{2}\right)+\frac{m \omega_{z}^{2} z^{2}}{2},
$$

where the radial trapping frequency $\omega_{r}$ is given by Eq. (2.15) and the axial trapping frequency $\omega_{z}$ is defined by Eq. (2.16). For values of experimental parameters given above, we can calculate the depth of the potential well in the axial direction $\left(\omega_{z} / 2 \pi \simeq 700 \mathrm{kHz}\right)$

$$
V_{z}=\frac{m \omega_{z}^{2} z_{0}^{2}}{2} \simeq 100 \mathrm{eV}
$$

and in the radial direction $\left(\omega_{r} / 2 \pi \simeq 2 \mathrm{MHz}\right)$

$$
V_{r}=\frac{m \omega_{r}^{2} r_{0}^{2}}{2} \simeq 820 \mathrm{eV} .
$$

The potential well in the radial direction is almost several times deeper than along the trap axis, i.e. there is a strong binding in the radial direction. Therefore we will not take into account radial oscillations of the ions in our further considerations.

Finally we briefly mention how ions are loaded into the trap. We will follow the account of practical procedures in Ref. [14]. Before starting the loading process, the trapping potentials are turned off for a while in order to get rid of any unwanted trapped residual ions. The atomic oven producing Calcium atoms is switched on and heats up. This takes about a minute. Then we turn on the electron gun ionizing neutral Calcium atoms directly in the trapping volume. Cooling lasers are directed on the ion cloud containing several hundreds of ions with a diameter of about $200 \mu \mathrm{m}$. The ion cloud gradually relaxes into a steady state where the radiofrequency heating (from the electrodes) is balanced by laser cooling. The number of trapped ions is reduced by turning off the cooling. At low ion numbers, the ions undergo a phase transition and form a linear crystal structure. Therefore, we refer to the ion crystal or to the ion string or eventually to the ion chain. The loading process itself takes normally about a minute.

\section{COLLECTIVE VIBRATIONAL MOTION}

\section{A. Equilibrium positions}

We have learnt that the ions form a linear crystal structure in the linear Paul trap after the loading process. We will assume a string of $N$ trapped ions. Due to the strong binding we can neglect the radial oscillations. However, if a large number of ions is confined in the trap, the radial vibrations become unstable and the ions undergo a phase transition from a linear shape to an unstable zig-zag configuration. The relation

$$
\alpha_{c r i t}=c N^{\beta}
$$

determines a critical value for the ratio of the trapping frequencies $\alpha=\left(\omega_{z} / \omega_{r}\right)^{2}$ for a given number of trapped ions $N$. When $\alpha$ exceeds the critical value $\alpha_{\text {crit }}$, the ions are exposed to a zig-zag motion. The experimental values of the 
constants in Eq. 3.1) are $c \simeq 3.23$ and $\beta \simeq-1.83$. For experimental details and the theoretical treatment we refer to Ref. 18.

Further, we describe the collective vibrational motion of the ions. We will follow the treatment given by James in Ref. [20]. The ions are exposed to the harmonic potential (2.17) due to the trap electrodes and also to the repulsive Coulomb force from each other. Taking into account all the assumptions given above, the potential energy of $N$ ions confined in the linear Paul trap is given by the expression

$$
V=\sum_{i=1}^{N} \frac{m \omega_{z}^{2} z_{i}^{2}(t)}{2}+\sum_{\substack{i, j=1 \\ i<j}}^{N} \frac{q^{2}}{4 \pi \varepsilon_{0}} \frac{1}{\left|z_{i}(t)-z_{j}(t)\right|}
$$

where $z_{i}(t)$ is the position of the $i$ th ion numbering them from left to right with the origin in the trap centre, $m$ is the mass of the ion with the charge $q, \omega_{z}$ is the axial trapping frequency (2.16) and $\varepsilon_{0}$ is the permitivity of the vacuum.

Assuming that the ions are cold enough, we can write for the position of the $i$ th ion

$$
z_{i}(t)=\bar{z}_{i}+\Delta_{i}(t)
$$

where $\bar{z}_{i}$ is the equilibrium position and $\Delta_{i}(t)$ expresses small vibrations around $\bar{z}_{i}$. The ions placed in the equilibrium positions minimize the potential energy. Hence these positions are determined by the condition

$$
\left[\frac{\partial V}{\partial z_{i}}\right]_{\mathbf{z}=\overline{\mathbf{z}}}=0, \quad i=1, \ldots, N
$$

where $\mathbf{z}=\left(z_{1}, \ldots, z_{N}\right)$ and $\overline{\mathbf{z}}=\left(\bar{z}_{1}, \ldots, \bar{z}_{N}\right)$. We introduce a scaling factor $\gamma$ by the relation

$$
\gamma^{3}=\frac{q^{2}}{4 \pi \varepsilon_{0} m \omega_{z}^{2}}
$$

and the dimensionless equilibrium position as $\mathcal{Z}_{i}=\bar{z}_{i} / \gamma$. Then one can rewrite Eq. (3.4) to the form

$$
\mathcal{Z}_{i}-\sum_{j=1}^{i-1} \frac{1}{\left(\mathcal{Z}_{i}-\mathcal{Z}_{j}\right)^{2}}+\sum_{j=i+1}^{N} \frac{1}{\left(\mathcal{Z}_{i}-\mathcal{Z}_{j}\right)^{2}}=0, \quad i=1, \ldots, N
$$

$N=1$ is a trivial case $\left(\mathcal{Z}_{1}=0\right)$. We can find the analytical solution of Eq. (3.6) for two and three ions:

$$
\begin{array}{ll}
N=2, & \mathcal{Z}_{1}=-\sqrt[3]{1 / 4}, \quad \mathcal{Z}_{2}=\sqrt[3]{1 / 4} \\
N=3, & \mathcal{Z}_{1}=-\sqrt[3]{5 / 4}, \quad \mathcal{Z}_{2}=0, \quad \mathcal{Z}_{3}=\sqrt[3]{5 / 4}
\end{array}
$$

Numerical calculations are necessary for $N \geq 4$. For the Calcium ions ${ }^{40} \mathrm{Ca}^{+}$and the trap frequency $\omega_{z} / 2 \pi \simeq 700 \mathrm{kHz}$, we may calculate the equilibrium positions as

$$
\begin{aligned}
& N=2, \quad \Delta z_{\min } \simeq 7.7 \mu \mathrm{m}, \\
& N=3, \quad \Delta z_{\min } \simeq 6.1 \mu \mathrm{m} .
\end{aligned}
$$

The minimum value $\Delta z_{\text {min }}$ of the distance between two neighbouring ions in the trap occurs at the centre of the ion crystal, because the outer ions push the inner ions closer together. It has been calculated from numerical data that this minimum distance is given approximately by the relation [19, 20]

$$
\Delta z_{\min }(N) \approx \frac{2.018}{N^{0.559}} \gamma
$$

However, slightly different numerical results may be found in Ref. [22]. The relation (3.9) happens to be important when one considers individual ion addressing with a laser beam. Quantum statistics of the ion ensemble is not considered here because the spatial spread of the zeropoint wavefunctions of the individual ions is of the order of $10 \mathrm{~nm}$ and the wavefunction overlap is then negligible [19. 


\section{B. Normal modes}

The (classical) Lagrangian of the ions in the trap is given by the formula

$$
L \approx \frac{m}{2} \sum_{k=1}^{N} \dot{\Delta}_{k}^{2}-\frac{1}{2} \sum_{k, l=1}^{N}\left[\frac{\partial^{2} V}{\partial z_{k} \partial z_{l}}\right]_{\mathbf{z}=\overline{\mathbf{z}}} \Delta_{k} \Delta_{l},
$$

where we have expanded the potential energy $(3.2)$ in a Taylor series about the equilibrium positions. In the expansion we have omitted the constant term and the linear term which is zero [see Eq. (3.4)]. Higher order terms $\mathcal{O}\left(\Delta_{k}^{3}\right)$ have been also neglected. However, they may cause a cross-coupling between different vibrational modes which becomes a source of decoherence [22]. The partial derivatives in Eq. (3.10) can be calculated explicitly and we obtain the expression

$$
L=\frac{m}{2}\left(\sum_{k=1}^{N} \dot{\Delta}_{k}^{2}-\omega_{z}^{2} \sum_{k, l=1}^{N} V_{k l} \Delta_{k} \Delta_{l}\right)
$$

where

$$
V_{k l}=\frac{1}{m \omega_{z}^{2}}\left[\frac{\partial^{2} V}{\partial z_{k} \partial z_{l}}\right]_{\mathbf{z}=\overline{\mathbf{z}}}= \begin{cases}1+\sum_{\substack{j=1 \\ j \neq k}}^{N} \frac{2}{\left|\mathcal{Z}_{k}-\mathcal{Z}_{j}\right|^{3}}, & k=l, \\ -\frac{2}{\left|\mathcal{Z}_{k}-\mathcal{Z}_{l}\right|^{3}}, & k \neq l .\end{cases}
$$

It follows from Eq. (3.12) that $V_{k l}=V_{l k}$. The values of $\mathcal{Z}_{j}$ are given by Eq. (3.7) for $N=2$ and for $N=3$, whereas they have to be calculated numerically for $N \geq 4$.

The dynamics of the trapped ions is governed by the Lagrange equations

$$
\frac{d}{d t} \frac{\partial L}{\partial \dot{\Delta}_{k}}-\frac{\partial L}{\partial \Delta_{k}}=0, \quad k=1, \ldots, N
$$

with the Lagrangian given by Eq. (3.11). We will search for a particular solution of Eq. (3.13) in the form

$$
\Delta_{k}(t)=C_{k} e^{-i \nu t}, \quad k=1, \ldots, N,
$$

where $C_{k}$ are constants. Substituting Eq. (3.14) into (3.13) we get the condition for $\nu$ in the form

$$
\left\|\omega_{z}^{2} V_{k l}-\nu^{2} \delta_{k l}\right\|=0
$$

where $\delta_{k l}$ is the Kronecker symbol and $\|\ldots\|$ denotes the determinant. The equation (3.15) has in general up to $N$ real and nonnegative solutions $\nu_{\alpha}$. The frequencies $\nu_{\alpha}$ are characteristic parameters of the system. They depend only on its physical features (not on initial conditions). A general solution of Eq. (3.13) is a superposition of particular solutions (3.14) and we may write

$$
\Delta_{k}(t)=\sum_{\alpha=1}^{N} D_{k}^{(\alpha)} Q_{\alpha}(t), \quad k=1, \ldots, N,
$$

where

$$
Q_{\alpha}(t)=C_{\alpha} e^{-i \nu_{\alpha} t}
$$

By definition we will require the vectors

$$
\mathbf{D}^{(\alpha)}=\left(D_{1}^{(\alpha)}, \ldots, D_{N}^{(\alpha)}\right), \quad \alpha=1, \ldots, N
$$

to be the eigenvectors of the matrix $V_{k l}$ defined in Eq. (3.12), i.e.

$$
\sum_{k=1}^{N} V_{k l} D_{k}^{(\alpha)}=\mu_{\alpha} D_{l}^{(\alpha)}, \quad l, \alpha=1, \ldots, N,
$$


and also to be orthogonal and properly normalized

$$
\sum_{k=1}^{N} D_{k}^{(\alpha)} D_{k}^{(\beta)}=\delta_{\alpha \beta}, \quad \alpha, \beta=1, \ldots, N .
$$

We will number the eigenvectors in order of the increasing eigenvalues $\mu_{\alpha}$. It can be shown that the first two eigenvectors $(\alpha=1,2)$ always have the form

$$
\begin{aligned}
& \mathbf{D}^{(1)}=\frac{1}{\sqrt{N}}(1,1, \ldots, 1), \quad \mu_{1}=1, \\
& \mathbf{D}^{(2)}=\frac{1}{\sqrt{\sum_{k=1}^{N} \mathcal{Z}_{k}^{2}}}\left(\mathcal{Z}_{1}, \mathcal{Z}_{2}, \ldots, \mathcal{Z}_{N}\right), \quad \mu_{2}=3 .
\end{aligned}
$$

We should emphasize that Eq. (3.21) and (3.22) (they characterize two basic collective motional modes) are not dependent on the number $N$ of the ions in the trap. Next eigenvectors $(\alpha \geq 3)$ must be, in general, calculated numerically. Substituting Eq. (3.21) into (3.20) we get the relation

$$
\sum_{k=1}^{N} D_{k}^{(\alpha)}=0, \quad \alpha=2, \ldots, N .
$$

We can determine analytically the eigensystem for two and three ions:

$$
\begin{array}{rlrl}
N=2, & \mathbf{D}^{(1)}=\frac{1}{\sqrt{2}}(1,1), & \mu_{1}=1, \\
& \mathbf{D}^{(2)}=\frac{1}{\sqrt{2}}(-1,1), & \mu_{2}=3, \\
N=3, & \mathbf{D}^{(1)}=\frac{1}{\sqrt{3}}(1,1,1), & \mu_{1}=1, \\
\mathbf{D}^{(2)}=\frac{1}{\sqrt{2}}(-1,0,1), & \mu_{2}=3, \\
\mathbf{D}^{(3)}=\frac{1}{\sqrt{6}}(1,-2,1), & \mu_{3}=29 / 5 .
\end{array}
$$

For larger $N$, the eigenvectors and eigenvalues must be computed numerically. The numerical values for up to ten ions can be found in Ref. [20].

Substituting Eq. (3.16) into (3.11) we get a new expression for the Lagrangian

$$
L=\frac{m}{2} \sum_{\alpha=1}^{N}\left(\dot{Q}_{\alpha}^{2}-\nu_{\alpha}^{2} Q_{\alpha}^{2}\right),
$$

where

$$
\nu_{\alpha}=\omega_{z} \sqrt{\mu_{\alpha}} .
$$

The Lagrangian (3.27) has split into $N$ uncoupled terms, where $Q_{\alpha}$ [Eq. (3.17)] refer to the normal modes and $\nu_{\alpha}$ defined in Eq. (3.28) are termed the normal frequencies. Finally, the position of the $i$ th ion in the trap can be rewritten in terms of Eq. (3.16) using (3.3) to the form

$$
z_{i}(t)=\bar{z}_{i}+\Re\left\{\sum_{\alpha=1}^{N} C_{\alpha} D_{i}^{(\alpha)} e^{-i \nu_{\alpha} t}\right\}, \quad i=1, \ldots, N,
$$

where $\Re\{\ldots\}$ denotes the real part and $C_{\alpha}$ are constants given by initial conditions. The collective vibrational motion of trapped ions determined by the eigenvector $\mathbf{D}^{(1)}$ [Eq. (3.21]] refers to the normal mode called the center-of-mass (COM) mode

$$
z_{i}^{(1)}(t)=\bar{z}_{i}+\Re\left\{\frac{1}{\sqrt{N}} C_{1} e^{-i \omega_{z} t}\right\}, \quad i=1, \ldots, N
$$


and corresponds to all of the ions oscillating back and forth as if they were a rigid body. The motion determined by the next eigenvector $\mathbf{D}^{(2)}$, [Eq. (3.22] refers to the breathing mode

$$
z_{i}^{(2)}(t)=\bar{z}_{i}+\Re\left\{\frac{\bar{z}_{i}}{\sqrt{\sum_{k=1}^{N} \bar{z}_{k}^{2}}} C_{2} e^{-i\left(\omega_{z} \sqrt{3}\right) t}\right\}, \quad i=1, \ldots, N .
$$

It corresponds to each ion oscillating with the amplitude proportional to its equilibrium distance from the trap center. The COM motional mode can be excited in experiments by applying an additional AC voltage on one of the ring electrodes. For exciting the breathing motional mode, a 300-times higher voltage must be applied [15. Higher motional modes require gradient field excitation due to the nontrivial configuration of the ions in the ion string. However, in the limit of large ion trap dimension in comparison with the ion crystal dimension, the electrode electric fields are almost uniform across the ion crystal and the COM mode is very susceptible to heating due to these fields. Therefore, it seems to be more advantageous to use rather the breathing mode, which is much less influenced by uniform fields, as the quantum data bus. This will be discussed in more detail later on in the section on sympathetic cooling (Sec. $\mathrm{VQ}$ ). On the other hand, the ions can be easily addressed with a laser beam in the COM mode, while higher modes require accurate bookkeeping when addressing distinct ions in the ion crystal [20, 59].

\section{Quantized vibrational motion}

The normal modes $Q_{\alpha}$ are uncoupled in Eq. (3.27), so the corresponding canonical momentum conjugated to $Q_{\alpha}$ is $P_{\alpha}=m \dot{Q}_{\alpha}$ and one may write the (classical) Hamiltonian

$$
H=\frac{1}{2 m} \sum_{\alpha=1}^{N} P_{\alpha}^{2}+\frac{m}{2} \sum_{\alpha=1}^{N} \nu_{\alpha}^{2} Q_{\alpha}^{2} .
$$

The quantum motion of the ions can be considered by introducing the operators

$$
\begin{aligned}
Q_{\alpha} \rightarrow \hat{Q}_{\alpha} & =\sqrt{\frac{\hbar}{2 m \nu_{\alpha}}}\left(\hat{a}_{\alpha}^{\dagger}+\hat{a}_{\alpha}\right), \\
P_{\alpha} & \rightarrow \hat{P}_{\alpha}=i \sqrt{\frac{\hbar m \nu_{\alpha}}{2}}\left(\hat{a}_{\alpha}^{\dagger}-\hat{a}_{\alpha}\right)
\end{aligned}
$$

with the corresponding commutation relations

$$
\left[\hat{Q}_{\alpha}, \hat{P}_{\beta}\right]=i \hbar \delta_{\alpha \beta}, \quad\left[\hat{a}_{a}, \hat{a}_{\beta}^{\dagger}\right]=\delta_{\alpha \beta} .
$$

The Hamiltonian operator associated with the external (vibrational) degrees of freedom of the trapped ions is then expressed as follows $\left(H \rightarrow \hat{H}_{e x t}\right)$

$$
\hat{H}_{e x t}=\sum_{\alpha=1}^{N} \hbar \nu_{\alpha}\left(\hat{a}_{\alpha}^{\dagger} \hat{a}_{\alpha}+1 / 2\right)
$$

where $\hat{a}_{\alpha}$ and $\hat{a}_{\alpha}^{\dagger}$ are the usual annihilation and creation operators referring to the $\alpha$ th normal mode. We use the standard notation for the number states associated with the collective vibrational motion of the ions

$$
\hat{a}_{\alpha}^{\dagger} \hat{a}_{\alpha}\left|n_{\alpha}\right\rangle=n_{\alpha}\left|n_{\alpha}\right\rangle
$$

where $\left|n_{\alpha}\right\rangle$ refers to the state of the $\alpha$ th normal mode and $n_{\alpha}$ denotes the number of vibrational phonons in this mode. The states $\left\{\left|n_{\alpha}\right\rangle\right\}$ form the complete and orthonormal basis

$$
\left\langle m_{\alpha} \mid n_{\beta}\right\rangle=\delta_{\alpha \beta} \delta_{m n} .
$$

We can quantize the motion of the ions by applying Eq. (3.33) to the relation (3.16) and expressing the displacement operator of the $i$ th ion in the time-independent picture

$$
\hat{\Delta}_{i}=\sum_{\alpha=1}^{N} D_{i}^{(\alpha)} \sqrt{\frac{\hbar}{2 m \nu_{\alpha}}}\left(\hat{a}_{\alpha}^{\dagger}+\hat{a}_{\alpha}\right)=\sum_{\alpha=1}^{N} \mathcal{K}_{i}^{(\alpha)} z_{0}\left(\hat{a}_{\alpha}^{\dagger}+\hat{a}_{\alpha}\right), \quad i=1, \ldots, N,
$$


where [see Eq. (3.28)]

$$
\mathcal{K}_{i}^{(\alpha)}=\frac{D_{i}^{(\alpha)}}{\sqrt[4]{\mu_{\alpha}}}, \quad z_{0}=\sqrt{\frac{\hbar}{2 m \omega_{z}}} .
$$

We can easily calculate from Eq. (3.21) that for the COM mode applies

$$
\mathcal{K}_{i}^{(1)}=\frac{1}{\sqrt{N}}
$$

and for the breathing mode [Eq. (3.22)]

$$
\mathcal{K}_{i}^{(2)}=\frac{\overline{\mathcal{Z}}_{i}}{\sqrt{\sum_{l=1}^{N} \overline{\mathcal{Z}}_{l}^{2}}} \frac{1}{\sqrt[4]{3}}=\frac{\bar{z}_{i}}{\sqrt{\sum_{l=1}^{N} \bar{z}_{l}^{2}}} \frac{1}{\sqrt[4]{3}} .
$$

Although we have not considered the radial vibrations due to the strong binding of the ions in the radial direction, a detailed treatment of the ion motion in the trap would require the extension to all three dimensions. Then Eq. (3.3) has to be replaced with

$$
\mathbf{q}_{i}=\overline{\mathbf{q}}_{i}+\Delta \mathbf{q}_{i}, \quad i=1, \ldots, N
$$

where $\overline{\mathbf{q}}_{i}$ denotes the equilibrium position of the $i$ th ion in the 3D space and $\Delta \mathbf{q}_{i}$ is its displacement from the equilibrium position. We can write

$$
\begin{aligned}
\overline{\mathbf{q}}_{i} & =\bar{x}_{i} \mathbf{x}+\bar{y}_{i} \mathbf{y}+\bar{z}_{i} \mathbf{z}, \\
\Delta \mathbf{q}_{i} & =\Delta_{i} \mathbf{x}+\Delta_{N+i} \mathbf{y}+\Delta_{2 N+i} \mathbf{z}, \quad i=1,2, \ldots, N,
\end{aligned}
$$

where $\bar{x}_{i}, \bar{y}_{i}, \bar{z}_{i}$ are the equilibrium positions of the $i$ th ion and $\mathbf{x}, \mathbf{y}, \mathbf{z}$ are unit vectors in the 3D space. The free Hamiltonian associated with the vibrational motion in the $3 \mathrm{D}$ space reads

$$
\hat{H}_{e x t}^{(3 D)}=\sum_{\alpha=1}^{3 N} \hbar \nu_{\alpha}\left(\hat{a}_{\alpha}^{\dagger} \hat{a}_{\alpha}+1 / 2\right)
$$

and the displacement operators in Eq. (3.44) are given as follows

$$
\hat{\Delta}_{i}=\sum_{\alpha=1}^{3 N} \mathcal{K}_{i}^{(\alpha)} z_{0}\left(\hat{a}_{\alpha}^{\dagger}+\hat{a}_{\alpha}\right), \quad i=1, \ldots, 3 N,
$$

where the numerical factors $\mathcal{K}_{i}^{(\alpha)}$ in general have to be determined numerically.

\section{LASER-ION INTERACTIONS}

Information is encoded in internal (atomic) states, while it is transferred via external (motional) states of the ions. We can manipulate these states due to laser-ion interactions. It can be accomplished in the travelling-wave and standing-wave configurations. We will address in detail both approaches in what follows. However, we should first comment on the selection of the two internal atomic levels to form the qubit. There are three possibilities [20]:

- We can employ a ground and metastable fine structure excited state. This applies for ions with zero nuclear angular momentum [FIG. 2(a)]. In this case we refer to the single beam scheme and we can drive transitions on optical frequencies. This configuration is used, for example, by the group in Innsbruck using Calcium ions ${ }^{40} \mathrm{Ca}^{+}$[12, 25].

- We can also choose two sublevels of a ground state within the hyperfine structure (ions with nonzero nuclear angular momentum) [FIG.2(b)]. The spacing of such two sublevels is in the range of GHz. Thus, a two-beam Raman scheme via a third virtual level is required in order to resolve the individual sublevels. Experiments in this configuration with Beryllium ions ${ }^{9} \mathrm{Be}^{+}$were performed in Boulder 1, 22, 26. 
- It also possible to apply a magnetic field and consider two Zeeman sublevels of the ground state [FIG.22(c)]. This scheme also requires Raman excitation. In this class, we can mention, for example, Magnesium ions ${ }^{24} \mathrm{Mg}^{+}$ used by the group in Garching [27.

We have to mention also other active groups running experiments towards quantum logic with trapped ions. For instance (in alphabetical order) IBM Almaden using ${ }^{138} \mathrm{Ba}^{+}$28], Imperial College $\left({ }^{40} \mathrm{Ca}^{+},{ }^{199} \mathrm{Hg}^{+}\right)$[29], JPL in Los Angeles $\left({ }^{199} \mathrm{Hg}^{+}\right)$[30], Los Alamos National Laboratory $\left({ }^{40} \mathrm{Ca}^{+}\right)$[31, 36], Oxford University $\left({ }^{40} \mathrm{Ca}^{+}\right)$[19, 32, University of Aarhus $\left({ }^{40} \mathrm{Ca}^{+}\right)$33], University of Hamburg $\left({ }^{138} \mathrm{Ba}^{+},{ }^{171} \mathrm{Yb}^{+}\right)$34 and University of Mainz $\left({ }^{40} \mathrm{Ca}^{+}\right)$ [35.

We can use dipole and quadrupole transitions. Theoretically, the difference is only in the interaction constants as we will see later on in this section. On the other hand, in experiments quadrupole transitions have much longer lifetimes (one second for Calcium ions) comparing to fast decaying dipole transitions $\left(10^{-8} \mathrm{~s}\right)$. Experiments on an octupole transition in an Ytterbium ion has also been realized. The predicted theoretical lifetime in this system is of the order of $10^{8} \mathrm{~s}$ [37]. However, in this case one deals with very weak transitions with very stringent demands on the laser sources used in the experiment (although they are of major interest as potential ion trap clocks). Moreover, weak transitions have to be driven with a very intense laser which enhances the possibility for off-resonant excitations. From now on we will describe in this paper all experimental procedures for Calcium ions ${ }^{40} \mathrm{Ca}^{+}$(FIG. 3) .

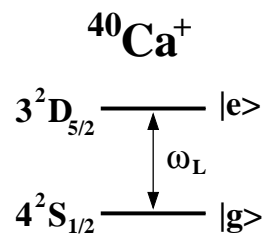

(a)

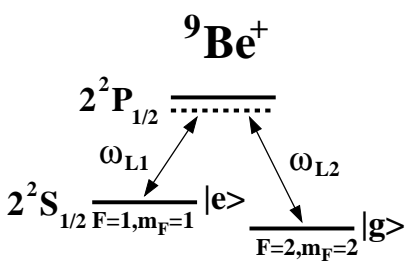

(b)

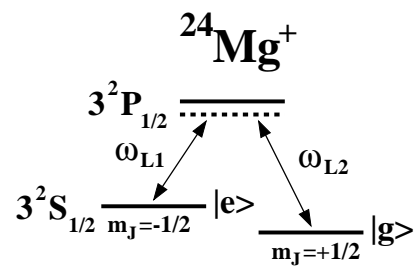

(c)

FIG. 2: Three possible choices for two internal atomic states representing the qubit: (a) a ground and a metastable excited state, (b) sublevels of a ground state and (c) Zeeman sublevels of a ground state, where $\omega_{L}, \omega_{L 1}$ and $\omega_{L 2}$ refer to the laser frequencies 47 .

In the following we will deal with the single beam scheme, i.e. transitions being driven by a single laser beam. We will not treat here the Raman scheme. The derivation of the Hamiltonian in this scheme can be found in Ref. [40. We just mention that the final Hamiltonian in the Raman scheme has the same form as the one in the single beam scheme, except for differences in coupling constants and for atomic frequencies which are Stark light shifted. In the Raman scheme the resulting effective light field has the direction (frequency) determined by the difference of the wavevectors (frequencies) of the two participating laser beams, where each beam is represented (in a semiclassical approach) with a monochromatic travelling wave. Finally, the single beam scheme requires a very high laser frequency stability, while in the Raman scheme we only need to control the relative frequency stability between the two laser beams which is technically less demanding. With the Raman scheme we can also ensure the relative wavevector of the two beams to be parallel to the trap axis which suppresses the coupling to radial motional modes. On the other hand, the Raman scheme can introduce significant Stark light shifts [22].

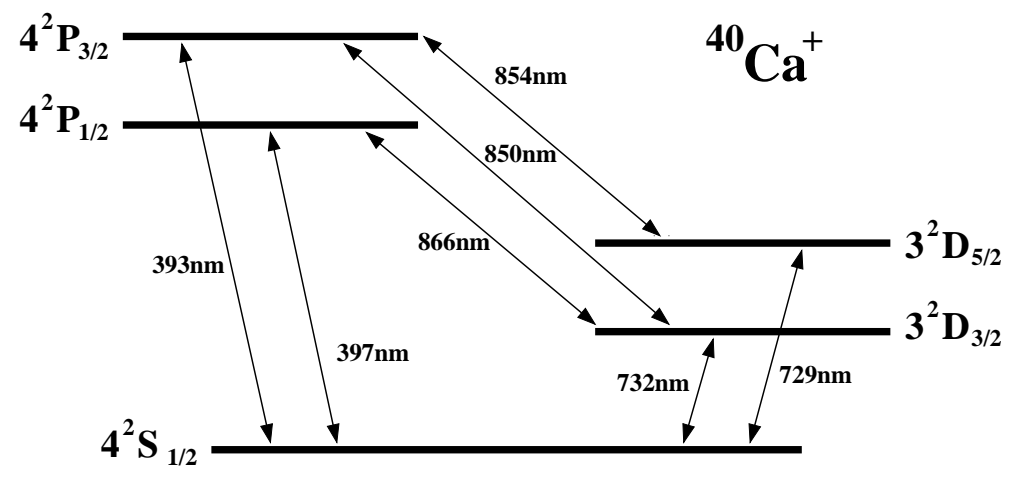

FIG. 3: Five lowest available atomic levels of the Calcium ion ${ }^{40} \mathrm{Ca}^{+} 14$. All transitions are accessible with solid state diode lasers. The nuclear spin of this isotope is zero, i.e. there is no hyperfine structure. For the spectroscopic notation of the levels see the text.

In the rest of the paper we will use the standard atomic level notation $n^{2 S+1} L_{J}$, where $n$ is the principal quantum number, $S$ is the spin angular momentum, $L$ is the orbital angular momentum and $J$ is the total angular momentum 
of electrons. For the fine structure case the notation is $n^{2 S+1} L_{J}\left(m_{J}\right)$ where $m_{J}$ is the projection of $J$ onto the quantization axis. In the case of the hyperfine structure we denote $n^{2 S+1} L_{J}\left(F, m_{F}\right)$ where $F$ is the total angular momentum of the atom (electrons + nucleus) and $m_{F}$ is the projection of $F$ onto the quantization axis.

Let us consider that the ion has two internal levels, denoted $|g\rangle$ (lower) and $|e\rangle$ (upper) with corresponding energies $E_{g}$ and $E_{e}$, where the transition frequency is $\omega_{0}=\left(E_{e}-E_{g}\right) / \hbar$. Then the free Hamiltonian associated with the internal degrees of freedom is given by

$$
\hat{H}_{\text {int }}=E_{e}|e\rangle\left\langle e\left|+E_{g}\right| g\right\rangle\langle g|=\frac{\hbar \omega_{0}}{2} \sigma_{z}+\frac{E_{e}+E_{g}}{2} \mathbb{1}_{\text {int }},
$$

where $\sigma_{z}=|e\rangle\langle e|-| g\rangle\langle g|$ and $\mathbb{1}_{\text {int }}=|e\rangle\langle e|+| g\rangle\langle g|$. Finally, we can write the total free Hamiltonian for the $j$ th ion of $N$ ions confined in the trap communicating via one of the collective vibrational modes [see Eq. (3.36)]

$$
\hat{H}_{0 j}=\hat{H}_{i n t}+\hat{H}_{e x t}=\frac{\hbar \omega_{0}}{2} \sigma_{z j}+\hbar \nu \hat{a}^{\dagger} \hat{a},
$$

where we have omitted constant terms $\left(E_{e}+E_{g}\right) / 2, \hbar \nu / 2$ and dropped down the index $\alpha$ denoting a vibrational mode. The motional mode used for manipulations (especially quantum logic operations) with the ions is called the quantum data bus because, as we will see later, it serves to transfer the information between distinct ions within the ion crystal (representing a quantum register). We will consider for this purpose only the COM mode $\left(\nu=\omega_{z}\right)$ or the breathing mode $\left(\nu=\omega_{z} \sqrt{3}\right)$.

Further, we assume a powerful laser, i.e. the interaction with the ions has no influence on the laser photon statistics. Therefore, we will employ a semiclassical description of the laser beam. We will consider the laser beam in the (i) travelling-wave and (ii) standing-wave configuration.

\section{A. Travelling-wave configuration}

There are two different ways for addressing the ions. We can set the laser beam at a fixed position and shift the ion string by a very slight variation of the DC voltage on the ring electrode. On the other hand, we can fix the ion string and scan the laser across the string. In this case an acousto-optical modulator is used for laser beam deflection [15].

Let us approximate the laser beam as a monochromatic travelling wave (FIG. đ). We can write

$$
\begin{aligned}
\mathbf{E} & =E_{0} \boldsymbol{\epsilon} \cos \left(\omega_{L} t-\boldsymbol{\kappa} \cdot \mathbf{q}+\phi\right), \\
& =\frac{E_{0} \boldsymbol{\epsilon}}{2}\left[e^{-i\left(\omega_{L} t-\boldsymbol{\kappa} \cdot \mathbf{q}+\phi\right)}+e^{i\left(\omega_{L} t-\boldsymbol{\kappa} \cdot \mathbf{q}+\phi\right)}\right],
\end{aligned}
$$

where $E_{0}$ is the real amplitude, $\boldsymbol{\epsilon}$ is the polarization vector with $|\boldsymbol{\epsilon}|=1, \omega_{L}$ is the laser frequency, $\boldsymbol{\kappa}=\kappa \mathbf{n}=\left(\omega_{L} / c\right) \mathbf{n}$ is the wavevector with $|\mathbf{n}|=1, c$ is the speed of light, $\mathbf{q}$ is the position vector and $\phi$ is the phase factor. The full Hamiltonian for the $j$ th ion is given by

$$
\hat{H}_{j}=\hat{H}_{0 j}+\hat{V}_{j},
$$

where the interaction Hamiltonian (assuming a hydrogen-like atomic configuration) expanded to second order (neglecting magnetic dipole interaction) has only two terms

$$
\hat{V}_{j}=\hat{V}_{j}^{D P}+\hat{V}_{j}^{Q D} .
$$

The electric dipole (DP) term is defined as follows

$$
\hat{V}_{j}^{D P}=-q_{e} \sum_{a}\left(\hat{\mathbf{r}}_{j}\right)_{a} E_{a}\left(t, \hat{\mathbf{R}}_{j}\right)=-q_{e} \hat{\mathbf{r}}_{j} \cdot \mathbf{E}\left(t, \hat{\mathbf{R}}_{j}\right),
$$

summing over $a=x, y, z$. We refer to Eq. (4.6) as the dipole approximation. The electric quadrupole (QD) term reads

$$
\hat{V}_{j}^{Q D}=-\frac{q_{e}}{2} \sum_{a, b}\left(\hat{\mathbf{r}}_{j}\right)_{a}\left(\hat{\mathbf{r}}_{j}\right)_{b} \frac{\partial E_{b}\left(t, \hat{\mathbf{R}}_{j}\right)}{\partial q_{a}}
$$

where the sum is applied over $a, b=x, y, z$ and we refer to Eq. (4.7) as the quadrupole approximation. We denote $q_{e}$ to be the electron charge, $\hat{\mathbf{r}}_{j}$ is the internal position operator associated with the position of the valence electron in 


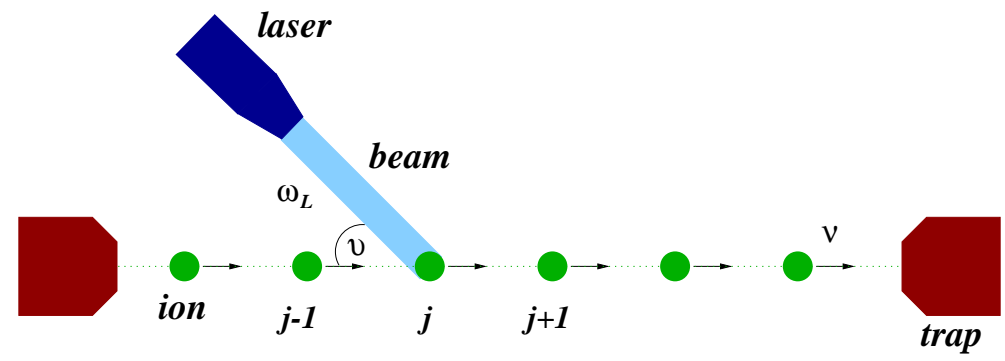

FIG. 4: The travelling-wave configuration corresponds to illuminating the $j$ th ion in the ion string with the laser beam of the frequency $\omega_{L}$ at the angle $\vartheta$ to the trap axis.

the $j$ th ion and $\hat{\mathbf{R}}_{j}=\left(0,0, \hat{z}_{j}\right)$ is the external position operator corresponding to the position of the $j$ th ion in the trap.

For the present we will consider only the dipole term (4.6), regarding to the situation when the dipole interaction is present and the quadrupole contribution (4.7) is then negligible. Later we will also comment on the quadrupole interaction. If we consider consider only a single motional mode, we get from Eq. (3.39) for the external position operator of the $j$ ion

$$
\hat{z}_{j}=\bar{z}_{j}+\mathcal{K}_{j} z_{0}\left(\hat{a}^{\dagger}+\hat{a}\right) .
$$

Then we can sandwich the internal position operator $\hat{\mathbf{r}}_{j}$ with the unity operator $\mathbb{1}_{j}=\left|e_{j}\right\rangle\left\langle e_{j}|+| g_{j}\right\rangle\left\langle g_{j}\right|$ and rewrite Eq. (4.6) to the form

$$
\hat{V}_{j}=-q_{e}\left[\left(\mathbf{r}_{e g}\right)_{j} \hat{\sigma}_{+j}+\left(\mathbf{r}_{e g}\right)_{j}^{*} \hat{\sigma}_{-j}\right] \cdot \frac{E_{0} \boldsymbol{\epsilon}}{2}\left\{e^{-i\left[\omega_{L} t-\eta_{j}\left(\hat{a}^{\dagger}+\hat{a}\right)+\phi_{j}\right]}+\text { H.c. }\right\},
$$

where $\left(\mathbf{r}_{e g}\right)_{j}=\left\langle e_{j}\left|\hat{\mathbf{r}}_{j}\right| g_{j}\right\rangle, \hat{\sigma}_{+j}=\left|e_{j}\right\rangle\left\langle g_{j}\left|, \hat{\sigma}_{-j}=\right| g_{j}\right\rangle\left\langle e_{j}\right|, \kappa=\omega_{L} / c, \eta_{j}=\mathcal{K}_{j} \bar{\eta}, \bar{\eta}=\kappa_{\vartheta} z_{0}, \kappa_{\vartheta}=\kappa \cos \vartheta, \phi_{j}=\phi-\kappa_{\vartheta} \bar{z}_{j}$ with $\mathcal{K}_{j}$ and $z_{0}$ defined by Eq. (3.40). In Eq. (4.9) we consider that $\left\langle e_{j}\left|\hat{\mathbf{r}}_{j}\right| e_{j}\right\rangle=\left\langle g_{j}\left|\hat{\mathbf{r}}_{j}\right| g_{j}\right\rangle=0$, because we assume spatial symmetry of the wavefunctions associated with the internal atomic states $\left|g_{j}\right\rangle$ and $\left|e_{j}\right\rangle$. The schematic configuration is depicted in FIG. A. It is useful to transform to the interaction picture defined by the prescription

$$
\begin{aligned}
& i \hbar \frac{\partial}{\partial t}|\Psi\rangle=\hat{H}|\Psi\rangle \quad \longrightarrow \quad i \hbar \frac{\partial}{\partial t}|\psi\rangle=\hat{\mathcal{H}}|\psi\rangle, \quad|\psi\rangle=\hat{U}_{0}^{\dagger}|\Psi\rangle, \\
& \hat{H}=\hat{H}_{0}+\hat{V} \quad \longrightarrow \quad \hat{\mathcal{H}}=\hat{U}_{0}^{\dagger} \hat{V} \hat{U}_{0}, \quad \hat{U}_{0}=\exp \left(-\frac{i \hat{H}_{0} t}{\hbar}\right) .
\end{aligned}
$$

The Hamiltonian (4.9) after the transformation to the interaction picture (4.10) reads

$$
\hat{\mathcal{H}}_{j}=\frac{\hbar \lambda_{j}}{2} \hat{\sigma}_{+j} \exp \left[i \eta_{j}\left(\hat{a}^{\dagger} e^{i \nu t}+\hat{a} e^{-i \nu t}\right)\right] e^{-i \delta t}+\text { H.c. },
$$

where $\delta=\omega_{L}-\omega_{0}$ and we have neglected rapidly oscillating terms at the frequency $\omega_{L}+\omega_{0}$ compared with lowfrequency terms at $\omega_{L}-\omega_{0}$. In practice $\omega_{L} \simeq \omega_{0}$, therefore to a good degree of approximation for times of interest, high-frequency terms average to zero [21]. This approximation is called the rotating wave approximation ( $R W A)$. In Eq. (4.11) we substitute $\eta_{j}=\bar{\eta} / \sqrt{N}$ for the COM mode or $\eta_{j}=\bar{\eta} \bar{z}_{j} /\left(\sqrt[4]{3} \sum_{l=1}^{N} \bar{z}_{l}^{2}\right)$ for the breathing mode. The laser coupling constant $\lambda_{j}$ introduced in Eq. (4.11) is defined by the relation

$$
\lambda_{j}^{D P}=-\frac{q_{e} E_{0}}{\hbar}\left[\sum_{a}\left\langle e_{j}\left|\left(\hat{\mathbf{r}}_{j}\right)_{a}\right| g_{j}\right\rangle \epsilon_{a}\right] e^{-i \phi_{j}}=-\frac{q_{e} E_{0}}{\hbar}\left[\left(\mathbf{r}_{e g}\right)_{j} \cdot \boldsymbol{\epsilon}\right] e^{-i \phi_{j}} .
$$

However, for a dipole forbidden transition when $\left\langle e_{j}\left|\hat{\mathbf{r}}_{j}\right| g_{j}\right\rangle=0$, the dipole term 4.6$)$ does not contribute $\left(\lambda_{j}^{D P}=0\right)$ and the key role is played by the weaker quadrupole interaction. In that case the laser coupling constant in the Hamiltonian (4.11) reads

$$
\lambda_{j}^{Q D}=-\frac{i q_{e} E_{0} \omega_{L}}{2 \hbar c}\left[\sum_{a, b}\left\langle e_{j}\left|\left(\hat{\mathbf{r}}_{j}\right)_{a}\left(\hat{\mathbf{r}}_{j}\right)_{b}\right| g_{j}\right\rangle n_{a} \epsilon_{b}\right] e^{-i \phi_{j}}
$$


where all parameters are defined in Eq. (4.3).

Next, let us assume the detuning $\delta$ of the laser frequency $\omega_{L}$ from the atomic frequency $\omega_{0}$ for the vibrational frequency $\nu$ in the form

$$
\delta=\omega_{L}-\omega_{0}=k \nu, \quad k=0, \pm 1, \pm 2, \ldots,
$$

and apply the Baker-Campbell-Hausdorff theorem [21] $e^{\hat{A}+\hat{B}}=e^{\hat{A}} e^{\hat{B}} e^{-\frac{1}{2}[\hat{A}, \hat{B}]}$ Eq. (4.11). Then we can write

$$
\hat{\mathcal{H}}_{j}=\frac{\hbar \lambda_{j}}{2} \hat{\sigma}_{+j} e^{-\left(\eta_{j}^{2} / 2\right)} \sum_{\alpha, \beta=0}^{\infty}\left(i \eta_{j}\right)^{\alpha+\beta} \frac{\left(\hat{a}^{\dagger}\right)^{\alpha}}{\alpha !} \frac{\hat{a}^{\beta}}{\beta !} e^{i \nu t(\alpha-\beta-k)}+\text { H.c. } .
$$

If the laser is tuned at the frequency $\omega_{L}$ such that $k>0$, the spectral line is termed the $k$ th blue sideband. For $k=0$ the line is called the carrier and for $k<0$ refers the $k$ th red sideband because the laser is red (blue) detuned from the atomic frequency $\omega_{0}$ (FIG.5).

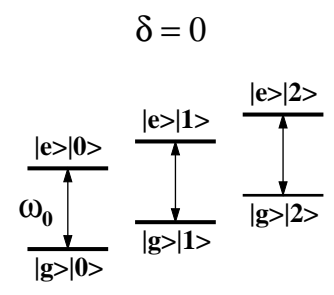

(a)

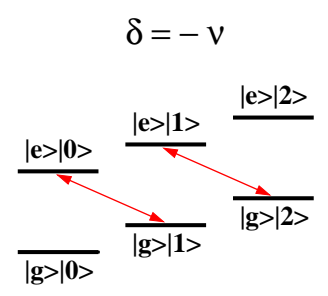

(b)

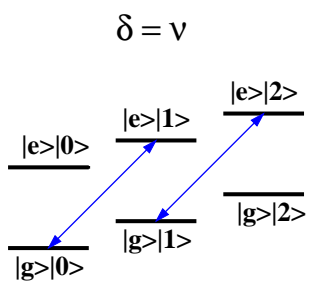

(c)

FIG. 5: Scheme for the transition driven on the carrier (a), on the first red sideband (b) and on the first blue sideband (c), where $\omega_{L}$ denotes the laser frequency, $\omega_{0}$ is the atomic frequency and $\nu$ is the vibrational frequency of the respective motional mode. The parameter $\delta$ is the detuning defined as $\delta=\omega_{L}-\omega_{0}$.

When the constant $\lambda_{j}$ is sufficiently small we can assume that there are no excitations on off-resonant transitions (weak coupling regime). Then the level structure of the ion can be considered as a series of isolated two-level systems 411. Precisely what is meant by sufficiently small is detailed in Appendix A. Assuming the weak coupling regime, we can neglect off-resonant terms $(\alpha-\beta-k \neq 0)$ and rewrite Eq. 4.15) for $k \geq 0$ to the form

$$
\hat{\mathcal{H}}_{j}^{(+)}=\frac{\hbar \lambda_{j}}{2} \hat{\sigma}_{+j}\left(\hat{a}^{\dagger}\right)^{|k|} \mathcal{F}_{k}\left(\hat{a}^{\dagger} \hat{a}\right)+\frac{\hbar \lambda_{j}^{*}}{2} \hat{\sigma}_{-j} \mathcal{F}_{k}^{\dagger}\left(\hat{a}^{\dagger} \hat{a}\right) \hat{a}^{|k|}
$$

and for $k<0$

$$
\hat{\mathcal{H}}_{j}^{(-)}=\frac{\hbar \lambda_{j}}{2} \hat{\sigma}_{+j} \mathcal{F}_{k}\left(\hat{a}^{\dagger} \hat{a}\right) \hat{a}^{|k|}+\frac{\hbar \lambda_{j}^{*}}{2} \hat{\sigma}_{-j}\left(\hat{a}^{\dagger}\right)^{|k|} \mathcal{F}_{k}^{\dagger}\left(\hat{a}^{\dagger} \hat{a}\right) .
$$

In the last relation we introduce the operator function

$$
\mathcal{F}_{k}\left(\hat{a}^{\dagger} \hat{a}\right)=e^{-\left(\eta_{j}^{2} / 2\right)}\left(i \eta_{j}\right)^{|k|} \sum_{\alpha=0}^{\infty}\left(-\eta_{j}^{2}\right)^{\alpha} \frac{\left(\hat{a}^{\dagger} \hat{a}\right)^{\alpha}}{\alpha !(\alpha+|k|) !} .
$$

Although we allow the parameter $k$ to be positive or negative, we keep writing its absolute value $|k|$ in both cases in order to avoid the tricky notation of form $\left(\hat{a}^{\dagger}\right)^{-k}$ and $\hat{a}^{-k}$ in the relation (4.17) and also in what follows next. The final form of the Hamiltonian is given by

$$
\hat{\mathcal{H}}_{j}^{(+)}=\hbar \sum_{n=0}^{\infty}\left[\frac{\Omega_{j}^{n, k}}{2}\left(\left|e_{j}\right\rangle\left\langle g_{j}|\otimes| n+|k|\right\rangle\langle n|\right)+\frac{\left(\Omega_{j}^{n, k}\right)^{*}}{2}\left(\left|g_{j}\right\rangle\left\langle e_{j}|\otimes| n\right\rangle\langle n+|k||\right)\right]
$$

and

$$
\hat{\mathcal{H}}_{j}^{(-)}=\hbar \sum_{n=0}^{\infty}\left[\frac{\Omega_{j}^{n, k}}{2}\left(\left|e_{j}\right\rangle\left\langle g_{j}|\otimes| n\right\rangle\langle n+|k||\right)+\frac{\left(\Omega_{j}^{n, k}\right)^{*}}{2}\left(\left|g_{j}\right\rangle\left\langle e_{j}|\otimes| n+|k|\right\rangle\langle n|\right)\right] .
$$


We have defined a new coupling constant

$$
\Omega_{j}^{n, k}=\lambda_{j} e^{-\left(\eta_{j}^{2} / 2\right)}\left(i \eta_{j}\right)^{|k|} \sqrt{\frac{n !}{(n+|k|) !}} L_{n}^{|k|}\left(\eta_{j}^{2}\right),
$$

where

$$
L_{n}^{a}(x)=\sum_{m=0}^{n}(-1)^{m} \frac{x^{m}}{m !}\left(\begin{array}{c}
n+a \\
n-m
\end{array}\right)
$$

is the generalized Laguerre polynomial and $\left(\begin{array}{c}n+a \\ n-m\end{array}\right)=\frac{(n+a) !}{(n-m) !(a+m) !}$. Finally, we may write the unitary evolution operator for time-independent Hamiltonians (4.19) and (4.20)

$$
\hat{\mathcal{U}}_{j}^{( \pm)}=\exp \left(-\frac{i \hat{\mathcal{H}}_{j}^{( \pm)} t}{\hbar}\right)
$$

which is given for $k \geq 0$ by the formula

$$
\begin{aligned}
\hat{\mathcal{U}}_{j}^{(+)} & =\sum_{n=0}^{\infty} \cos \left(\frac{\left|\Omega_{j}^{n, k}\right| t}{2}\right)\left[\left(\left|e_{j}\right\rangle\left\langle e_{j}|\otimes| n+|k|\right\rangle\langle n+|k||\right)+\left(\left|g_{j}\right\rangle\left\langle g_{j}|\otimes| n\right\rangle\langle n|\right)\right] \\
& -i \sum_{n=0}^{\infty} \sin \left(\frac{\left|\Omega_{j}^{n, k}\right| t}{2}\right)\left[\left(\left|e_{j}\right\rangle\left\langle g_{j}|\otimes| n+|k|\right\rangle\langle n|\right) e^{-i \tilde{\phi}_{j}}+\left(\left|g_{j}\right\rangle\left\langle e_{j}|\otimes| n\right\rangle\langle n+|k||\right) e^{i \tilde{\phi}_{j}}\right] \\
& +\sum_{n=0}^{|k|-1}\left|e_{j}\right\rangle\left\langle e_{j}|\otimes| n\right\rangle\langle n|
\end{aligned}
$$

and for $k<0$

$$
\begin{aligned}
\hat{\mathcal{U}}_{j}^{(-)} & =\sum_{n=0}^{\infty} \cos \left(\frac{\left|\Omega_{j}^{n, k}\right| t}{2}\right)\left[\left(\left|e_{j}\right\rangle\left\langle e_{j}|\otimes| n\right\rangle\langle n|\right)+\left(\left|g_{j}\right\rangle\left\langle g_{j}|\otimes| n+|k|\right\rangle\langle n+|k||\right)\right] \\
& -i \sum_{n=0}^{\infty} \sin \left(\frac{\left|\Omega_{j}^{n, k}\right| t}{2}\right)\left[\left(\left|e_{j}\right\rangle\left\langle g_{j}|\otimes| n\right\rangle\langle n+|k||\right) e^{-i \tilde{\phi}_{j}}+\left(\left|g_{j}\right\rangle\left\langle e_{j}|\otimes| n+|k|\right\rangle\langle n|\right) e^{i \tilde{\phi}_{j}}\right] \\
& +\sum_{n=0}^{|k|-1}\left|g_{j}\right\rangle\left\langle g_{j}|\otimes| n\right\rangle\langle n| .
\end{aligned}
$$

We have denoted $\tilde{\phi}_{j}=\phi_{j}-\frac{\pi}{2}|k|$. For each value of $k$ the phase factor $\tilde{\phi}_{j}$ can be chosen arbitrarily for the first application of $\hat{\mathcal{U}}_{j}^{( \pm)}$. However, once chosen, it must be kept track of if subsequent applications of $\hat{\mathcal{U}}_{j}^{( \pm)}$are performed on the $j$ th ion [22]. The real parameter $\left|\Omega_{j}^{n, k}\right|$ is called the Rabi frequency of the transition $\left|e_{j}\right\rangle|n\rangle \leftrightarrow\left|g_{j}\right\rangle|n+| k|\rangle$ or $\left|e_{j}\right\rangle|n+| k|\rangle \leftrightarrow\left|g_{j}\right\rangle|n\rangle$, respectively. This term comes originally from the field of nuclear magnetic resonance (NMR), where it refers to the periodic flipping of a nuclear spin in the magnetic field. It follows that:

- A $4 \pi$-pulse $\left(\left|\Omega_{j}^{n, k}\right| t=4 \pi\right)$ returns the system back to its initial state. For example

$$
\left|e_{j}\right\rangle|n\rangle \stackrel{4 \pi}{\longrightarrow}\left|e_{j}\right\rangle|n\rangle \text {. }
$$

- A $2 \pi$-pulse $\left(\left|\Omega_{j}^{n, k}\right| t=2 \pi\right)$ changes the sign of the state. For instance

$$
\left|g_{j}\right\rangle|n+| k|\rangle \stackrel{2 \pi}{\longrightarrow}-\left|g_{j}\right\rangle|n+| k|\rangle .
$$

- A $\pi$-pulse $\left(\left|\Omega_{j}^{n, k}\right| t=\pi\right)$ implies that

$$
\left|e_{j}\right\rangle|n+| k|\rangle \stackrel{\pi}{\longrightarrow}\left|g_{j}\right\rangle|n\rangle,
$$

where we set the phase factor $\tilde{\phi}_{j}$ to be zero. Other cases may be easily calculated from the formulas (4.24) and (4.25) given above. 
In what follows we will assume that all motional modes are in the Lamb-Dicke regime characterized by the LambDicke limit (Appendix B). Hence $\eta_{j}$ introduced in Eq. (4.9) is called the Lamb-Dicke parameter. The Lamb-Dicke regime facilitates the ground state cooling (Sec. $\mathrm{VB}$ ) and enables to maintain the contrast of Rabi oscillations on a longer time scale [see Eq. (5.8)]. Then the coupling constant (4.21) simplifies to the form

$$
\Omega_{j}^{n, k} \approx \lambda_{j}\left(i \eta_{j}\right)^{|k|} \sqrt{\frac{(n+|k|) !}{n !}} \frac{1}{|k| !} .
$$

For the purpose of coherent manipulations with internal states of cold trapped ions we will be primarily interested in the interaction on the carrier $(k=0)$ and on the first red sideband $(k=-1)$ which will be used for the construction of a wide class of quantum logic gates. The corresponding unitary evolution operators in the Lamb-Dicke regime for the transition on the carrier $(\hat{\mathcal{A}})$ and on the first red sideband $(\hat{\mathcal{B}})$ may be determined from Eq. (4.24) and (4.25) as follows

$$
\begin{aligned}
\hat{\mathcal{A}}_{j} & =\sum_{n=0}^{\infty} \cos \left(\frac{\left|A_{j}^{n}\right| t}{2}\right)\left[\left(\left|e_{j}\right\rangle\left\langle e_{j}|\otimes| n\right\rangle\langle n|\right)+\left(\left|g_{j}\right\rangle\left\langle g_{j}|\otimes| n\right\rangle\langle n|\right)\right] \\
& -i \sum_{n=0}^{\infty} \sin \left(\frac{\left|A_{j}^{n}\right| t}{2}\right)\left[\left(\left|e_{j}\right\rangle\left\langle g_{j}|\otimes| n\right\rangle\langle n|\right) e^{-i \phi_{j}}+\left(\left|g_{j}\right\rangle\left\langle e_{j}|\otimes| n\right\rangle\langle n|\right) e^{i \phi_{j}}\right]
\end{aligned}
$$

and

$$
\begin{aligned}
\hat{\mathcal{B}}_{j} & =\sum_{n=0}^{\infty} \cos \left(\frac{\left|B_{j}^{n}\right| t}{2}\right)\left[\left(\left|e_{j}\right\rangle\left\langle e_{j}|\otimes| n\right\rangle\langle n|\right)+\left(\left|g_{j}\right\rangle\left\langle g_{j}|\otimes| n+1\right\rangle\langle n+1|\right)\right] \\
& -i \sum_{n=0}^{\infty} \sin \left(\frac{\left|B_{j}^{n}\right| t}{2}\right)\left[\left(\left|e_{j}\right\rangle\left\langle g_{j}|\otimes| n\right\rangle\langle n+1|\right) e^{-i \tilde{\phi}_{j}}+\left(\left|g_{j}\right\rangle\left\langle e_{j}|\otimes| n+1\right\rangle\langle n|\right) e^{i \tilde{\phi}_{j}}\right] \\
& +\left|g_{j}\right\rangle\left\langle g_{j}|\otimes| 0\right\rangle\langle 0| .
\end{aligned}
$$

The respective Rabi frequencies in the Lamb-Dicke limit [Eq. (4.29)] for $k=0$ and $k=-1$ are given by

$$
\begin{aligned}
\left|A_{j}^{n}\right| & =\left|\lambda_{j}\right|, \\
\left|B_{j}^{n}\right| & =\left|\lambda_{j}\right| \eta_{j} \sqrt{n+1} .
\end{aligned}
$$

We could by analogy obtain evolution operators for other sideband transitions.

\section{B. Standing-wave configuration}

As an alternative approach to the laser-ion interactions we could choose a standing light field (FIG.6). One can place a mirror in the setup and let the laser beam reflect from it. The counter propagating waves interfere and create a standing-wave configuration with nodes and antinodes. However, it is experimentally very demanding to place an ion precisely to a node or an antinode. Let us approximate the incident laser beam as a monochromatic travelling wave

$$
\mathbf{E}_{i}=E_{0} \boldsymbol{\epsilon} \cos \left(\omega_{L} t-\kappa \cdot \mathbf{q}+\phi\right)
$$

and the reflected beam as a counter propagating travelling wave

$$
\mathbf{E}_{r}=E_{0} \boldsymbol{\epsilon} \cos \left(\omega_{L} t+\boldsymbol{\kappa} \cdot \mathbf{q}+\phi-\pi\right),
$$

where the reflected wave acquires an additional phase $\pi$ on the reflection at the perfect lossless mirror. Then we can write for the resulting standing wave

$$
\mathbf{E}=\mathbf{E}_{i}+\mathbf{E}_{r}=2 E_{0} \boldsymbol{\epsilon} \sin \left(\omega_{L} t+\phi\right) \sin (\boldsymbol{\kappa} \cdot \mathbf{q}),
$$

where the notation is adopted from Eq. (4.3). Following Eq. 44.6) and (4.7) we can write the corresponding relations for the standing wave in the semiclassical representation

$$
\mathbf{E}\left(t, \hat{\mathbf{R}}_{j}\right)=-i E_{0} \boldsymbol{\epsilon}\left[e^{i\left(\omega_{L} t+\phi\right)}-e^{-i\left(\omega_{L} t+\phi\right)}\right] \sin \left[\chi_{j}+\eta_{j}\left(\hat{a}^{\dagger}+\hat{a}\right)\right],
$$


and

$$
\frac{\partial E_{b}\left(t, \hat{\mathbf{R}}_{j}\right)}{\partial q_{a}}=-i E_{0} \kappa_{a} \epsilon_{b}\left[e^{i\left(\omega_{L} t+\phi\right)}-e^{-i\left(\omega_{L} t+\phi\right)}\right] \cos \left[\chi_{j}+\eta_{j}\left(\hat{a}^{\dagger}+\hat{a}\right)\right]
$$

where the new parameter $\chi_{j}=\kappa_{\vartheta} \bar{z}_{j}$ determines the position of the $j$ th ion in the standing wave and $\kappa_{a}=\left(\omega_{L} / c\right) n_{a}$. The notation is adopted from Eq. (4.3) and (4.9). The condition $\chi_{j}=0$ refers to the $j$ th ion placed in the node, whereas $\chi_{j}=\pi / 2$ refers to the ion positioned in the antinode of the standing wave.

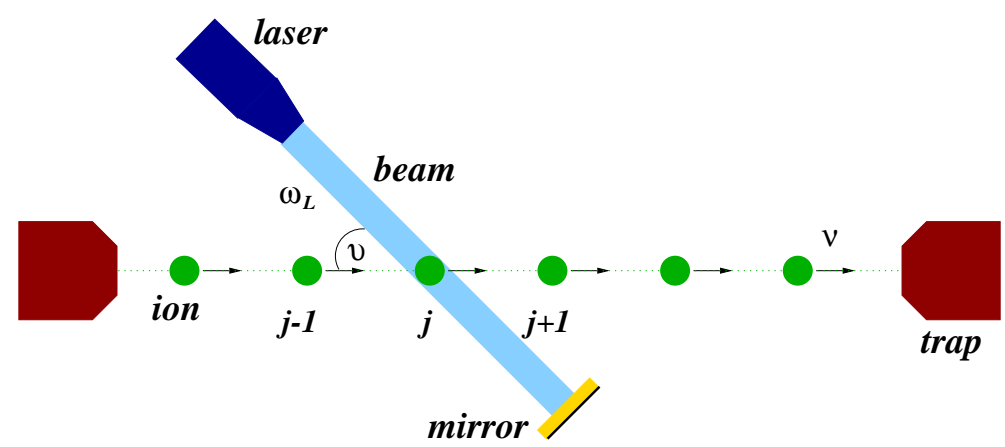

FIG. 6: The standing-wave configuration corresponds to illuminating the $j$ th ion in the ion crystal with the laser beam of the frequency $\omega_{L}$ at the angle $\vartheta$ to the trap axis.

Following the derivation for the travelling-wave configuration, one can easily derive the Hamiltonian in the interaction picture for the standing-wave configuration. It takes the form of the expressions (4.16) and (4.17), except that the laser coupling constant $\lambda_{j}$ and the operator function $\mathcal{F}_{k}$ are replaced with $\tilde{\lambda}_{j}$ and $\tilde{\mathcal{F}}_{k}$. They are given in the dipole approximation (assuming a dipole allowed transition) by

$$
\begin{aligned}
\tilde{\lambda}_{j}^{D P} & =-\frac{i 2 q_{e} E_{0}}{\hbar}\left[\left(\mathbf{r}_{e g}\right)_{j} \cdot \boldsymbol{\epsilon}\right] e^{-i \phi} \\
\tilde{\mathcal{F}}_{k}^{D P}\left(\hat{a}^{\dagger} \hat{a}\right) & =e^{-\left(\eta_{j}^{2} / 2\right)} \sin \left(\chi_{j}+\frac{\pi}{2}|k|\right) \eta_{j}^{|k|} \sum_{\alpha=0}^{\infty}\left(-\eta_{j}^{2}\right)^{\alpha} \frac{\left(\hat{a}^{\dagger} \hat{a}\right)^{\alpha}}{\alpha !(\alpha+|k|) !}
\end{aligned}
$$

and in the quadrupole approximation (assuming a dipole forbidden transition) by

$$
\begin{aligned}
\tilde{\lambda}_{j}^{Q D} & =-\frac{i q_{e} E_{0} \omega_{L}}{\hbar c}\left[\sum_{a, b}\left\langle e_{j}\left|\left(\hat{\mathbf{r}}_{j}\right)_{a}\left(\hat{\mathbf{r}}_{j}\right)_{b}\right| g_{j}\right\rangle n_{a} \epsilon_{b}\right] e^{-i \phi} \\
\tilde{\mathcal{F}}_{k}^{Q D}\left(\hat{a}^{\dagger} \hat{a}\right) & =e^{-\left(\eta_{j}^{2} / 2\right)} \cos \left(\chi_{j}+\frac{\pi}{2}|k|\right) \eta_{j}^{|k|} \sum_{\alpha=0}^{\infty}\left(-\eta_{j}^{2}\right)^{\alpha} \frac{\left(\hat{a}^{\dagger} \hat{a}\right)^{\alpha}}{\alpha !(\alpha+|k|) !} .
\end{aligned}
$$

Comparing the expressions for the coupling constant in the travelling-wave configuration [Eq. (4.12) and (4.13)] with those ones for the standing-wave configuration [Eq. 4.39) and (4.41)], we find out that $\left|\tilde{\lambda}_{j}^{D P}\right|=2\left|\lambda_{j}^{D P}\right|$ and $\left|\tilde{\lambda}_{j}^{Q D}\right|=2\left|\lambda_{j}^{Q D}\right|$. The factor 2 arises from the expression of the standing wave (4.36) where we have superposed two travelling waves with equal amplitudes. Finally, the Hamiltonian can be written in the form given by Eq. (4.19) and (4.20) with the coupling constant in the dipole approximation

$$
\left(\tilde{\Omega}_{j}^{n, k}\right)^{D P}=\lambda_{j}^{D P} e^{-\left(\eta_{j}^{2} / 2\right)} \sin \left(\chi_{j}+\frac{\pi}{2}|k|\right) \eta_{j}^{|k|} \sqrt{\frac{n !}{(n+|k|) !}} L_{n}^{|k|}\left(\eta_{j}^{2}\right)
$$

and in the quadrupole approximation

$$
\left(\tilde{\Omega}_{j}^{n, k}\right)^{Q D}=\lambda_{j}^{Q D} e^{-\left(\eta_{j}^{2} / 2\right)} \cos \left(\chi_{j}+\frac{\pi}{2}|k|\right) \eta_{j}^{|k|} \sqrt{\frac{n !}{(n+|k|) !}} L_{n}^{|k|}\left(\eta_{j}^{2}\right) .
$$

It follows from Eq. (4.43) that for the $j$ th ion in the dipole approximation placed in the node of the standing wave $\left(\chi_{j}=0\right)$ only transitions on odd sidebands $(|k|=2 p+1)$ are present. For the same ion in the antinode $\left(\chi_{j}=\pi / 2\right)$ 
only even sidebands $(|k|=2 p)$ are present, where $p$ is an integer or the zero. In the quadrupole approximation the statements above are valid in the opposite order [compare Eq. (4.43) with (4.44)]. The reason for missing transitions in the standing-wave configuration comes from the destructive interference between the two counter propagating travelling waves in the standing-wave field.

We could easily write the coupling constant $\tilde{\Omega}_{j}^{n, k}$ in the Lamb-Dicke limit [see Eq. (4.29)]. We could also write the unitary evolution operator for the standing-wave configuration. However, it differs from the evolution operator in the travelling-wave configuration [Eq. (4.24) and (4.25)] only in the coupling constant and in the phase factor, but it produces no fundamental problem for further applications. Therefore, in what follows we will consider the expressions and formulas for the travelling-wave configuration keeping in mind the way how to convert to a standingwave configuration.

\section{LASER COOLING}

Laser cooling is the process in which the kinetic energy of atoms is reduced through the action of one or more laser beams. The last decade brought rapid progress in this research field and this effort culminated in 1997 with the award of the Nobel Prize in physics for laser cooling and trapping of atoms 42, 43, 44]. A recent review of different experimental techniques for laser cooling can be found in Ref. [45].

One of the requirements for the practical implementation of quantum computing is the ability to prepare well defined initial states of the qubits [5]. In our case the qubits are represented by trapped ions with vibrational (external) and atomic (internal) degrees of freedom. Laser cooling enables the preparation of well defined initial states of motion and electron shelving serves for the proper initialization of the ion register. We will describe this method later on in Sec. VI. Laser cooling of trapped ions with the axial trapping frequency $\omega_{z}$ has two stages depending on the linewidth $\Gamma$ of the cooling transition [13, 14]:

- Doppler cooling is applied when the vibrational frequency of the ions is smaller than the linewidth of a transition used for cooling $\left(\omega_{z} \leq \Gamma\right)$. In other words, this means that the velocity of the ion due to the trapping potential changes on a longer time scale than the time it takes the ion to absorb or emit a photon (strong laser driving is assumed). Therefore, we can assume that these processes change the momentum of the ion instantaneously. For $\omega_{z} \leq \Gamma$ we refer to the weak confinement regime (in the sense of weak binding of the ions to the ion trap).

- Sideband cooling is used for further cooling below the Doppler cooling limit and requires the vibrational frequency to be much bigger than the linewidth $\left(\omega_{z} \gg \Gamma\right)$. Under this condition the ion develops well resolved sidebands and cooling to a lowest vibrational state is realized through driving a lower sideband. For $\omega_{z} \gg \Gamma$ we refer to the strong confinement regime. One can use instead a novel technique called laser cooling using electromagnetically induced transparency.

\section{A. Doppler cooling}

This stage of laser cooling is based on the Doppler effect. The technique is based on the fact that moving atoms absorb photons from a counter propagating red detuned laser beam (tuned slightly below the atomic frequency) and emit spontaneously in a random direction. After several such cooling cycles (absorption followed by spontaneous emission) we can write for the total momentum $\mathbf{p}$ of atoms

$$
\mathbf{p}=\mathbf{p}_{0}+\sum_{j} \hbar \mathbf{k}_{j}^{(a b s)}+\sum_{j} \hbar \mathbf{k}_{j}^{(e m)}
$$

where $\mathbf{p}_{0}$ is the initial momentum of atoms, $\mathbf{k}_{j}^{(a b s)}$ and $\mathbf{k}_{j}^{(e m)}$ denotes the wavevectors of the absorbed and emitted photons in the $j$ th cooling cycle. We usually use a fast decaying dipole transition for the Doppler cooling, therefore the spontaneous emission is much faster than stimulated emission. The average total momentum of atoms after many cooling cycles takes the form

$$
\langle\mathbf{p}\rangle=\mathbf{p}_{0}+\hbar \mathbf{k}_{L}\langle n\rangle,
$$

where $\langle n\rangle$ is the average number of absorption and emission events (typically $\langle n\rangle \simeq 10^{3}-10^{4}$ ) and by the definition $\mathbf{k}_{L}=\mathbf{k}_{j}^{(a b s)}\left(\mathbf{k}_{L}\right.$ is the wavevector associated with the laser light). The spontaneous contribution averages to zero because it is randomly distributed over the solid angle $4 \pi$. If the laser is red detuned and counter propagating to the 
motion of atoms $\left(\mathbf{p}_{0} \uparrow \downarrow \mathbf{k}_{L}\right)$, then the velocity of the atoms is significantly decreased [see Eq. (5.2)]. For more details see ref. 11, 15.

The discussion above is valid for free atoms but it also applies for trapped ions [46], where the motion towards the laser is provided by the periodic vibrations. The Doppler cooling limit corresponds to the final temperature $T_{\text {dopp }}=\hbar \Gamma / 2 k$, where $\Gamma$ is the linewidth of the cooling transition and $k$ is the Boltzmann constant. This temperature is typically of the order of $\mathrm{mK}$ [47]. However, the Doppler cooling limit can be also translated into the minimum average phonon number in the axial direction [11]

$$
\left\langle n_{z}\right\rangle_{\min }=\frac{\Gamma}{\omega_{z}}\left(\frac{1+\alpha}{4}\right)\left(\frac{\Gamma}{\delta}+\frac{\delta}{\Gamma}\right)-\frac{1}{2}
$$

where $\Gamma$ is the natural linewidth of the cooling transition, $\alpha$ is determined from the angular distribution of the emitted radiation and $\delta$ is the laser detuning from the atomic frequency. For a dipole radiation pattern we get $\alpha=2 / 5$. The cooling is optimal for the detuning $\delta=\Gamma \gg \omega_{z}$. Concerning this condition we can rewrite Eq. (5.3) for a dipole transition to the form

$$
\left\langle n_{z}\right\rangle_{\min } \simeq \frac{7}{10} \frac{\Gamma}{\omega_{z}}
$$

We have omitted the factor $1 / 2$ corresponding to the zero point energy because it has a negligible contribution. The Doppler cooling limit is associated with the recoil of the atoms at the spontaneous emission.

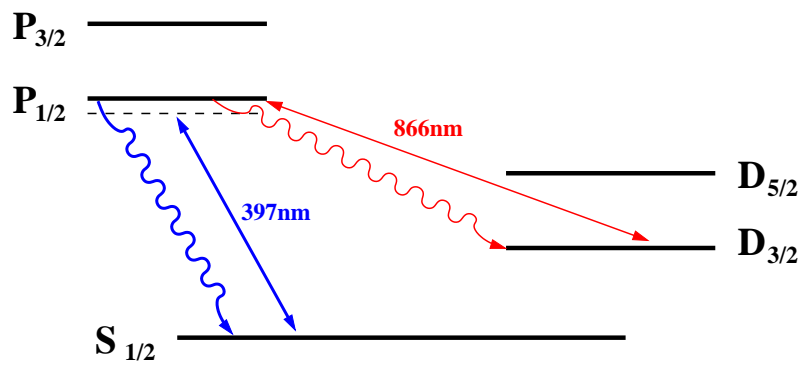

FIG. 7: Doppler cooling. The $S_{1 / 2} \leftrightarrow P_{1 / 2}$ is the cooling transition. The cooling laser on $397 \mathrm{~nm}$ is red detuned by $10 \mathrm{MHz}$. The ion spontaneously decays from the $P_{1 / 2}$ level. There is a $6 \%$ probability of the decay to the metastable state $D_{3 / 2}$, therefore the pumping laser on $866 \mathrm{~nm}$ is switched on.

The relation (5.4) leads us to a discussion of how to choose the axial trapping frequency $\omega_{z}$. In order to be able to address individually each ion with a single laser beam, the minimum spacing between the ions [see Eq. (3.9)] has to be large enough, which requires small $\omega_{z}$. On the other hand, we do not want the frequency $\omega_{z}$ to be too small in order to make the result of Doppler cooling as efficient as possible [Eq. (5.4)]. Thus, the design of ion traps is also determined by the trade-off between these two options.

For Calcium ions ${ }^{40} \mathrm{Ca}^{+}$the $S_{1 / 2} \leftrightarrow P_{1 / 2}$ transition with the natural linewidth $\Gamma=20 \mathrm{MHz}$ is used for the Doppler cooling. The lifetime of the $P_{1 / 2}$ level is about $7 \mathrm{~ns}$ and this level decays with $6 \%$ probability to the metastable $D_{3 / 2}$ level (FIG. [7). Therefore, optical pumping between the $P_{1 / 2}$ and $D_{3 / 2}$ levels is present on $866 \mathrm{~nm}$. The laser on the cooling transition $S_{1 / 2} \leftrightarrow P_{1 / 2}$ is red detuned by $\Gamma / 2 \simeq 10 \mathrm{MHz}$. On the other hand, the pumping laser at $866 \mathrm{~nm}$ is kept on the resonance in order to prevent population trapping in the superposition of the $S_{1 / 2}$ and $D_{3 / 2}$ levels [14]. For $\alpha=2.5, \omega_{z} / 2 \pi \simeq 700 \mathrm{kHz}$ and $\delta=\Gamma / 2$ we can calculate from Eq. (5.3) the minimum average phonon number to be $\left\langle n_{z}\right\rangle_{\min } \simeq 3.5$. This number differs from experimentally measured values (which are bigger) because Eq. (5.3) has been derived for a two-level system while the experimental realization of Doppler cooling involves a three-level system. Nevertheless, $\left\langle n_{z}\right\rangle_{\min } \simeq 3.5$ is still not $\left\langle n_{z}\right\rangle_{\min }=0$ required for proper operation of the quantum processor with cold trapped ions. Therefore a second cooling stage must be launched.

\section{B. Sideband cooling}

Doppler cooling represents the precooling stage in experiments with trapped ions. The final stage can be realized by the sideband cooling technique which may prepare the ions to the ground motional state, i.e. a well defined initial quantum state. Firstly, we address the basic idea of sideband cooling. Then we illustrate this cooling technique on two trapped ions in and outside the Lamb-Dicke regime. Finally, we describe how sideband cooling is realized experimentally. 
In the strong confinement regime $\left(\omega_{z} \gg \Gamma\right)$ a single trapped ion exhibits in its absorption spectrum well resolved sidebands at $\omega_{0} \pm k \omega_{z}$ ( $k$ is an integer) spaced on both sides of the carrier on the atomic frequency $\omega_{0}$. Sideband cooling occurs when the cooling laser is tuned to a lower sideband at $\omega_{L}=\omega_{0}-k \omega_{z}$. In the Lamb-Dicke limit cooling works efficiently with the laser tuned on the first red sideband at $\omega_{L}=\omega_{0}-\omega_{z}$. Then the ion absorbs photons of the energy $\hbar\left(\omega_{0}-\omega_{z}\right)$ and spontaneously emitted photons of the average energy $\hbar \omega_{0}-E_{r}$ bring the ion back to its initial internal state (see Appendix B). In every cooling cycle (absorption + emission) the motional energy of the ion is damped by one vibrational quantum if $\hbar \omega_{z} \gg E_{r}$. This condition implies that in this form the sideband cooling requires the ion to be in the Lamb-Dicke limit. The whole process consists of cooling cycles in which the absorption is followed by the spontaneous emission until the ion reaches the ground motional state $|n=0\rangle$ and decouples from the cooling laser. For the sideband cooling of the single trapped ion initially in the internal state $|g\rangle$ and in the motional state $|n\rangle$, where $a$ denotes the absorption and $e$ stays for the spontaneous emission, we may schematically write

$$
|g\rangle|n\rangle \stackrel{a}{\rightarrow}|e\rangle|n-1\rangle \stackrel{e}{\rightarrow}|g\rangle|n-1\rangle \stackrel{a}{\rightarrow} \ldots \stackrel{e}{\rightarrow}|g\rangle|1\rangle \stackrel{a}{\rightarrow}|e\rangle|0\rangle \stackrel{e}{\rightarrow}|g\rangle|0\rangle .
$$

The minimum average phonon number in the axial direction that can be reached by sideband cooling is then given by $[11$

$$
\left\langle n_{z}\right\rangle_{\min }=\left(\frac{\Gamma}{\omega_{z}}\right)^{2}\left(\alpha+\frac{1}{2}\right),
$$

where the parameter $\alpha$ has been defined in Eq. (5.1). It is evident that now one can achieve efficient cooling to the ground motional state, i.e. $\left\langle n_{z}\right\rangle_{\min } \simeq 0$, assuming the strong confinement regime $\left(\omega_{z} \gg \Gamma\right)$. The limit for the sideband cooling [Eq. (5.5)] is constrained by the recoil of the ion and is determined by the equilibrium between cooling and heating processes. Heating is caused mainly by off-resonant excitations on the carrier $(|g\rangle|n\rangle \leftrightarrow|e\rangle|n\rangle)$ and on the first blue sideband $(|g\rangle|n\rangle \leftrightarrow|e\rangle|n+1\rangle)$. The sideband cooling of a single ion beyond the Lamb-Dicke limit also exists and is based on the creation of a dark state in the energy level structure 48]. A single trapped Mercury ${ }^{198} \mathrm{Hg}^{+}$ion was firstly cooled to the ground motional state in 1989 in Boulder [49], while sideband cooling of a single Beryllium ion ${ }^{9} \mathrm{Be}^{+}$in all three dimensions was firstly reported in 1995 also by the group in Boulder 51.

\section{Sideband cooling of two ions}

The key difference between one and more ions lies in the energy spectrum [19, 20]. While single ions have discrete energy levels (three motional degrees of freedom), a chain of $N$ oscillating ions ( $3 N$ motional degrees of freedom) exhibits a quasicontinuous energy spectrum due to the incommensurate frequencies of the motional modes. For instance, in the axial direction we have the frequencies $\omega_{z}, \omega_{z} \sqrt{3}, \omega_{z} \sqrt{5.8}, \omega_{z} \sqrt{9.3}$, etc.
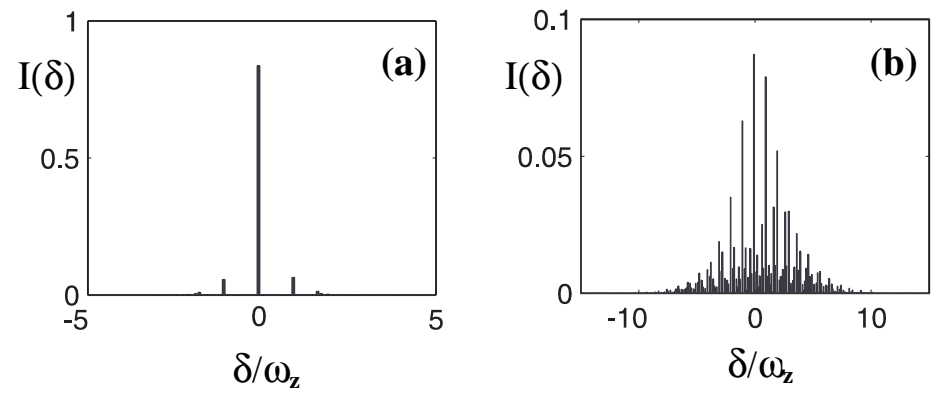

FIG. 8: Absorption spectrum $I(\delta)$ of two ions for a thermal distribution $P(\mathbf{n})$ in the Lamb-Dicke regime (a) and outside the Lamb-Dicke regime (b) as a function of detuning $\delta$ (in units of the axial vibrational frequency $\omega_{z}$ ) where $I(\delta)$ is defined in Eq. (5.6) and $\delta$ in Eq. (4.14). Used by kind permission of Giovanna Morigi and Jürgen Eschner 54].

We will discuss in detail the case of two trapped ions to illustrate the situation of sideband cooling of more than a single ion [54]. The state of each ion $(j=1,2)$ will be expressed in the basis $\left\{\left|g_{j}\right\rangle|\mathbf{n}\rangle,\left|e_{j}\right\rangle|\mathbf{n}\rangle\right\}$, where $\mathbf{n}=\left(n_{1}, n_{2}\right), n_{1}$ is the vibrational number associated with the COM mode $\left(\nu_{1}=\omega_{z}\right)$ and $n_{2}$ with the breathing mode $\left(\nu_{2}=\omega_{z} \sqrt{3}\right)$. The absorption spectrum of the $j$ th ion will be considered in the form 55]

$$
I_{j}(\delta)=\sum_{E_{\mathbf{n}}-E_{\mathbf{m}}=\delta}\left|\left\langle\mathbf{n}\left|\exp \left(i \Delta_{j} \kappa_{\vartheta}\right)\right| \mathbf{m}\right\rangle\right|^{2} P(\mathbf{n}),
$$


where $E_{\mathbf{n}}=\hbar \omega_{z} n_{1}+\hbar \omega_{z} \sqrt{3} n_{2}, \delta$ is the detuning [Eq. (4.14)], $\kappa_{\vartheta}$ is defined by Eq. (4.9) and $\Delta_{j}$ is the displacement operator of the $j$ th ion $[\mathrm{Eq} .(3.39)] . P(\mathbf{n})=P\left(n_{1}, n_{2}\right)$ is a probability distribution associated with the vibrational motion of the ions. The Lamb-Dicke parameter distinguishes between two very different regimes of sideband cooling of more ions:

- In the Lamb-Dicke regime (Appendix B) and in the strong confinement regime $\left(\omega_{z} \gg \Gamma\right)$ only the first sidebands of the motional modes at $\omega_{0} \pm \omega_{z}$ and $\omega_{0} \pm \omega_{z} \sqrt{3}$ appear around the significant carrier peak at $\omega_{0}$ in the absorption spectrum [FIG.8(a)]. The higher sidebands are suppressed due to their strength being proportional to higher powers in the Lamb-Dicke parameter than $\eta$ denoted as $\mathcal{O}\left(\eta^{2}\right)$. Tuning the laser on the first red sideband of the COM mode $\left(\delta=-\omega_{z}\right)$ we can reach its ground state $\left|n_{1}=0\right\rangle$ at the same cooling rate as for a single ion [54. However, in the case of two ions the breathing mode is decoupled from the COM mode and its cooling is almost frozen. Simultaneous cooling of more modes requires the modes to be coupled to the cooling laser and then the requirement of the strong coupling regime $\left(\omega_{z} \gg \Gamma\right)$ has to be reconsidered or one has to use alternative techniques.

- Outside the Lamb-Dicke regime higher sidebands with the strength proportional to $\mathcal{O}\left(\eta^{2}\right)$ also contribute and the absorption spectrum exhibits the structure with many overlapping sidebands at $\omega_{0} \pm k \omega_{z} \pm l \omega_{z} \sqrt{3}$ where $k, l$ are integers [FIG.8(b)]. In this situation the laser tuned on a lower sideband (it does not have to be strictly the first red sideband of the COM mode) excites simultaneously all sideband transitions around this lower sideband in the interval of the linewidth $\Gamma$. Then the COM and the breathing mode are coupled and cooled at once. However, the cooling process is much slower in comparison to cooling of a single ion beyond the Lamb-Dicke limit. It is partly caused by (i) the increasement of the number of the motional modes but also by (ii) the appearance of dark states [54. The dark states are almost decoupled from a resonantly excited state because their motional wave function after the absorption overlaps with the motional wavefunction of the excited state only a little. Thus, the ions may be trapped in these dark states and it slows the cooling process down. This problem can be solved by escaping from the strong confinement regime $\left(\omega_{z} \gg \Gamma\right)$, i.e. by increasing the linewidth $\Gamma$. It will cause that a single level will be coupled to more levels (more sidebands are in the resonance) and the ions will be cooled more efficiently due to more cooling channels. As a result the dark states will disappear because more channels provide more ways for the ion to escape from dark (population trapping) states. Moreover, the rate of the cooling cycles (absorption + emission) is proportional to the linewidth $\Gamma$. Summarizing both effects we can conclude that the total cooling time beyond the Lamb-Dicke limit can be shortened significantly for $\Gamma \simeq \omega_{z}$.

\section{Experimental sideband cooling}

Two ions were cooled for the first time to the ground motional state in 1998 in Boulder. It was achieved on Beryllium ions ${ }^{9} \mathrm{Be}^{+}$illuminating both ions at once [50]. However, it is sufficient to illuminate only one ion from the entire ion string because other ions are cooled sympathetically due to the strong Coulomb coupling. Although we need only one motional mode (COM or breathing axial mode) as the quantum data bus, which has to be in the ground motional state, we require also other modes to be cooled close to the ground state. Uncooled motional modes with thermal phonon distributions significantly affect the Rabi frequency in the data mode and spoil the fidelity of the coherent state manipulation [see Eq. (5.8)].

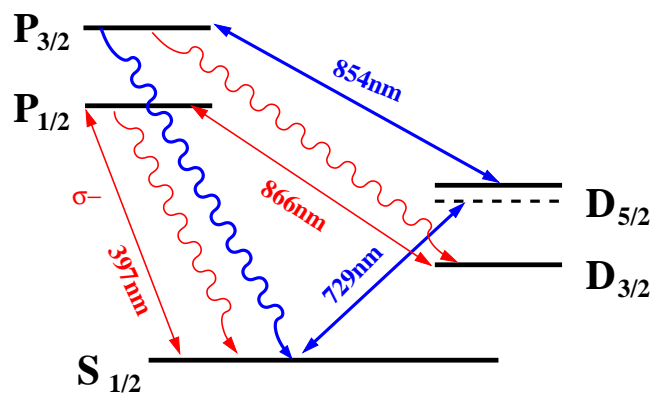

FIG. 9: Sideband cooling with cooling and pumping transitions. The blue transitions correspond to the cooling cycle. The laser at $729 \mathrm{~nm}$ is tuned on the first red sideband. The laser at $854 \mathrm{~nm}$ couples the metastable $D_{5 / 2}$ level with the fast decaying $P_{3 / 2}$ level. The cooling cycle is closed by the spontaneous emission on the fast $S_{1 / 2} \leftrightarrow P_{3 / 2}$ dipole transition. The $P_{3 / 2}$ level may decay to the state $D_{3 / 2}$. The ion is recycled to the state $P_{1 / 2}$ by the laser at $866 \mathrm{~nm}$ followed by the spontaneous emission back to the state $S_{1 / 2}$. Decay to the sublevels $S_{1 / 2}(m=+1 / 2)$ is counteracted by driving the $S_{1 / 2} \leftrightarrow P_{1 / 2}$ transition with the $\sigma^{-}$polarized laser at $397 \mathrm{~nm}$. 
The group in Innsbruck has realized different approaches in sideband cooling of two Calcium ions [16, 17]. If they cool only one motional mode, while the other modes are left in the thermal states, they achieve the ground state population greater than $95 \%(\langle n\rangle \simeq 0.05)$ in the respective mode. However, they can cool sequentially all motional modes close to the ground state. For this purpose they use a small modification in the sideband cooling scheme. The laser frequency and laser power has to be set sequentially for the respective first red sideband of the given motional mode. After sequential cooling all modes the corresponding average phonon numbers are from $\langle n\rangle \simeq 0.05$ to $\langle n\rangle \simeq 2.3$ because the recoil energy from the spontaneous emission in the cooling process of one motional mode reheat other modes.

For Calcium ions ${ }^{40} \mathrm{Ca}^{+}$the quadrupole transition between the two Zeeman sublevels $S_{1 / 2}\left(m_{J}=-1 / 2\right)$ and $D_{5 / 2}\left(m_{J}=-5 / 2\right)$ is used for the sideband cooling (FIG.9). The laser on $729 \mathrm{~nm}$ is tuned on the first red sideband of the respective motional mode and a weak magnetic field is applied for Zeeman splitting of energy levels. The lifetime of the metastable $D_{5 / 2}$ level is about a second, therefore there is the pumping on $854 \mathrm{~nm}$ to the fast decaying $P_{3 / 2}\left(m_{J}=-3 / 2\right)$ level in order to decrease the duration of one cooling cycle, i.e. to increase the cooling rate. The $P_{3 / 2}\left(m_{J}=-3 / 2\right)$ level decays spontaneously to the initial state $S_{1 / 2}\left(m_{J}=-1 / 2\right)$ and closes the cooling cycle. However, the $P_{3 / 2}$ level may decay with a small probability to the $D_{3 / 2}$ level, therefore the pumping laser on $866 \mathrm{~nm}$ recycles the population to the $P_{1 / 2}$ state which decays to the $S_{1 / 2}$ state. The $S_{1 / 2} \leftrightarrow P_{1 / 2}$ transition is driven with the $\sigma^{-}$polarized laser on $397 \mathrm{~nm}$ to counteract the population of the $S_{1 / 2}\left(m_{J}=+1 / 2\right)$ level 14 .

\section{Sympathetic cooling}

In the previous section we have mentioned that it is sufficient to illuminate with cooling lasers only one ion from the ion string because the other ions are cooled sympathetically due to the Coulomb interaction between them. Hence the term sympathetic cooling. However, instead of identical ions one can consider different atomic species (eventually isotopes) in the ion crystal [57]. Then the addressing of cooling ions avoids the disturbance of internal states of logic ions which store the information [58].
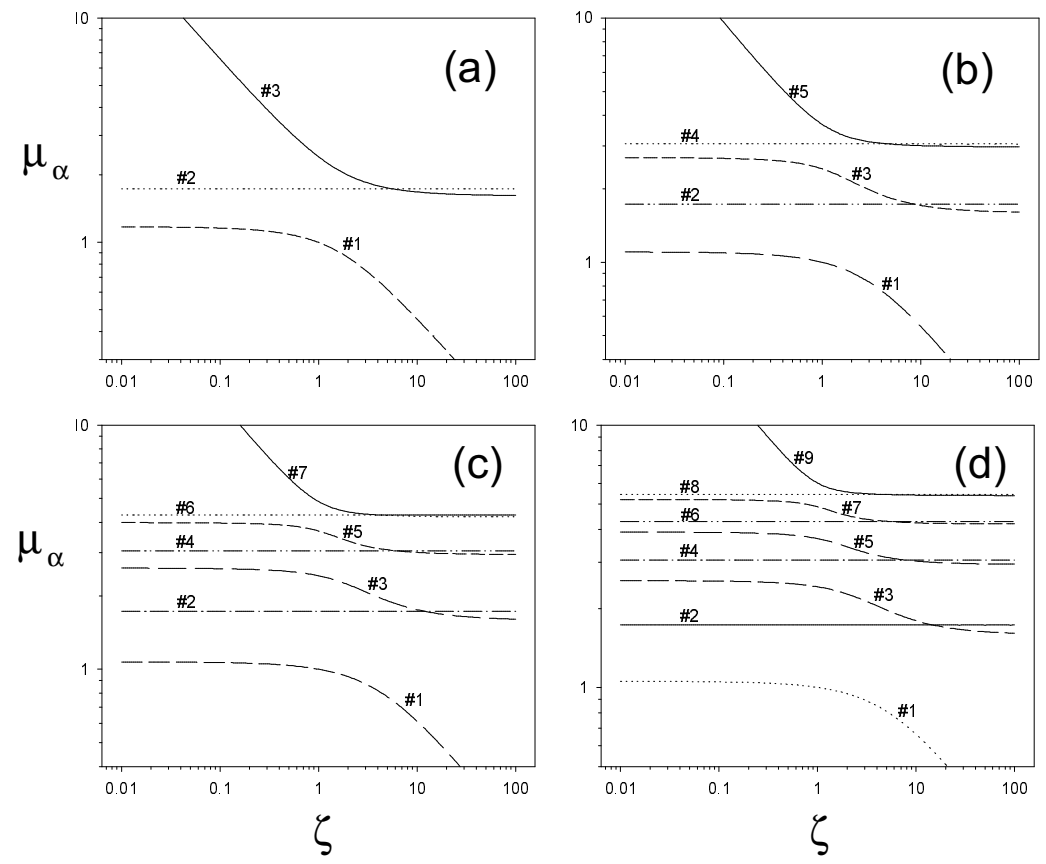

FIG. 10: Normalized axial frequencies 3.28 as a function of $\zeta$ for (a) three, (b) five, (c) seven and (d) nine ions. Used by kind permission of David Wineland and David Kielpinski [59.

Electric fields from the trap electrodes are one of the sources of the motional decoherence of the ion crystal due to heating of collective vibrational motional modes (normal modes). If one assumes the dimension of the ion trap to be much larger than the dimension of the ion crystal, then we can expect the electrode electric fields to be nearly uniform across the ion crystal. Such uniform fields influence and heat only collective motional modes involving the centre-of-mass (COM) motion of the ion crystal. Uniform electric fields can directly heat up the normal mode used 
for quantum logic as the quantum data bus. We can overcome this constrain by selecting a specific normal mode for quantum logic which is decoupled from heating. However, not all motional modes are prevented from heating. In what follows we will discuss this point following Ref. [59].

Let us consider the ion crystal with an odd number $N$ of ions which consists of $N-1$ ions of mass $m$ and of a central ion of the mass $M$ defining the ratio $\zeta=M / m$. Now we can follow the lines in Sec. III and find the normal modes and frequencies of the ion string with unequal ions. We find out that (i) there are $(N-1) / 2$ axial normal modes for which the central ion does not move and corresponding eigenvectors $\mathbf{D}^{(\alpha)}$ and eigenfrequencies $\nu_{\alpha}=\omega_{z} \sqrt{\mu_{\alpha}}$ do not depend on the parameter $\zeta$. Moreover, these modes do not have a component associated with the axial COM motion. (ii) There are also other $(N+1) / 2$ modes having a component of the COM motion and coupling to any uniform electric field which causes their heating.

For very small or very large values of $\zeta$ the motional modes become degenerate and pair up (FIG. 10). From this point of view the value $\zeta \simeq 1$ seems to be suitable. It has also been calculated that those modes having the central ion at rest (neglecting gradient electric fields) do not heat at all (we refer to these as cold modes), while all other motional modes heat to some extent depending on the value of $\zeta$. Their heating rate (average number of phonons gained per second) drops rapidly for $\zeta \rightarrow 1$ (FIG.11). We refer to these modes as hot modes.

Thus, it seems that the optimal choice is $\zeta \simeq 1$. That means the central (cooling) ion should be chosen such that it is identical to $N-1$ other (logic) ions or is an isotope of logic ions. For $\zeta \simeq 1$ we can choose the lowest cold mode (the second lowest motional mode called breathing mode) to be used for quantum logic as the quantum data bus because in the case of $\zeta \simeq 1$ only the lowest motional mode (corresponding to the COM mode for equal ions) will heat significantly and can be cooled via the central cooling ion. If the value of $\zeta$ differs very much from 1 , we have to cool all $(N+1) / 2$ hot modes via the central ion.
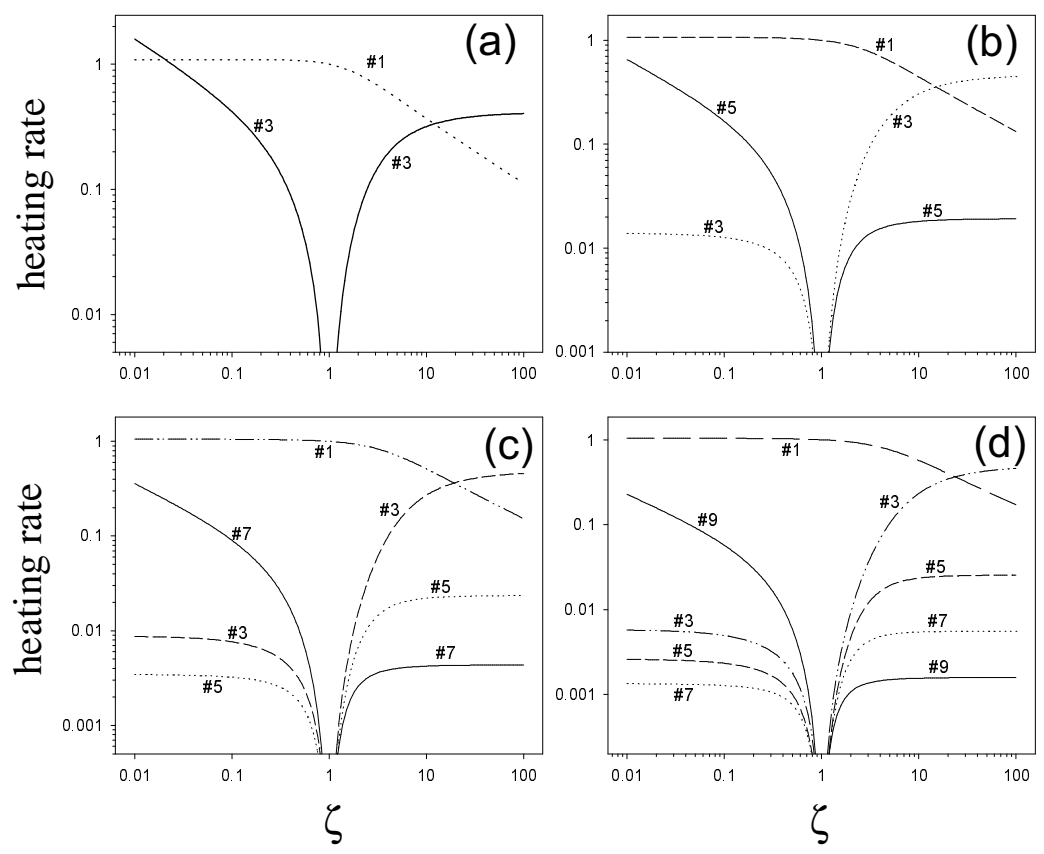

FIG. 11: Normalized heating rates for hot axial modes as a function of $\zeta$ for (a) three, (b) five, (c) seven and (d) nine ions. Used by kind permission of David Wineland and David Kielpinski [59].

The group in Garching runs experiments where the ion string consists of Indium $\left({ }^{115} \mathrm{In}^{+}\right)$and $\mathrm{Magnesium}\left({ }^{25} \mathrm{Mg}{ }^{+}\right)$ ions. The numerical analysis for the ion crystal containing these two atomic species ordered in different configuration can be found in Ref. [57]. The mass ratio is $\zeta=4.6$ which is not within the optimal range discussed above $(\zeta \simeq 1)$. On the other hand, it can quite advantageous because the heavy ion fulfills the Lamb-Dicke limit (Appendix B) easier than the light ion. Distinct atomic species can also have a very different atomic spectrum which may be found convenient when laser addressing closely spaced ions. However, for the heavy central cooling ion we pay the price in the form of heating rates of higher motional modes. By all means, Indium ions can be efficiently cooled to the ground motional state [60 and Magnesium ions can serve for quantum logic operations and storing the information.

Finally, we have to mention that the demonstration of sympathetic cooling using two different atomic species is very demanding on current experimental technology due to problems of loading the ion trap with distinct atoms in a desired configuration. 


\section{Laser cooling using electromagnetically induced transparency}

Quantum computing with cold trapped ions requires one of the motional modes (the one used as the quantum data bus) to be cooled to the motional ground state and other modes to be inside the Lamb-Dicke regime. For this purpose one could eventually use Doppler cooling assuming the axial trapping frequency $\omega_{z}$ comparable with the linewidth of the cooling transition $\Gamma$ [Eq. (5.4)]. However, it would cause very close spacing of the ions in the trap [Eq. (3.9)] with difficulties at individual addressing with the laser beam and optical resolving. On the other hand, we can use sequential sideband cooling of the motional modes described in Sec. VB. However, cooling of one motional mode causes heating of the other modes. Moreover, sideband cooling requires a very narrow bandwidth to excite the first red sideband of the respective motional mode only. Otherwise, off-resonant transitions (especially carriers) are also driven what causes heating as well [16].
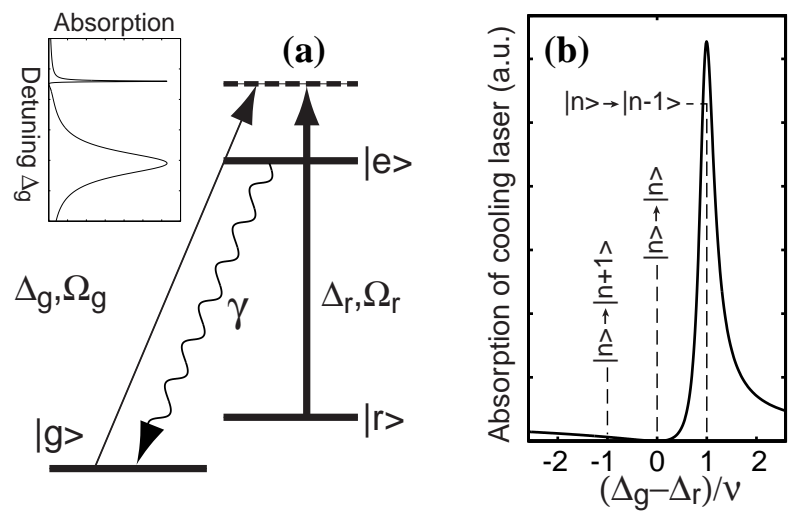

FIG. 12: (a) Levels and transitions of the cooling technique using electromagnetically induced transparency (EIT). The inset shows the absorption on $|g\rangle \leftrightarrow|e\rangle$ while strongly driving the transition $|r\rangle \leftrightarrow|e\rangle$. (b) Absorption on the carrier $(|n\rangle \rightarrow|n\rangle)$ and on the first sidebands $(|n\rangle \rightarrow|n \pm 1\rangle)$. Used by kind permission of Giovanna Morigi and Jürgen Eschner 61.

A novel cooling technique was developed in 2000 with a lower cooling limit than Doppler cooling and with a wider cooling bandwidth than sideband cooling. It was named laser cooling using electromagnetically induced transparency (EIT) 61, 62 . It is based on a quantum interference effect called EIT or coherent population trapping or also dark resonance 63. It employes a three-level system with a ground state $|g\rangle$, a stable or metastable state $|r\rangle$ and an excited state $|e\rangle$ [FIG.12(a)]. The transition $|r\rangle \leftrightarrow|e\rangle$ is driven with a laser beam of the intensity $I_{r}$ blue detuned by $\Delta_{r}$. The transition $|g\rangle \leftrightarrow|e\rangle$ is coupled by a weak laser with the intensity $I_{g}$ (where $I_{r} / I_{g} \simeq 100$ ) also blue detuned by $\Delta_{g}$. The intense laser with $\left|\Omega_{r}\right|^{2} \propto I_{r}$ introduces a significant Stark light shift $\Delta \omega$ where 64

$$
\Delta \omega=\left(\sqrt{\Delta_{r}^{2}+\left|\Omega_{r}\right|^{2}}-\left|\Delta_{r}\right|\right) / 2
$$

Thus, the laser on the transition $|r\rangle \leftrightarrow|e\rangle$ designs the absorption spectrum seen by the weak laser on $|r\rangle \leftrightarrow|e\rangle$ via the level $|e\rangle$. Then there is a broad resonance at $\Delta_{g} \simeq 0$, a dark resonance (EIT) at $\Delta_{g}=\Delta_{r}$ and a bright narrow resonance at $\Delta_{g}=\Delta_{r}+\Delta \omega$ [see the inset in FIG. 12.(a)]. Therefore, (i) taking into account also the motional degrees of freedom, (ii) setting the detunings such that $\Delta_{g}=\Delta_{r}$ and (iii) setting the Stark light shift equal to the vibrational frequency $(\Delta \omega \simeq \nu)$ we obtain the absorption spectrum depicted in FIG. 12(b). We see that the absorption on the first red sideband (cooling transition) is enhanced while the absorption on the carrier (heating transition) is eliminated.

The bright resonance width can be wide enough to cover several motional modes which can be consecutively cooled at once. It was experimentally demonstrated in Innsbruck on two motional modes separated in the frequency by $1.73 \mathrm{MHz}$. The modes were cooled to their ground motional states with $74 \%(\langle n\rangle \simeq 0.35)$ and $58 \%(\langle n\rangle \simeq 0.72)$ occupation [62]. A great improvement of these results should possible for a rebuilt apparatus allowing optimal access of laser beams to the ions 65 .

Following the advantages of laser cooling using EIT it has been estimated that all $3 N$ motional modes can be cooled to a mean phonon number $\langle n\rangle<1$ for $\omega_{z} / 2 \pi=700 \mathrm{kHz}$ and $N=10$ [63]. It is very important to cool $3 N-1$ spectator motional modes to the Lamb-Dicke regime. Otherwise, thermally excited spectator modes cause the fractional fluctuations (blurring) in the Rabi frequency of the mode used as the quantum data bus for quantum logic operations [22]. These fluctuations in the Rabi frequency of the $\alpha$ th mode can be estimated as [66]

$$
\left(\frac{\Delta \Omega}{\Omega}\right)_{\alpha} \approx \sqrt{\sum_{\beta \neq \alpha} \eta_{\beta}^{4}\left\langle n_{\beta}\right\rangle\left(\left\langle n_{\beta}\right\rangle+1\right)},
$$


where $\eta_{\beta}$ is the Lamb-Dicke parameter of the $\beta$ th motional mode and $\left\langle n_{\beta}\right\rangle$ is the respective average phonon number. The ratio $(\Omega / \Delta \Omega)$ determines the maximal number of Rabi cycles 17 . A detailed description of experimental laser cooling using EIT on Calcium ions can be found in Ref. 63.

\section{ELECTRON SHELVING}

Electron shelving is the experimental method for the discrimination between two electronic levels with an efficiency approaching 100\%. It was firstly demonstrated in 1986 [67. Let us assume a three-level atom consisting of a ground level $|g\rangle$, a metastable excited state $|e\rangle$ and an auxiliary excited fast decaying state $|r\rangle$ (FIG. 13). The $|g\rangle \leftrightarrow|e\rangle$

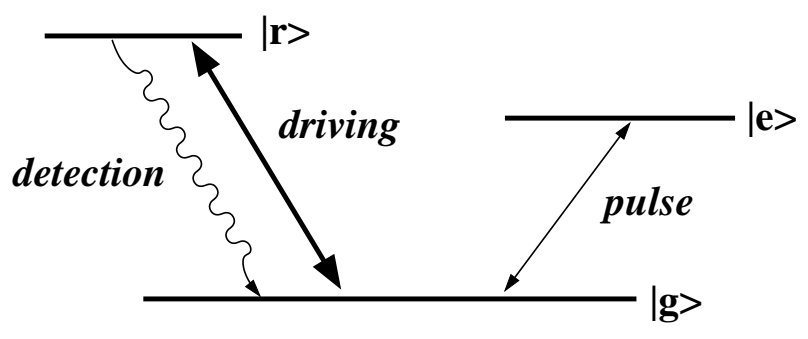

FIG. 13: Electron shelving. The $|g\rangle \leftrightarrow|e\rangle$ transition is coupled with a laser pulse forming the superposition $\alpha|g\rangle+\beta|e\rangle$. The $|g\rangle \leftrightarrow|r\rangle$ transition is driven with a strong laser. The fluorescence detection signal is collected on the $|g\rangle \leftrightarrow|r\rangle$ transition if the ion collapses to the state $|g\rangle$. If the ion is shelved in the dark state $|e\rangle$, no fluorescence is observed because the $|e\rangle$ state is a metastable state.

transition is coupled by a weak laser forming a superposition $\alpha|g\rangle+\beta|e\rangle$, while the $|g\rangle \leftrightarrow|r\rangle$ transition is driven with a strong laser. If the atom collapses to the $|g\rangle$ state during the measurement, a strong fluorescence signal is collected on the fast transition $|g\rangle \leftrightarrow|r\rangle$, i.e. the atom is excited from $|g\rangle$ to $|r\rangle$ and spontaneously decays back to the $|g\rangle$ state what is observed as the fluorescence. However, if the atom stays shelved in the metastable excited state $|e\rangle$, no fluorescence can be observed on the driven $|g\rangle \leftrightarrow|r\rangle$ transition. Hence the name electron shelving. Even though the detection efficiency is low, we can keep exciting the measuring transition $|g\rangle \leftrightarrow|r\rangle$ and detect some spontaneously emitted photons. Thus, we are able to discriminate the $|g\rangle$ and $|e\rangle$ states with almost $100 \%$ efficiency. We can also obtain the occupation probability $|\alpha|^{2}$ for the $|g\rangle$ state and $|\beta|^{2}$ for the $|e\rangle$ state averaging over many repetitions of the same experiment [15].

In the case of Calcium ions, the ground state $\left|g=S_{1 / 2}\right\rangle$ and and the metastable excited state $\left|e=D_{5 / 2}\right\rangle$ form the qubit [14. The auxiliary state corresponds to the $P_{1 / 2}$ level. The $S_{1 / 2} \leftrightarrow D_{5 / 2}$ transition is illuminated with a weak laser pulse on $729 \mathrm{~nm}$ and the $S_{1 / 2} \leftrightarrow P_{1 / 2}$ transition is driven with a strong laser at $397 \mathrm{~nm}$. However, the ion can decay from the $P_{1 / 2}$ level with a small probability to the $D_{3 / 2}$ level. Therefore, there is pumping on the $P_{1 / 2} \leftrightarrow D_{3 / 2}$ transition at $866 \mathrm{~nm}$ (see FIG. Đ).

To clarify the efficiency of the electron shelving method we report briefly some results measured in Innsbruck [15. When the ion is found in the $S_{1 / 2}$ state it scatters about 2000 photons in $100 \mathrm{~ms}$ to the detector. However, for the ion in the dark $D_{5 / 2}$ state the number of events drops to only about 150 photons in 100 ms. These 150 photons appear due to dark counts of the photomultiplier and some scattered light from the laser at $397 \mathrm{~nm}$. The ion string in the linear ion trap may represent a quantum register, where the internal state of each ion, i.e. the state of the qubit, can be detected using a CCD camera. Then the ion in the $\left|g=S_{1 / 2}\right\rangle$ state appears as a bright spot or a dark spot if the ion is found in the $\left|e=D_{5 / 2}\right\rangle$ state $13,14,15$.

\section{QUANTUM GATES}

One of the requirements for the physical implementation of quantum computing in a certain quantum system is a set of quantum gates that can be realized in the quantum system under consideration. It has been shown that any unitary operation can be composed of single-qubit rotations and two-qubit controlled-NOT gates [7]. In what follows we will describe how these and some more complex quantum gates can be implemented on cold trapped ions. We will use the notation $|g\rangle$ and $|e\rangle$ of the logical states for the qubit rather than $|0\rangle$ and $|1\rangle$ due to the representation of the qubit by the internal states of the ion. 


\section{A. Single-qubit rotations}

A general single-qubit gate corresponds to a unitary evolution operator that acts on a single qubit and is represented in the basis $\{|g\rangle,|e\rangle\}$ by the matrix

$$
W=\left(\begin{array}{ll}
W_{g g} & W_{g e} \\
W_{e g} & W_{e e}
\end{array}\right)
$$

A special case of the single-qubit gates is a single-qubit rotation (FIG. 14). Its parameterization depends on the choice of the coordinates on the Bloch sphere. We will define it in the matrix form in the basis $\{|g\rangle,|e\rangle\}$ as follows

$$
\mathcal{R}(\theta, \phi)=\left(\begin{array}{ll}
\mathcal{R}_{g g} & \mathcal{R}_{g e} \\
\mathcal{R}_{e g} & \mathcal{R}_{e e}
\end{array}\right)=\left(\begin{array}{cc}
\cos (\theta / 2) & e^{i \phi} \sin (\theta / 2) \\
-e^{-i \phi} \sin (\theta / 2) & \cos (\theta / 2)
\end{array}\right)
$$

where $\theta$ refers to the rotation and $\phi$ to the relative phase shift of the states $|g\rangle$ and $|e\rangle$ in the corresponding Hilbert space.

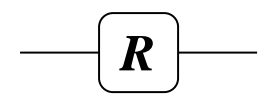

FIG. 14: Schematical representation of a single-qubit rotation. $R$ is defined by Eq. (7.2) in the basis $\{|g\rangle,|e\rangle\}$.

The single-qubit rotation can be performed on a selected ion from the ion string in the Lamb-Dicke regime by applying the unitary evolution operator 4.30). We may rewrite this operator to the form

$$
\begin{aligned}
\hat{\mathcal{A}}_{j}^{\ell}\left(\phi_{j}\right) & =\sum_{n=0}^{\infty} \cos (\ell \pi / 2)\left[\left(\left|e_{j}\right\rangle\left\langle e_{j}|\otimes| n\right\rangle\langle n|\right)+\left(\left|g_{j}\right\rangle\left\langle g_{j}|\otimes| n\right\rangle\langle n|\right)\right] \\
& +\sum_{n=0}^{\infty} \sin (\ell \pi / 2)\left[-\left(\left|e_{j}\right\rangle\left\langle g_{j}|\otimes| n\right\rangle\langle n|\right) e^{-i \phi_{j}}+\left(\left|g_{j}\right\rangle\left\langle e_{j}|\otimes| n\right\rangle\langle n|\right) e^{i \phi_{j}}\right]
\end{aligned}
$$

where we have applied the arbitrary choice of the phase factor $\left(\phi_{j} \rightarrow \phi_{j}+\pi / 2\right)$ with respect to the remark below Eq. (4.25). The operator (7.3) corresponds to the $j$ th ion illuminated with the laser beam on the carrier $\left(\omega_{L}=\omega_{0}\right)$ with the laser pulse duration $t=\ell \pi /\left|\lambda_{j}\right|$, where the laser coupling constant $\lambda_{j}$ depends on the type of (i) the driven transition and (ii) the laser configuration (see Sec. IV). We will refer to the operation expressed by Eq. (7.3) as the $\ell \pi$-pulse on the carrier.

\section{B. Two-qubit controlled-NOT gates}

A two-qubit controlled-NOT (CNOT or XOR) gate acts on two qubits denoted as a control and a target qubit (FIG. 15). If the control qubit $\left(m_{1}\right)$ is in the state $|e\rangle$, then the state of the target qubit $\left(m_{2}\right)$ is flipped. Otherwise, the gate acts trivially, i.e. as the unity operator $\mathbb{1}$. We may characterize this gate with the help of the following truth table

$$
\begin{aligned}
& \left|g_{m_{1}}\right\rangle\left|g_{m_{2}}\right\rangle \longrightarrow\left|g_{m_{1}}\right\rangle\left|g_{m_{2}}\right\rangle, \\
& \left|g_{m_{1}}\right\rangle\left|e_{m_{2}}\right\rangle \longrightarrow\left|g_{m_{1}}\right\rangle\left|e_{m_{2}}\right\rangle, \\
& \left|e_{m_{1}}\right\rangle\left|g_{m_{2}}\right\rangle \longrightarrow\left|e_{m_{1}}\right\rangle\left|e_{m_{2}}\right\rangle,
\end{aligned}
$$

The implementation of the two-qubit CNOT gate on two selected ions in the ion string requires the introduction of a third auxiliary internal level $|r\rangle$. In the original proposal [2] the selective excitation of two sublevels of the $|e\rangle$ level is used instead. The selection depends on the laser polarization, where the $|e, p=0\rangle$ and $|e, p=1\rangle$ sublevels are considered. There have also appeared proposals how to avoid the establishment of the auxiliary internal level to the scheme [68, 69, 71]. Two of them will be discussed later on in his section.

Now we are ready to write two unitary evolution operators corresponding to laser pulses driven on the first red sideband in the Lamb-Dicke regime on the $j$ th ion [Eq. 4.31)] between the internal levels $|g\rangle \leftrightarrow|e\rangle$ with the atomic 


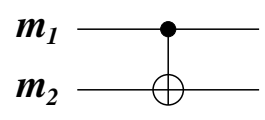

FIG. 15: Schematical representation of a two-qubit controlled-NOT (CNOT) quantum gate. The $m_{1}\left(m_{2}\right)$ qubit is control (target). The gate is defined by the truth table (7.4).

frequency $\omega_{0}^{e g}=\left(E_{e}-E_{g}\right) / \hbar$ and for $|g\rangle \leftrightarrow|r\rangle$ with $\omega_{0}^{r g}=\left(E_{r}-E_{g}\right) / \hbar$. They are given by

$$
\begin{aligned}
\hat{\mathcal{B}}_{j}^{\ell, I}\left(\phi_{j}\right) & =\cos (\ell \pi / 2)\left[\left(\left|e_{j}\right\rangle\left\langle e_{j}|\otimes| 0\right\rangle\langle 0|\right)+\left(\left|g_{j}\right\rangle\left\langle g_{j}|\otimes| 1\right\rangle\langle 1|\right)\right] \\
& -i \sin (\ell \pi / 2)\left[\left(\left|e_{j}\right\rangle\left\langle g_{j}|\otimes| 0\right\rangle\langle 1|\right) e^{-i \phi_{j}}+\left(\left|g_{j}\right\rangle\left\langle e_{j}|\otimes| 1\right\rangle\langle 0|\right) e^{i \phi_{j}}\right] \\
& +\left|g_{j}\right\rangle\left\langle g_{j}|\otimes| 0\right\rangle\langle 0|+\mathcal{O}
\end{aligned}
$$

and

$$
\begin{aligned}
\hat{\mathcal{B}}_{j}^{\ell, I I}\left(\phi_{j}\right) & =\cos (\ell \pi / 2)\left[\left(\left|r_{j}\right\rangle\left\langle r_{j}|\otimes| 0\right\rangle\langle 0|\right)+\left(\left|g_{j}\right\rangle\left\langle g_{j}|\otimes| 1\right\rangle\langle 1|\right)\right] \\
& -i \sin (\ell \pi / 2)\left[\left(\left|r_{j}\right\rangle\left\langle g_{j}|\otimes| 0\right\rangle\langle 1|\right) e^{-i \phi_{j}}+\left(\left|g_{j}\right\rangle\left\langle r_{j}|\otimes| 1\right\rangle\langle 0|\right) e^{i \phi_{j}}\right] \\
& +\left|g_{j}\right\rangle\left\langle g_{j}|\otimes| 0\right\rangle\langle 0|+\mathcal{O},
\end{aligned}
$$

where we have applied again the arbitrary choice of the phase factor $\left(\tilde{\phi}_{j}=\phi_{j}-\pi / 2 \rightarrow \phi_{j}\right)$. The symbol $\mathcal{O}$ in Eq. (7.5) and (7.6) correspond to the terms in Eq. (4.31) associated with the dynamics on higher vibrational levels for $n \geq 2$. We do not have to consider them because the ions are assumed to be cooled to the ground motional state $|n=0\rangle$. We use the Hilbert space spanned only by the motional states $|n=0\rangle$ and $|n=1\rangle$ forming an auxiliary qubit used as the quantum data bus.

The operators (7.5) and (7.6) correspond to $k \pi$-pulses on the first red sideband $\left(\omega_{L}=\omega_{0}^{e g}-\nu\right.$ and $\left.\omega_{L}=\omega_{0}^{r g}-\nu\right)$ for $n=0$ with the laser pulse duration $t=\ell \pi /\left|\lambda_{j}\right| \eta_{j}$. Finally, the two-qubit CNOT gate on two ions corresponds to the evolution operator sequence (acting from right to left) [2]

$$
\hat{\mathcal{A}}_{m_{2}}^{1 / 2}(\pi) \hat{\mathcal{B}}_{m_{1}}^{1, I} \hat{\mathcal{B}}_{m_{2}}^{2, I I} \hat{\mathcal{B}}_{m_{1}}^{1, I} \hat{\mathcal{A}}_{m_{2}}^{1 / 2}(0)
$$

where $\hat{\mathcal{A}}_{m_{2}}^{1 / 2}(0)$ and $\hat{\mathcal{A}}_{m_{2}}^{1 / 2}(\pi)$ are given by Eq. (7.3) and stand for the $\pi / 2$-pulses on the carrier $\left(\omega_{L}=\omega_{0}^{e g}\right)$ on the $m_{2}$ th ion with the phase $\phi_{j}=0$ and $\phi_{j}=\pi$, respectively. The operator $\hat{\mathcal{B}}_{m_{1}}^{1, I}$ is defined by Eq. (7.5) and represents the $\pi$-pulse on the first red sideband $\left(\omega_{L}=\omega_{0}^{e g}-\omega_{z}\right)$ on the $m_{1}$ th ion with the phase factor $\phi_{j}=0$. The operator $\hat{\mathcal{B}}_{m_{2}}^{2, I I}$ defined in Eq. (7.6) stands for the $2 \pi$-pulse on the first red sideband $\left(\omega_{L}=\omega_{0}^{r g}-\omega_{z}\right)$ on the $m_{2}$ th ion with $\phi_{j}=0$. The middle sequence $\hat{\mathcal{B}}_{m_{1}}^{1, I} \hat{\mathcal{B}}_{m_{2}}^{2, I I} \hat{\mathcal{B}}_{m_{1}}^{1, I}$ in the evolution operator $(7.7)$ can be schematically represented as follows

$$
\begin{aligned}
& \left|g_{m_{1}}\right\rangle\left|g_{m_{2}}\right\rangle|0\rangle \stackrel{\hat{\mathcal{B}}_{m_{1}}^{1, I}}{\longrightarrow} \quad\left|g_{m_{1}}\right\rangle\left|g_{m_{2}}\right\rangle|0\rangle \stackrel{\hat{\mathcal{B}}_{m_{2}}^{2, I I}}{\longrightarrow}\left|g_{m_{1}}\right\rangle\left|g_{m_{2}}\right\rangle|0\rangle \stackrel{\hat{\mathcal{B}}_{m_{1}}^{1, I}}{\longrightarrow}\left|g_{m_{1}}\right\rangle\left|g_{m_{2}}\right\rangle|0\rangle, \\
& \left|g_{m_{1}}\right\rangle\left|e_{m_{2}}\right\rangle|0\rangle \longrightarrow \quad\left|g_{m_{1}}\right\rangle\left|e_{m_{2}}\right\rangle|0\rangle \quad \longrightarrow \quad\left|g_{m_{1}}\right\rangle\left|e_{m_{2}}\right\rangle|0\rangle \longrightarrow \quad\left|g_{m_{1}}\right\rangle\left|e_{m_{2}}\right\rangle|0\rangle, \\
& \left|e_{m_{1}}\right\rangle\left|g_{m_{2}}\right\rangle|0\rangle \longrightarrow-i\left|g_{m_{1}}\right\rangle\left|g_{m_{2}}\right\rangle|1\rangle \quad \longrightarrow \quad i\left|g_{m_{1}}\right\rangle\left|g_{m_{2}}\right\rangle|1\rangle \longrightarrow\left|e_{m_{1}}\right\rangle\left|g_{m_{2}}\right\rangle|0\rangle, \\
& \left|e_{m_{1}}\right\rangle\left|e_{m_{2}}\right\rangle|0\rangle \longrightarrow-i\left|g_{m_{1}}\right\rangle\left|e_{m_{2}}\right\rangle|1\rangle \longrightarrow-i\left|g_{m_{1}}\right\rangle\left|e_{m_{2}}\right\rangle|1\rangle \longrightarrow-\left|e_{m_{1}}\right\rangle\left|e_{m_{2}}\right\rangle|0\rangle \text {. }
\end{aligned}
$$

Finally, the evolution operator (7.7) refers to the transformation

$$
\begin{aligned}
& \left|g_{m_{1}}\right\rangle\left|g_{m_{2}}\right\rangle|0\rangle \longrightarrow\left|g_{m_{1}}\right\rangle\left|g_{m_{2}}\right\rangle|0\rangle, \\
& \left|g_{m_{1}}\right\rangle\left|e_{m_{2}}\right\rangle|0\rangle \longrightarrow\left|g_{m_{1}}\right\rangle\left|e_{m_{2}}\right\rangle|0\rangle, \\
& \left|e_{m_{1}}\right\rangle\left|g_{m_{2}}\right\rangle|0\rangle \longrightarrow\left|e_{m_{1}}\right\rangle\left|e_{m_{2}}\right\rangle|0\rangle, \\
& \left|e_{m_{1}}\right\rangle\left|e_{m_{2}}\right\rangle|0\rangle \longrightarrow\left|e_{m_{1}}\right\rangle\left|g_{m_{2}}\right\rangle|0\rangle,
\end{aligned}
$$

on two selected ions labelled as $m_{1}$ and $m_{2}$ in the string of $N$ ions. 
It is evident from the discussion above that this realization of the CNOT logic gate on the ion system requires the ions to be cooled to the ground motional state $|n=0\rangle$ in order to maintain the fidelity of the computational process. Otherwise, as the ions heat up, higher terms $\mathcal{O}$ in Eq. (7.5) and $(7.6)$ also contribute and introduce significant imperfections into the implementation of the quantum gate. The two-qubit CNOT was firstly demonstrated in Boulder in 1995 [51]. A single Beryllium ion was used, where the control qubit was stored into two lowest vibrational states $|n=0\rangle$ and $|n=1\rangle$ and the target qubit was represented by two hyperfine levels $\left|g=S_{1 / 2}\left(F=2, m_{F}=2\right)\right\rangle$ and $\left|e=S_{1 / 2}\left(F=1, m_{F}=1\right)\right\rangle$.

\section{Alternative implementation of two-qubit controlled-NOT gates}

\section{Simplified quantum logic}

Monroe et al. have proposed the realization of the two-qubit quantum logic gate based on the precise setting of the Lamb-Dicke parameter [69]. The control qubit is assumed to be encoded into two lowest vibrational states $|n=0\rangle$ and $|n=1\rangle$ of the considered collective vibrational mode, while the target qubit is represented by two internal levels $\left|g_{j}\right\rangle$ and $\left|e_{j}\right\rangle$ of the $j$ th ion from the string of $N$ ions in the linear Paul trap. The CNOT gate under consideration is then described by the truth table

$$
\begin{aligned}
& |0\rangle\left|g_{j}\right\rangle \longrightarrow|0\rangle\left|g_{j}\right\rangle, \\
& |0\rangle\left|e_{j}\right\rangle \longrightarrow|0\rangle\left|e_{j}\right\rangle, \\
& |1\rangle\left|g_{j}\right\rangle \longrightarrow|1\rangle\left|e_{j}\right\rangle, \\
& |1\rangle\left|e_{j}\right\rangle \longrightarrow|1\rangle\left|g_{j}\right\rangle .
\end{aligned}
$$

Further, we adopt the main idea of the original proposal [69]. Driving the $j$ th ion with the laser on the carrier $\left(\omega_{L}=\omega_{0}\right)$ is described by the evolution operator (4.24). The coupling constant $\Omega_{j}^{n, k}$ is introduced by the expression (4.21). Then for $n=0$ and $n=1$ with $k=0$ we get

$$
\begin{aligned}
& \Omega_{j}^{0,0}=\lambda_{j} e^{-\eta_{j}{ }^{2} / 2}, \\
& \Omega_{j}^{1,0}=\lambda_{j} e^{-\eta_{j}{ }^{2} / 2}\left(1-\eta_{j}{ }^{2}\right) .
\end{aligned}
$$

Let us set the Lamb-Dicke parameter such that

$$
\eta_{j}^{2}=\frac{1}{2 p}
$$

where $p$ is an integer. The realization of the transformation (7.10) requires driving the carrier transition on the $j$ th ion with the duration $t$ such that

$$
\Omega_{j}^{0,0} t=2 p \pi
$$

We can calculate using Eq. (7.13) that

$$
\Omega_{j}^{1,0} t=(2 p-1) \pi .
$$

Substituting Eq. (7.14) and (7.15) into Eq. (4.24) we find out that the internal state of the $j$ th ion is flipped only if the collective vibrational state is $n=1\rangle$. We can write

$$
\begin{aligned}
& |0\rangle\left|g_{j}\right\rangle \longrightarrow|0\rangle\left|g_{j}\right\rangle, \\
& |0\rangle\left|e_{j}\right\rangle \longrightarrow|0\rangle\left|e_{j}\right\rangle, \\
& |1\rangle\left|g_{j}\right\rangle \longrightarrow i e^{-i \phi_{j}}|1\rangle\left|e_{j}\right\rangle, \\
& |1\rangle\left|e_{j}\right\rangle \longrightarrow i e^{+i \phi_{j}}|1\rangle\left|g_{j}\right\rangle .
\end{aligned}
$$

This transformation corresponds to the CNOT gate (7.10) apart from the phase factor $\phi_{j}$ which can be eliminated by the appropriate phase settings of subsequent operations.

The CNOT gate between distinct ions representing two logic qubits can be implemented (using the proposal being discussed) by two additional laser pulses on the first red sideband $\left(\omega_{L}=\omega_{0}-\nu\right)$. We have on mind the CNOT gate given by the truth table (7.9). Firstly, we apply a $\pi$-pulse on the first red sideband on the $m_{1}$ th ion (corresponding 
to the evolution operator (4.25) with $\Omega_{j}^{0,1} t=\pi$ ) mapping the internal state of this ion onto the collective vibrational state. We can write

$$
\begin{aligned}
& \left|g_{m_{1}}\right\rangle|0\rangle \longrightarrow\left|g_{m_{1}}\right\rangle|0\rangle, \\
& \left|e_{m_{1}}\right\rangle|0\rangle \longrightarrow-i e^{+i \tilde{\phi}_{m_{1}}}\left|g_{m_{1}}\right\rangle|1\rangle, \\
& \left|g_{m_{1}}\right\rangle|1\rangle \longrightarrow-i e^{-i \tilde{\phi}_{m_{1}}}\left|e_{m_{1}}\right\rangle|0\rangle .
\end{aligned}
$$

Secondly, we apply a laser pulse on the carrier on the $m_{2}$ th ion representing the reduced CNOT gate (7.10) and finally, we map back the collective vibrational state onto the internal state of the $m_{1}$ th ion by reapplying a $\pi$-pulse on the first red sideband on this ion. This sequence of three laser pulses corresponds to the complete CNOT gate (7.9) between two distinct ions with the appropriate choice of the phase factors.

Comparing this scheme to the original proposal of Cirac and Zoller (7.7), we need fewer laser pulses to realize a two-qubit CNOT gates on trapped ions and there is no need for a third internal auxiliary level. However, more important is the overall time needed to complete the gate and the sensitivity to imprecisions. The main limitation of this Monroe scheme is that it is slow compared with other methods at the same level of infidelity (caused by off-resonant transitions) and it is rather sensitive to imprecision in the laser intensity $\left(|\Omega|^{2} \propto I\right)[70$.

\section{Fast quantum gates}

Jonathan et al. have proposed another alternative realization of two-qubit quantum gates on cold trapped ions scalable on $N$ ions [71. It is based on (i) using carrier transitions and (ii) taking into account Stark light shifts of atomic levels. Following Ref. [71 the basic idea of fast quantum gates is that the resonant driving of a carrier transition $(|g\rangle|n\rangle \leftrightarrow|e\rangle|n\rangle)$ with an intense laser causes the splitting of dressed states $| \pm\rangle=1 / \sqrt{2}(|g\rangle \pm|e\rangle)$ in the interaction picture by amount $2 \hbar \Omega$, where the coupling constant $\Omega$ is proportional to the laser intensity $I$ [FIG. 16.(a)]. When we set the laser intensity such that the splitting of the dressed states $|-\rangle$ and $|+\rangle$ is equal to one motional

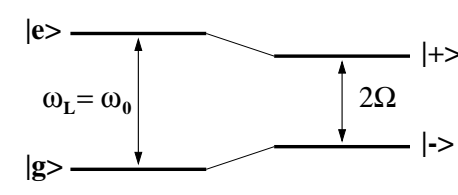

(a)

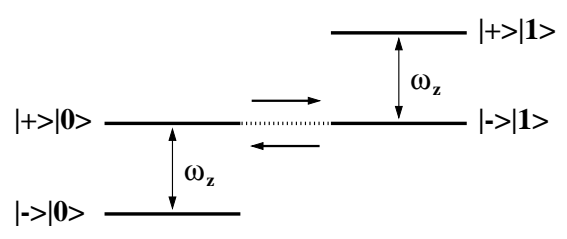

(b)

FIG. 16: Fast quantum gates on cold trapped ions are based: (a) on the splitting of the dressed states $| \pm\rangle=1 / \sqrt{2}(|g\rangle \pm|e\rangle)$ by amount proportional to the laser intensity $[\Omega=\Omega(I)]$ when the laser is tuned on the carrier transition $\left(\omega_{L}=\omega_{0}\right)$ and (b) on setting the splitting such that it is proportional to one motional quantum $(\nu=2 \Omega)$.

quantum $\hbar \nu$. Then Rabi oscillations appear between the state $|+\rangle|0\rangle$ and $|-\rangle|1\rangle$ with $|0\rangle$ and $|1\rangle$ referring to the lowest collective vibrational states of the ions [FIG. 16(b)]. Using this swapping between the $|+\rangle|0\rangle$ and $|-\rangle|1\rangle$ states one can construct a CNOT gate between two distinct ions following the truth table (7.9). Quantum gates using this idea are faster than standard quantum gates on trapped ions (discussed in Sec. VII B) approximately by the factor of $1 / \eta$ assuming the Lamb-Dicke regime. The speed of quantum gates will be discussed in Sec. IX.

\section{Multi-qubit controlled-NOT gates}

A multi-qubit controlled-NOT gate is defined by analogy to the two-qubit CNOT gate. The only difference is the number of control qubits (FIG. 17). The multi-qubit (controlled) ${ }^{q}$-NOT gate acts on $q+1$ qubits with $q$ control qubits $\left(m_{1}, \ldots, m_{q}\right)$ and the $m_{q+1}$ th qubit is target. If all control qubits are in the state $|e\rangle$, then the state of the target qubits is flipped. Otherwise, the gate acts as the unity operator $\mathbb{1}$. The truth table of the multi-qubit (controlled) ${ }^{q}$-NOT gate acting on $m_{1}, \ldots, m_{q+1}$ qubits is

$$
\begin{aligned}
& \left|\Psi_{n o}\right\rangle\left|g_{m_{q+1}}\right\rangle \longrightarrow \quad\left|\Psi_{n o}\right\rangle\left|g_{m_{q+1}}\right\rangle, \quad\left|\Psi_{n o}\right\rangle \neq \prod_{j=1}^{q} \otimes\left|e_{m_{j}}\right\rangle, \\
& \left|\Psi_{n o}\right\rangle\left|e_{m_{q+1}}\right\rangle \longrightarrow\left|\Psi_{n o}\right\rangle\left|e_{m_{q+1}}\right\rangle, \\
& \left|\Psi_{\text {yes }}\right\rangle\left|g_{m_{q+1}}\right\rangle \longrightarrow \quad\left|\Psi_{\text {yes }}\right\rangle\left|e_{m_{q+1}}\right\rangle, \quad\left|\Psi_{\text {yes }}\right\rangle=\prod_{j=1}^{q} \otimes\left|e_{m_{j}}\right\rangle, \\
& \left|\Psi_{y e s}\right\rangle\left|e_{m_{q+1}}\right\rangle \longrightarrow\left|\Psi_{y e s}\right\rangle\left|g_{m_{q+1}}\right\rangle \text {. }
\end{aligned}
$$


The multi-qubit (controlled) $)^{q}$-NOT gate acting on $q+1$ ions $\left(m_{1}, \ldots, m_{q}\right.$ ions represent the control qubits, while the $m_{q+1}$ th ion stands for the target qubit) can be realized by applying the evolution operator (acting from right to left)

$$
\hat{\mathcal{A}}_{m_{q+1}}^{1 / 2}(\pi) \hat{\mathcal{B}}_{m_{1}}^{1, I}\left[\prod_{j=2}^{q} \hat{\mathcal{B}}_{m_{j}}^{1, I I}\right] \hat{\mathcal{B}}_{m_{q+1}}^{2, I I}\left[\prod_{j=q}^{2} \hat{\mathcal{B}}_{m_{j}}^{1, I I}\right] \hat{\mathcal{B}}_{m_{1}}^{1, I} \hat{\mathcal{A}}_{m_{q+1}}^{1 / 2}(0)
$$

where the $\hat{\mathcal{B}}$ operators are taken for the value $\phi=0$. However, this choice of the phase factor has no fundamental importance. Eq. (7.19) applies for three and more ions and the scheme requires again the auxiliary qubit encoded into two lowest levels $|n=0\rangle$ and $|n=1\rangle$ of the collective vibrational mode used as the quantum data bus.

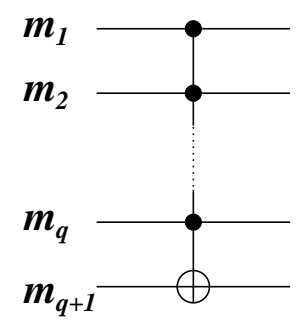

FIG. 17: Schematical representation of a multi-bit (controlled) $)^{q}$-NOT gate acting on $q+1$ qubits with $q$ control qubits and $m_{q+1}$ th qubit to be target. The gate is defined by the truth table 7.18).

Now we verify whether the evolution operator (7.19) corresponds to the truth table of the multi-qubit CNOT gate given by Eq. (7.18). At first we consider only the $\hat{\mathcal{B}}$ operators and then we comment on the action of the $\hat{\mathcal{A}}$ operators. It holds for $q+1$ ions involved in the multi-qubit CNOT gate that:

- If the $m_{1}$ th ion is in the ground state $\left|g_{m_{1}}\right\rangle$, then the action of the $\hat{\mathcal{B}}$ operators in Eq. (7.19) corresponds to the unity operator.

- If the $m_{1}$ th ion is excited with all other ions in the ground state $\left|e_{m_{1}}\right\rangle|g\rangle^{q}$, we get

$$
\left|e_{m_{1}}\right\rangle|g\rangle^{q}|0\rangle \stackrel{\hat{\mathcal{B}}_{m_{1}}^{1, I}}{\longrightarrow}-i|g\rangle^{q+1}|1\rangle \stackrel{\hat{\mathcal{B}}_{m_{2}}^{1, I I}}{\longrightarrow}-\left|r_{m_{2}}\right\rangle|g\rangle^{q}|0\rangle \stackrel{\hat{\mathcal{B}}_{m_{2}}^{1, I I}}{\longrightarrow} i|g\rangle^{q+1}|1\rangle \stackrel{\hat{\mathcal{B}}_{m_{1}}^{1, I}}{\longrightarrow}\left|e_{m_{1}}\right\rangle|g\rangle^{q}|0\rangle
$$

Thus, the transformation is performed on the $m_{1}$ th ion and then on the first next ion in the ground state. The state of all other ions in the ground state is not transformed. If more ions (besides the $m_{1}$ th one) are excited (except if they all are excited), their state does not change because the $\hat{\mathcal{B}}_{m_{j}}^{\ell, I I}$ operator acts only in the Hilbert space spanned by $\left\{\left|g_{m_{j}}\right\rangle,\left|r_{m_{j}}\right\rangle\right\}$ [see Eq. (7.6)].

- If all the ions are excited, i.e. $|e\rangle^{q+1}$, it follows that

$$
|e\rangle^{q+1}|0\rangle \stackrel{\hat{\mathcal{B}}_{m}^{1, I}}{\longrightarrow}-i\left|g_{m_{1}}\right\rangle|e\rangle^{q}|1\rangle \stackrel{\hat{\mathcal{B}}_{m}^{1, I}}{\longrightarrow}-|e\rangle^{q+1}|0\rangle .
$$

Finally, the $\hat{\mathcal{A}}$ operators complete the operation 7.19 such that it corresponds to the transformation $(7.18$ by analogy to Eq. (7.8) and (7.9).

\section{E. Multi-qubit controlled- $R$ gates}

A multi-qubit (controlled) $)^{q} R$ gate acts again on $q+1$ qubits. However, it performs a single-qubit operation (7.2) on the $m_{q+1}$ th (target) qubit if all $m_{1}, \ldots, m_{q}$ control qubits are in the state $|e\rangle$. Otherwise, it acts trivially (FIG. 18). Speaking precisely, if all control qubits are in the state $|e\rangle$, then the rotation $R=R_{1}^{\dagger} \sigma R_{2}^{\dagger} \sigma R_{2} R_{1}$ is applied (from 
right to left) on the target qubit. In the basis of the target qubit $\left\{|g\rangle_{m_{q+1}},|e\rangle_{m_{q+1}}\right\}$ we introduce the matrices

$$
\begin{array}{cc}
R=\left(\begin{array}{cc}
\cos \theta & e^{i 2 \phi} \sin \theta \\
-e^{-i 2 \phi} \sin \theta & \cos \theta
\end{array}\right), & \sigma=\left(\begin{array}{ll}
0 & 1 \\
1 & 0
\end{array}\right), \\
R_{1}=\left(\begin{array}{cc}
0 & e^{i \phi} \\
-e^{-i \phi} & 0
\end{array}\right), & R_{1}^{\dagger}=\left(\begin{array}{cc}
0 & -e^{i \phi} \\
e^{-i \phi} & 0
\end{array}\right), \\
R_{2}=\left(\begin{array}{rr}
\cos (\theta / 2) & \sin (\theta / 2) \\
-\sin (\theta / 2) & \cos (\theta / 2)
\end{array}\right), \quad R_{2}^{\dagger}=\left(\begin{array}{rr}
\cos (\theta / 2) & -\sin (\theta / 2) \\
\sin (\theta / 2) & \cos (\theta / 2)
\end{array}\right),
\end{array}
$$

where $R_{1}=\mathcal{R}(\pi, \phi), R_{1}^{\dagger}=\mathcal{R}^{\dagger}(\pi, \phi), R_{2}=\mathcal{R}(\theta, 0)$ and $R_{2}^{\dagger}=\mathcal{R}(\theta, 0)$. The rotation $\mathcal{R}(\theta, \phi)$ is defined by Eq. (7.2). The matrix $\sigma$ denotes the NOT operation. If not all control qubits are in the state $|e\rangle$, then the gate performs on the target qubit the unity operator $\mathbb{1}=R_{1}^{\dagger} \mathbb{1} R_{2}^{\dagger} \mathbb{1} R_{2} R_{1}$. Finally, we may write the truth table of the multi-qubit (controlled $)^{q}-R$ gate as follows

$$
\begin{aligned}
& \left|\Psi_{n o}\right\rangle\left|g_{m_{q+1}}\right\rangle \quad \longrightarrow \quad\left|\Psi_{n o}\right\rangle\left|g_{m_{q+1}}\right\rangle, \\
& \left|\Psi_{n o}\right\rangle\left|e_{m_{q+1}}\right\rangle \quad \longrightarrow \quad\left|\Psi_{n o}\right\rangle\left|e_{m_{q+1}}\right\rangle \\
& \left|\Psi_{y e s}\right\rangle\left|g_{m_{q+1}}\right\rangle \longrightarrow\left|\Psi_{y e s}\right\rangle\left(\cos \theta\left|g_{m_{q+1}}\right\rangle-e^{-i 2 \phi} \sin \theta\left|e_{m_{q+1}}\right\rangle\right), \\
& \left|\Psi_{\text {yes }}\right\rangle\left|e_{m_{q+1}}\right\rangle \longrightarrow\left|\Psi_{\text {yes }}\right\rangle\left(e^{i 2 \phi} \sin \theta\left|g_{m_{q+1}}\right\rangle+\cos \theta\left|e_{m_{q+1}}\right\rangle\right),
\end{aligned}
$$

where $\left|\Psi_{n o}\right\rangle$ and $\left|\Psi_{\text {yes }}\right\rangle$ are defined in Eq. (7.18). The multi-qubit controlled-R (CROT) gate (FIG. 18) is performed on cold trapped ions by applying the evolution operator (7.19) for the multi-qubit CNOT gates and the corresponding operator for the single-qubit rotations [Eq. (7.3)].

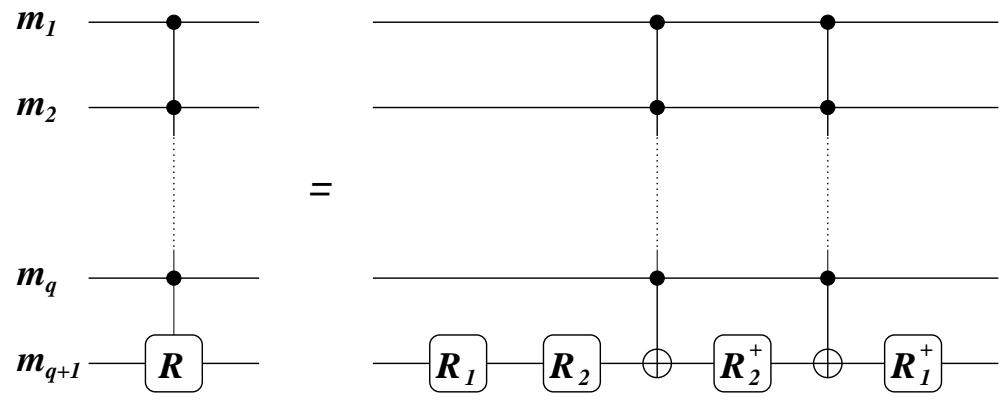

FIG. 18: Scheme of a multi-qubit (controlled) $)^{q}-R$ quantum gate. The gate acts on $q+1$ qubits with $q$ control qubits and the $m_{q+1}$ th qubit is target. $R$ is defined by Eq. 7.22 ) in the basis $\left\{|g\rangle_{m_{q+1}},|e\rangle_{m_{q+1}}\right\}$ of the target qubit. $R_{1}, R_{1}^{\dagger}, R_{2}$ and $R_{2}^{\dagger}$ are also defined by Eq. 7.22) in the same basis.

If the preparation of a particular class of quantum states does not require the introduction of a relative phase shift $\phi$ between the basis states $|g\rangle$ and $|e\rangle$, then a reduced quantum logic network is sufficient (FIG. 19). In particular, the rotation $\tilde{R}=\sigma R_{2}^{\dagger} \sigma R_{2}$ on the target qubit conditioned by the state of control qubits can be realized according to the following truth table

$$
\begin{aligned}
& \left|\Psi_{n o}\right\rangle\left|g_{m_{q+1}}\right\rangle \quad \longrightarrow \quad\left|\Psi_{n o}\right\rangle\left|g_{m_{q+1}}\right\rangle, \\
& \left|\Psi_{n o}\right\rangle\left|e_{m_{q+1}}\right\rangle \quad \longrightarrow \quad\left|\Psi_{n o}\right\rangle\left|e_{m_{q+1}}\right\rangle \\
& \left|\Psi_{\text {yes }}\right\rangle\left|g_{m_{q+1}}\right\rangle \longrightarrow\left|\Psi_{\text {yes }}\right\rangle\left(\cos \theta\left|g_{m_{q+1}}\right\rangle-\sin \theta\left|e_{m_{q+1}}\right\rangle\right), \\
& \left|\Psi_{y e s}\right\rangle\left|e_{m_{q+1}}\right\rangle \longrightarrow \quad\left|\Psi_{y e s}\right\rangle\left(\sin \theta\left|g_{m_{q+1}}\right\rangle+\cos \theta\left|e_{m_{q+1}}\right\rangle\right) .
\end{aligned}
$$

The results for the multi-qubit controlled- $R$ gates are compatible with the scheme proposed in Ref. [7], where the decomposition of multi-qubit CNOT gates into the network of two-qubit CNOT gates has been presented as well. However, this decomposition may require many elementary operations in a particular realization of quantum logic gates. It seems to be more appropriate for some practical implementations of quantum computing to implement directly multi-qubit CNOT gates (see Sec. IX). 


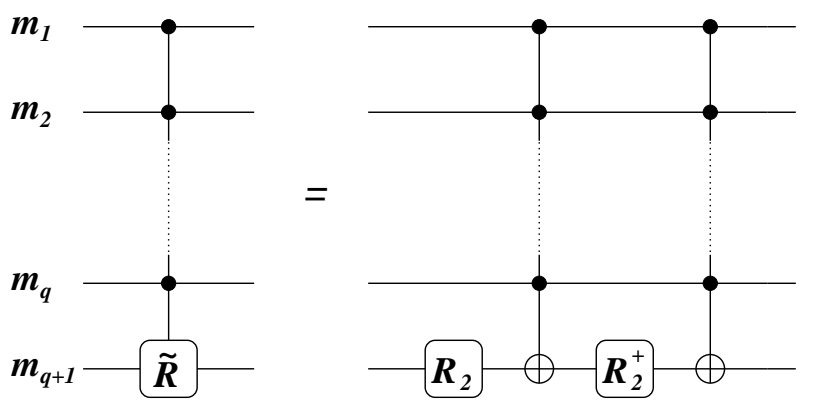

FIG. 19: Scheme of a reduced multi-qubit (controlled) $)_{-}-\tilde{R}$ quantum gate. The gate acts on $q+1$ qubits with $q$ control qubits and the $m_{q+1}$ th qubit is target. $\tilde{R}$ is defined by Eq. 7.24). $R_{2}$ and $R_{2}^{\dagger}$ are defined by Eq. 7.22) in the basis $\left\{|g\rangle_{m_{q+1}},|e\rangle_{m_{q+1}}\right\}$ of the target qubit.

\section{QUANTUM LOGIC NETWORKS}

In this section we present quantum logic networks as effective tools for the synthesis of quantum coherent superpositions of internal atomic states. We provide two particular networks, where both of them apply to an arbitrary register of qubits. The networks consist of single-qubit rotations, multi-qubit controlled-NOT and multi-qubit controlled- $R$ gates. Their implementation on cold trapped ions is described in detail in Sec.VIID and VIIE. The generation of nonclassical motional states of a trapped ion experimentally is described in Ref. [73].

We keep the notation $|g\rangle$ and $|e\rangle$ for the logical states of the qubit also in this section. Firstly, let us introduce the network for the preparation of a totally symmetric state (with respect to the permutations) of $N$ qubits, such that all qubits except one are in the excited state

$$
|\Psi\rangle=\frac{1}{\sqrt{N}}(|g e e \ldots e\rangle+|e g e \ldots e\rangle+|e e g \ldots e\rangle+\cdots+|e e e \ldots g\rangle) .
$$

It has been shown that the maximal degree of bipartite entanglement measured in the concurrence 74 is equal to $2 / N$ and is achieved when a system of $N$ qubits is prepared just in the state (8.1). The synthesis of this state realizes the network in FIG. 20 assuming all qubits to be initially prepared in the state $|e\rangle$. The rotations $Q_{j}$ are given as follows

$$
Q_{j}=\left(\begin{array}{cc}
\sqrt{\frac{N-j}{N-j+1}} & \frac{1}{\sqrt{N-j+1}} \\
-\frac{1}{\sqrt{N-j+1}} & \sqrt{\frac{N-j}{N-j+1}}
\end{array}\right), \quad j=1, \ldots, N-1 .
$$

For more details we refer to our original paper 75$]$.

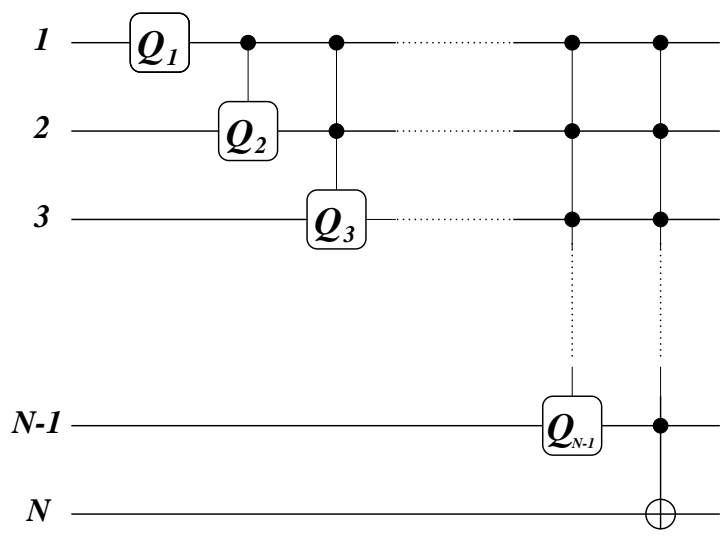

FIG. 20: The network for the synthesis of the symmetric entangled state (8.1). The rotations $Q_{j}$ are given by Eq. (8.2). $N$ qubits are assumed to be initially prepared in the state $|e e e . . . e\rangle$. 
(a)

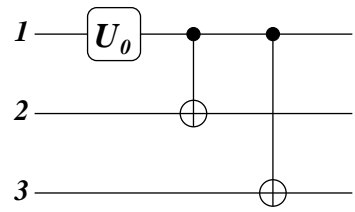

(b)

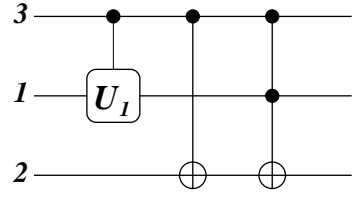

(e)

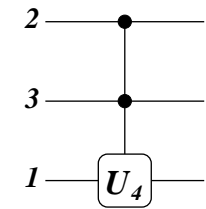

(c)

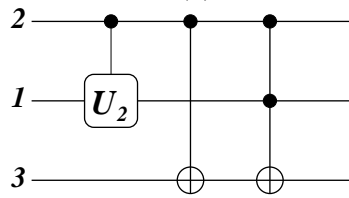

(f)

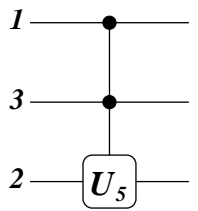

(d)

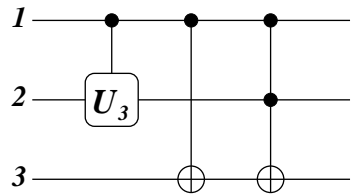

(g)

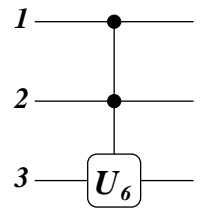

FIG. 21: An array of networks for the synthesis of an arbitrary pure quantum state 8.3 on three qubits. The initial state is $|g q g\rangle$ and the rotations $U_{j}$ are given by Eq. 8.4)-8.6. The networks (a) $-(\mathrm{g})$ generate gradually the respective terms in the superposition 8.3.

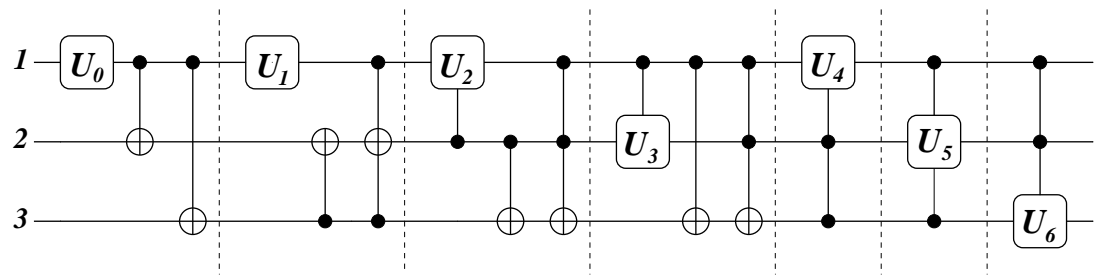

FIG. 22: A compact form of the array of the networks shown in FIG.21.

Secondly, we propose an array of quantum logic networks for the synthesis of an arbitrary pure quantum state for illustration depicted on three qubits. However, the scheme is quite easily scalable on $N$ qubits general state of three qubits in the form

$$
\begin{aligned}
|\psi\rangle & =\alpha_{0}|g g g\rangle+e^{i \varphi_{1}} \alpha_{1}|g g e\rangle+e^{i \varphi_{2}} \alpha_{2}|g e g\rangle+e^{i \varphi_{3}} \alpha_{3}|e g g\rangle \\
& +e^{i \varphi_{4}} \alpha_{4}|g e e\rangle+e^{i \varphi_{5}} \alpha_{5}|e g e\rangle+e^{i \varphi_{6}} \alpha_{6}|e e g\rangle+e^{i \varphi_{7}} \alpha_{7}|e e e\rangle,
\end{aligned}
$$

The state (8.3) can be realized by applying the array of the networks in FIG.21 (shown in a more compact form in FIG. 22) on the initial state $|g g g\rangle$, i.e. all three qubits in the state $|g\rangle$. We have denoted the rotations $U_{j}$ as follows

$$
U_{j}=\left(\begin{array}{cc}
a_{j} & e^{i 2 \phi_{j}} b_{j} \\
-e^{-i 2 \phi_{j}} b_{j} & a_{j}
\end{array}\right), \quad j=0, \ldots, 6,
$$

where $a_{j}=\cos \theta_{j}$ and $b_{j}=\sin \theta_{j}$. The state (8.3) is given by 14 real parameters and the network in FIG. 22 preparing this state is also determined by 14 parameters (seven rotations), where

$$
\phi_{0}=\frac{1}{2}\left(\pi-\varphi_{7}\right), \quad \phi_{j}=\frac{1}{2}\left(\varphi_{j}-\varphi_{7}\right), \quad j=1, \ldots, 6
$$

and

$$
b_{0}=\sqrt{1-\alpha_{0}^{2}}, \quad b_{j}=\frac{\alpha_{j}}{\sqrt{1-\sum_{k=0}^{j-1} \alpha_{k}^{2}}}, \quad j=1, \ldots, 6 .
$$

Thus, the mapping between the state under preparation (8.3) and the network (FIG. 22) is clearly defined. 


\section{SPEED OF QUANTUM GATES}

One of the most important requirements for the implementation of quantum logic (Sec. I) on a particular candidate quantum system is the physical realization of quantum gates on time scales which are much shorter compared to time scales associated with decoherence effects. We have represented quantum gates on cold trapped ions with unitary evolution operators (7.3), (7.5) and (7.6) associated with laser pulses on the carrier and on the first red sideband. However, these operators are valid only in the Lamb-Dicke and weak coupling regime. Taking into account the complete Hamiltonian (4.15) we have to deal with resonant and off-resonant transitions accompanied with Stark light shifts of the energy levels (Appendix A). A detailed treatment of this problem was presented by Steane et al. in Ref. [72 and we adopt some of their results in this section. The analysis of the speed of gate operations in ion traps was firstly discussed by Plenio and Knight in Ref. 38, 39. At first we discuss the speed of single-qubit rotations and two-qubit CNOT gates. Then we include some estimations for the speed of multi-qubit CNOT gates with cold trapped ions.

- The single-qubit rotations are associated with the transition on the carrier (7.3) with the duration $T_{\mathcal{A}}=\ell \pi /|\lambda|$, i.e. a $\ell \pi$-pulse on the carrier applied on a given ion. However, we have to consider rather the evolution operator corresponding to the Hamiltonian 4.15) when we want to discuss unwanted off-resonant transitions. Directing the laser beam such that it is perpendicular to the $z$ axis, the Lamb-Dicke parameter $\eta$ becomes equal to zero and off-resonant transitions $|e\rangle|n\rangle \leftrightarrow|g\rangle|n \pm| k|\rangle$ for $k \neq 0$ do not appear in the dynamics. Therefore, one can make the laser coupling constant $|\lambda|$ large without the restriction on the weak coupling regime characterized by the condition $|\lambda| \ll \nu$ (Appendix A). We can assume $|\lambda| / 2 \pi \simeq 300 \mathrm{kHz}$, then we get typically for a $\pi / 2$-pulse on the carrier $T_{\mathcal{A}} \simeq 1 \mu \mathrm{s}$.

- The two-qubit CNOT gate (7.7) is realized by two $\pi / 2$-pulses on the carrier $\left[\hat{\mathcal{A}}_{m_{2}}^{1 / 2}(0), \hat{\mathcal{A}}_{m_{2}}^{1 / 2}(\pi)\right]$, two $\pi$-pulses $\left(\hat{\mathcal{B}}_{m_{1}}^{1, I}\right)$ and a single $2 \pi$-pulse $\left(\hat{\mathcal{B}}_{m_{2}}^{2, I I}\right)$ on the first red sideband. A pulse on the first red sideband is represented with the unitary evolution operator (4.25) for $k=-1$. However, it was derived for an ideal case when offresonant transitions and Stark light shifts were not considered. We can correct for the light shifts by tuning the laser on the frequency $\omega_{L}=\omega_{0}-\omega_{z}+\Delta \omega$, where $\Delta \omega$ corresponds to the light shifts caused by the presence of the carrier transitions. The imprecision caused by the excitation of off-resonant transitions can be corrected by applying a correction laser pulse with a correspondingly adjusted phase. This was accomplished in Ref. 72 and the limit for the duration of the operation corresponding to the $\pi$-pulse on the first red sideband is given as

$$
\frac{1}{T_{\mathcal{B}}} \leq 2 \sqrt{2} \epsilon \sqrt{\frac{E_{r}}{N h} \frac{\omega_{z}}{2 \pi}},
$$

where $\epsilon=\sqrt{1-F}$ is the imprecision defined via the fidelity $F, E_{r}=\hbar^{2} \kappa_{\vartheta}^{2} / 2 m$ is the recoil energy of a single ion of the mass $m, \kappa=(2 \pi / \Lambda) \cos \vartheta, \Lambda$ is the laser wavelength, $h=2 \pi \hbar$ and $\omega_{z}$ is the axial trapping frequency. The limit for the duration of the operation corresponding to the $2 \pi$-pulse on the first red sideband is the double of the expression given by Eq. (9.1).

In TABLE I we give the estimations for Calcium ions ${ }^{40} \mathrm{Ca}^{+}$. We assume the angle between the laser beam and the $z$ axis to be $\vartheta=60^{\circ}$, the laser wavelength is $\Lambda=729 \mathrm{~nm}$ and the axial trapping frequency is $\omega_{z} / 2 \pi \simeq 700 \mathrm{kHz}$. Then we get the recoil frequency $E_{r} / h \simeq 2.33 \mathrm{kHz}$ and $\eta=\sqrt{E_{r} / \hbar \omega_{z}} \simeq 0.06$.

The multi-qubit CNOT on $Q$ ions (7.19) differs from the two-qubit CNOT gate only in the number of laser pulses required for its realization. The multi-qubit CNOT gate corresponds to two $\pi / 2$-pulses on the carrier $\left[\hat{\mathcal{A}}_{m}^{1 / 2}(0)\right.$, $\left.\hat{\mathcal{A}}_{m_{q+1}}^{1 / 2}(\pi)\right]$, a single $2 \pi$-pulse on the first red sideband $\left(\hat{\mathcal{B}}_{m_{q+1}}^{2, I I}\right)$ and $(2 Q-2) \pi$-pulses also on the first red sideband $\left(\hat{\mathcal{B}}_{m_{1}}^{1, I}, \hat{\mathcal{B}}_{m_{2}}^{1, I I}, \ldots, \hat{\mathcal{B}}_{m_{q}}^{1, I I}\right)$. Thus, it requires all together $2 Q+1$ laser pulses, where $Q=q+1$ refers to the number of the ions involved in the gate ( $q$ control ions, one target ion). Then, in the spirit of the previous discussion the minimal total time for the realization of the multi-qubit CNOT gate on $Q$ ions reads

$$
T=2\left(T_{\mathcal{A}}+Q T_{\mathcal{B}}\right)
$$

where we assume $T_{\mathcal{A}}=5 \mu$ s and $T_{\mathcal{B}}$ is given by Eq. (9.1) for a total number $N(Q \leq N)$ of the ions confined in the trap. We give some estimations for the realization of multi-qubit CNOT gates in TABLE I, where the number $Q$ of the ions involved in the gate and the total number $N$ of the ions in the trap are equal $(N=Q)$. We stress this point because there is a difference if we realize a two-qubit CNOT gate $(Q=2)$ having just two ions in the trap $(N=2)$, then we get for the total time of the gate

$$
T_{1}=2\left(T_{\mathcal{A}}+2 T_{\mathcal{B}}^{N=2}\right) \simeq 0.5 \mathrm{~ms}
$$




\begin{tabular}{|c|cc|cc|}
\hline & \multicolumn{2}{|c|}{$T_{\mathcal{B}}[\mu \mathrm{s}]$} & \multicolumn{2}{c|}{$T[\mathrm{~ms}]$} \\
$N$ & $F=99 \%$ & $F=75 \%$ & $F=99 \%$ & $F=75 \%$ \\
\hline \hline 2 & 124 & 24.8 & 0.50 & 0.10 \\
\hline 3 & 152 & 30.3 & 0.91 & 0.18 \\
\hline 6 & 214 & 42.9 & 2.58 & 0.52 \\
\hline 9 & 263 & 52.5 & 4.74 & 0.98 \\
\hline 10 & 277 & 55.4 & 5.55 & 1.12 \\
\hline
\end{tabular}

TABLE I: $N$ is the total number of the ions confined in the trap and also the number of the ions involved in the realization of the multiqubit CNOT gate, $T_{\mathcal{B}}$ is the duration of the operation corresponding to the $\pi$-pulse on the first red sideband given by Eq. (9.1) calculated for two values of the fidelity $(F=99 \%, F=75 \%)$ and $T$ is the total minimal time [Eq. (9.2)] for the realization of the multi-qubit CNOT gate on $N$ ions [see Eq. (7.19)] evaluated for two different fidelities.

or having a larger register of ten ions in the trap $(N=10)$, what gives

$$
T_{2}=2\left(T_{\mathcal{A}}+2 T_{\mathcal{B}}^{N=10}\right) \simeq 1.1 \mathrm{~ms}
$$

at the same fidelity $F=99 \%$.

Any multi-qubit gate on a register of size $N$ can be decomposed into a network of single-qubit rotations and twoqubit CNOT gates [7. However, there might be a possibility to realize this multi-qubit directly, if a given physical system allows it. For instance, the multi-qubit CNOT gate on six qubits can be decomposed into the network of 12 two-qubit CNOT gates including three additional auxiliary qubits [7]. In the case of cold trapped ions it requires the total time for the realization of the whole network $(Q=2, N=9)$

$$
T_{3}=12 \times 2\left(T_{\mathcal{A}}+2 T_{\mathcal{B}}^{N=9}\right) \simeq 12.7 \mathrm{~ms} .
$$

However, the direct implementation (7.19) would take only $(Q=N=6)$

$$
T_{4}=2\left(T_{\mathcal{A}}+6 T_{\mathcal{B}}^{N=6}\right) \simeq 2.6 \mathrm{~ms},
$$

which is about five-times less than the former case. We have again assumed almost the perfect fidelity $F=99 \%$ of the operation. We conclude that there can be quantum systems that may support a direct implementation of multi-qubit gates, rather than their decomposition into fundamental gates, what may bring advantages at experimental realization as well as in quantum state synthesis [75].

\section{DISCUSSION}

\section{A. Decoherence}

Throughout the paper we have discussed many aspects of cold trapped ions for quantum computing but we have not dealt with the decoherence which appears to be a main obstacle in achievements of experimental quantum computing. The reason was that our main goal has been to give a basic review and the discussion on decoherence sources and effects would refer more to an advanced study [38, 39, 76, 77, 78, 79, 80]. Nevertheless, for the sake of completeness we would like to mention on this place some decoherence aspects met in the lab. We will follow a detailed study of experimental issues in quantum manipulations with trapped ions given by Wineland et al. 22. The decoherence will be met in a more general usage of this term. Thus, by the decoherence we mean any effect that limits the fidelity (the match between desired and achieved realization). Further, we will distinguish three categories.

- Motional state decoherence is the most troublesome source of the decoherence in ion trap experiments and refers to the relaxation of two vibrational states $|n=0\rangle$ and $|n=1\rangle$ of a given motional mode used as the quantum data bus. The ions are cooled to the ground motional state $|n=0\rangle$ and the excitation to the $|n=1\rangle$ state is used for the transfer of information on a distinct ion. However, this scenario is not ideal for several reasons:

- Instability of trap parameters. We are simply not able to control all voltages as they undergo fluctuations and dephasing. 
- We also have to count on (i) the micromotion, (ii) the Coulomb repulsion between the ions making the motional modes (except the COM mode) anharmonic in reality and (iii) stray electrode fields causing possible excitations of the ion motion.

- We have considered just a single motional mode in our approach, but there are also other $3 N-1$ modes present and the cross-coupling between the modes appears. If spectator $3 N-1$ modes are not cooled to their ground motional states, the energy can be transferred to the mode of interest. This happens because the trapping potential is anharmonic in real and these higher anharmonic terms are responsible for the cross-coupling.

- We should mention also inelastic and elastic collisions with the background gas, even though experiments are carried out in an excellent environment $\left(p \simeq 10^{-8} \mathrm{~Pa}\right)$.

- Internal state decoherence corresponds to the evolution when a pure state of the ion $|\psi\rangle=\alpha|g\rangle+\beta|e\rangle$ transforms into a mixture $\hat{\rho}=|\alpha|^{2}|g\rangle\left\langle\left. g|+| \beta\right|^{2} \mid e\right\rangle\langle e|$. The ions demonstrate internal decoherence times of the order of seconds (Calcium) up to minutes and hours (Beryllium). The type of the decoherence discussed here can be eliminated by a proper choice of metastable excited states with long lifetimes.

- Operational decoherence refers to the precision of coherent laser-ion manipulations. There are several aspects that we have to consider:

- When the ion is illuminated with a laser beam, one has to control the pulse duration and the phase adjustment in order to avoid the preparation of unwanted states.

- Due to the laser spatial intensity profile, there is a probability (if the ions are spaced too closely) that the state of a neighbouring ion will be affected.

- If we consider the standing-wave configuration we have to take care of the precise position of the ion in the node or the antinode of the standing wave what seems to be very troublesome.

- Finally, off-resonant transitions are always present and we have to control the laser power very carefully to avoid their excitations.

However, there is a way to eliminate the effect of the decoherence. We can encode information into a decoherence-free subspace whose states are invariant under coupling to the environment [58, 81, 82].

\section{B. Ion trap systems}

We have been discussing cold trapped ions so far. Cold refers to the fact that all motional modes have to be cooled to their ground motional states because the dynamics assumes the precise control over the motional state. However, there have appeared other proposals referring to warm or hot trapped ions which assume an arbitrary motional state.

- Poyatos et al. 83. proposed a scheme for the realization of two-qubit CNOT gates between two trapped ions using ideas from the atomic interferometry. They split the wavepacket of the control ion into two directions depending on its internal state with a laser pulse. Then they address one of the wavepackets of the target ion changing conditionally its internal state and finally they bring together the wavepackets of the ions using another laser pulse. The ions communicate through the Coulomb repulsion and under ideal conditions the scheme is independent on the motional state of the ions.

- Milburn et al. [84] described two schemes for manipulations with warm trapped ions. Firstly, they use the adiabatic passage for the conditional phase shift, i.e. the phase of the ion is flipped if the motional mode is in the superposition of odd number states and the ion is excited. The COM mode in an arbitrary vibrational state is used for quantum logic but all other motional modes are assumed to be cooled to their ground motional states. Secondly, they apply the idea of the collective spin 85. for faster gates. This idea has been also used for the introduction of multi-qubit gates for quantum computing [86].

- Sørensen and Mølmer [87, 88] proposed a novel scheme based on the idea of bichromatic light $\left(\omega_{1}, \omega_{2}\right)$. Realizing the two-qubit CNOT gate they illuminate two ions with the bichromatic light coupling the states $|g g\rangle|n\rangle$ and $|e e\rangle|n\rangle$. They choose detunings far enough from the resonance with the first red and blue sidebands such that the intermediate states $|e g\rangle|n \pm 1\rangle$ and $|g e\rangle|n \pm 1\rangle$ are not populated in the process. The scheme is not sensitive on fluctuations of the number of phonons in the relevant motional mode. It is also possible to illuminate with the bichromatic light more ions and generate a multiparticle entangled state. Actually, these experiments were already realized in NIST [90] and they generated the GHZ state with two ions $\left|\psi_{2}\right\rangle=1 / \sqrt{2}(|g g\rangle-i|e e\rangle)$ with a 
fidelity $F=83 \%$ and also the GHZ state with four ions $\left|\psi_{4}\right\rangle=1 / \sqrt{2}(|g g g g\rangle+i|e e e e\rangle)$ with a fidelity $F=57 \%$ using Beryllium ions and the Raman scheme. Jonathan and Plenio proposed light shift induced quantum gates for trapped ions insensitive on phonon number in motional modes (thermal motion) [89].

- Finally, there has appeared a proposal of a scalable quantum computer with the ions in an array of microtraps by Cirac and Zoller [91] detailed in Ref. [92]. The ions are placed in a 2D array of independent ion microtraps [93] and there is another ion (head) that moves above this plane. If we position the head above a particular ion from the array and switch on the laser in the perpendicular direction, we can realize a two-qubit gate. This operation allows us to swap the state of the ion to the head which can be moved immediately above a distinct ion in the array and transfer the information onto it. The ions oscillating in the microtraps are not assumed to be cooled to their ground motional states. However, their motion can couple to the environment. It becomes relevant during the time when the ion interacts with the head but not in the case when the head moves.

\section{CONCLUSION}

In this paper we have tried to review achievements accomplished in the field of cold trapped ions. In the first part we have discussed in detail the ion loading and trapping process, the collective vibrational motion of the ions. We have also given a detailed derivation of the Hamiltonian governing the dynamics of the system including the discussion of weak coupling and Lamb-Dicke regime. Further, we have reviewed laser cooling techniques and a detection process with experimental illustrations on Calcium ions. In the second part we have discussed the implementation of quantum computing using cold trapped ions. In particular, we have described how to realize single-qubit, two-qubit and multiqubit quantum logic gates. Finally, we have estimated the speed of quantum gates with cold trapped ions. The aim of this paper is to give an introduction to this field with many references on relevant papers and studies.

\section{Acknowledgments}

We would like to thank Peter Knight, Danny Segal, Martin Plenio, Andrew Steane and Miloslav Dušek for their helpful comments and suggestions. We are also grateful to Rainer Blatt, Giovanna Morigi and David Kielpinski for sending us original files of their figures. This work was supported by the European Union projects QUBITS (IST1999-13021) and QUEST (HPRN-CT-2000-00121). We acknowledge the special support from the Slovak Academy of Sciences. One of us (M.Š.) is thankful for the support from the ESF via the Programme on Quantum information and quantum computation.

\section{APPENDIX A: WEAK COUPLING REGIME}

The expression (4.15) corresponds to the complete Hamiltonian in the sense that it includes also off-resonant transitions. For instance, even though a sufficiently intense laser is tuned on the carrier, the off-resonant transitions on the sidebands are also present and they cause imprecisions and perturbations in the dynamics. This can be avoided by setting the laser intensity $I \propto|\lambda|^{2}$, i.e. the laser coupling constant [Eq. (4.12) or (4.13)], sufficiently small. In what follows we will determine conditions (characterizing the weak coupling regime) under which we can neglect off-resonant transitions.

\section{Off-resonant transitions}

The implementation of quantum gates on cold trapped ions requires laser pulses on the carrier [FIG.5(a)] and on the first red sideband [FIG. F(b)]. Therefore, we will discuss the dynamics on these two spectral lines. At first, let us assume that the laser is tuned on the carrier $(\delta=0)$. The closest off-resonant transitions (detuned by the frequency $\nu$ ) are on the first blue and on the first red sideband [FIG.23(a)]. We will consider only the respective terms in the Hamiltonian (4.15) and drop down the index $j$. In the Lamb-Dicke limit we can write

$$
\hat{\mathcal{H}}_{1}=\frac{\hbar \lambda}{2} \hat{\sigma}_{+}+\frac{\hbar \lambda}{2}(i \eta) \hat{\sigma}_{+} \hat{a}^{\dagger} e^{i \nu t}+\frac{\hbar \lambda}{2}(i \eta) \hat{\sigma}_{+} \hat{a} e^{-i \nu t}+\text { H.c. }
$$

Further, we assume the ion to be initially in the state $|g\rangle|n\rangle$, we apply the Hamiltonian (A1) and calculate the probability of off-resonant transitions. The dynamics governed by a time-dependent Hamiltonian $\hat{\mathcal{H}}(t)$ is described to 
the first order by the unitary evolution operator

$$
\hat{U}\left(t, t_{0}\right) \approx \mathbb{1}-\frac{i}{\hbar} \int_{t_{0}}^{t} \hat{\mathcal{H}}\left(t^{\prime}\right) d t^{\prime} .
$$

Then the probability to find the ion (initially prepared in the state $|g\rangle|n\rangle$ ) in the state $|e\rangle|n+1\rangle$ (i.e. undergoing the off-resonant transition on the first blue sideband) is

$$
P_{B}=\left|\left\langle e\left|\left\langle n+1\left|\hat{U}_{1}\right| g\right\rangle\right| n\right\rangle\right|^{2}=\frac{|\lambda|^{2} \eta^{2}(n+1)}{\nu^{2}} \sin ^{2}\left[\frac{\nu\left(t-t_{0}\right)}{2}\right],
$$

where $\hat{U}_{1}$ is given by Eq. (A2) for the Hamiltonian (A1). The probability to find the ion in the state $|e\rangle|n-1\rangle$ corresponding to the off-resonant transition on the first red sideband is given as

$$
P_{R}=\left|\left\langle e\left|\left\langle n-1\left|\hat{U}_{1}\right| g\right\rangle\right| n\right\rangle\right|^{2}=\frac{|\lambda|^{2} \eta^{2} n}{\nu^{2}} \sin ^{2}\left[\frac{\nu\left(t-t_{0}\right)}{2}\right] .
$$

If there is no population transferred via the off-resonant transitions to the states $|e\rangle|n+1\rangle$ and $|e\rangle|n-1\rangle$, i.e. $P_{B} \ll 1$ and $P_{R} \ll 1$ at any time $t$, we can neglect these off-resonant transitions. Then we get the conditions of the weak coupling regime for the transition on the carrier in the form

$$
|\lambda| \eta \sqrt{n+1} \ll \nu
$$

and

$$
|\lambda| \eta \sqrt{n} \ll \nu .
$$

We can also avoid the off-resonant transitions by setting the laser beam perpendicular to the $z$ axis $(\vartheta=\pi / 2)$. Then the Lamb-Dicke parameter [see Eq. (4.9)] is equal to zero $\left(\eta \simeq \kappa \cos \vartheta z_{0}\right)$ and the coupling on the off-resonant transitions vanishes.

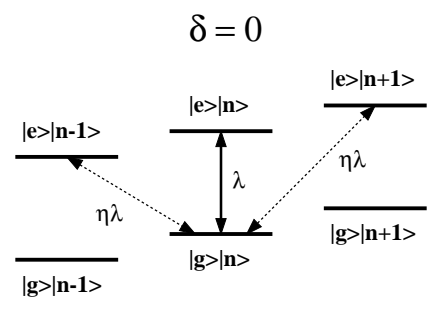

(a)

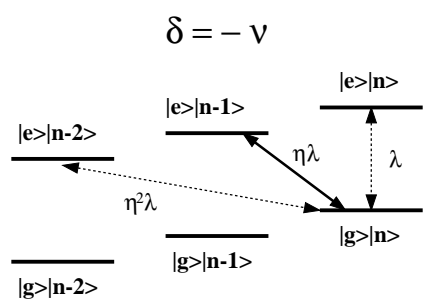

(b)

FIG. 23: (a) The laser is tuned on the carrier $(\delta=0)$ with the coupling constant $\lambda$. The off-resonant transitions are present on the first blue and red sideband with the coupling constant $\eta \lambda$. (b) The laser is tuned on the first red sideband $(\delta=-\nu)$ with the coupling constant $\eta \lambda$. The off-resonant transitions are present on the carrier with the coupling constant $\lambda$ and on the weak second red sideband $\left(\eta^{2} \lambda\right)$.

Analogically, we can assume the laser to be tuned on the first red sideband $(\delta=-\nu)$ and the closest off-resonant transitions are on the carrier and on the second red sideband [FIG.23(b)]. However, the strength of the second red sideband is of the order of $\eta^{2}$ and we can omit it in the Lamb-Dicke limit. Then the respective Hamiltonian is given as

$$
\hat{\mathcal{H}}_{2}=\frac{\hbar \lambda}{2}(i \eta) \hat{\sigma}_{+} \hat{a}+\frac{\hbar \lambda}{2} \hat{\sigma}_{+} e^{i \nu t}+\text { H.c. . }
$$

The probability to find the ion (initially in the state $|g\rangle|n\rangle$ ) in the state $|e\rangle|n\rangle$ (after the off-resonant transition on the carrier) can be calculated as

$$
P_{C}=\left|\left\langle e\left|\left\langle n\left|\hat{U}_{2}\right| g\right\rangle\right| n\right\rangle\right|^{2}=\frac{|\lambda|^{2}}{\nu^{2}} \sin ^{2}\left[\frac{\nu\left(t-t_{0}\right)}{2}\right],
$$

where $\hat{U}_{2}$ is given by Eq. A22) for the Hamiltonian (A7). We can neglect the off-resonant dynamics if $P_{C} \ll 1$ at any time $t$. Then for the weak coupling regime on the first red sideband applies

$$
|\lambda| \ll \nu .
$$

Even though the transition on the carrier is off-resonant, it has stronger coupling in the Lamb-Dicke limit than the first red sideband. Therefore, it is very important to follow in the experiment the constraint given by Eq. (A9). 


\section{Stark light shifts}

Besides the population of the off-resonant levels there is another source of imprecisions in the state manipulation. However, it is weaker and it doesn't require any special constraints on physical parameters except those for the weak coupling regime. When the laser drives a transition between two levels there appears a frequency shift called Stark light shift caused by the presence of other spectator levels. Therefore, in the experiment we have to consider the detuning (4.14) rather in the form

$$
\tilde{\delta}=\omega_{L}-\omega_{0}-\Delta \omega,
$$

where $\Delta \omega$ corresponds to the Stark light shift. The higher the laser intensity, the more significant the light shift is. We can correct for this effect by shifting the laser frequency $\left(\omega_{L} \rightarrow \omega_{L}+\Delta \omega\right)$ which tunes the transition back to the resonance. Further, we will estimate the frequency shift $\Delta \omega$.

Let us consider the Hamiltonian (4.4) and transform it to the interaction picture (4.10) with $\hat{U}_{0}=\exp \left(-i \hat{H}_{0} t / \hbar\right)$, where $\hat{H}_{0}=\left(\hbar \omega_{L} / 2\right) \hat{\sigma}_{z}$. Then we get

$$
\hat{\mathcal{H}}=\hat{\mathcal{H}}_{0}+\hat{\mathcal{V}}=\left(-\frac{\hbar \delta}{2} \hat{\sigma}_{z}+\hbar \nu \hat{a}^{\dagger} \hat{a}\right)+\left(\frac{\hbar \lambda}{2} \hat{\sigma}_{+} e^{i \eta\left(\hat{a}+\hat{a}^{\dagger}\right)}+\text { H.c. }\right)
$$

where in the Lamb-Dicke limit the interaction term reduces to

$$
\hat{\mathcal{V}} \approx \frac{\hbar \lambda}{2} \hat{\sigma}_{+}+\frac{\hbar \lambda}{2}(i \eta) \hat{\sigma}_{+} \hat{a}^{\dagger}+\frac{\hbar \lambda}{2}(i \eta) \hat{\sigma}_{+} \hat{a}+\text { H.c. }
$$

with the first term corresponding to the transition on the carrier, the second term to the first blue sideband and the last one to the transition on the first red sideband. In the second order of the time-independent perturbation theory (the first order gives no contribution) we can write for the shift of the energy levels

$$
\Delta E_{|g\rangle|n\rangle}=\sum_{\substack{m \\ \delta \neq \nu(m-n)}} \frac{\langle g|\langle n|\hat{\mathcal{V}}| e\rangle| m\rangle\langle e|\langle m|\hat{\mathcal{V}}| g\rangle| n\rangle}{\hbar \delta+\hbar \nu(n-m)}
$$

and

$$
\Delta E_{|e\rangle|n\rangle}=\sum_{\substack{m \\ \delta \neq \nu(n-m)}} \frac{\langle e|\langle n|\hat{\mathcal{V}}| g\rangle| m\rangle\langle g|\langle m|\hat{\mathcal{V}}| e\rangle| n\rangle}{-\hbar \delta+\hbar \nu(n-m)} .
$$

For instance, for the transition on the first red sideband $(\delta=-\nu)$ we can calculate

$$
\Delta E_{|g\rangle|n\rangle} \approx-\frac{\hbar|\lambda|^{2}}{4 \nu}, \quad \Delta E_{|e\rangle|n-1\rangle} \approx \frac{\hbar|\lambda|^{2}}{4 \nu}
$$

and the corresponding light shift can be estimated as

$$
\Delta \omega=\left(\Delta E_{|e\rangle|n-1\rangle}-\Delta E_{|g\rangle|n\rangle}\right) / \hbar \approx \frac{|\lambda|^{2}}{2 \nu} .
$$

If we choose $|\lambda| / 2 \pi=50 \mathrm{kHz}$ and $\nu / 2 \pi=700 \mathrm{kHz}$ so that the condition (A9) holds, then $\Delta \omega / 2 \pi \simeq 1.8 \mathrm{kHz}$.

\section{APPENDIX B: LAMB-DICKE REGIME}

In the relation for the coupling constant (4.21) we can expand the exponential function to the Taylor series about the value $\eta_{j}=0$ and use the expression (4.22) for the Laguerre polynomial. Then we get

$$
\begin{aligned}
\Omega_{j}^{n, k}(\eta) & =\lambda_{j}\left(i \eta_{j}\right)^{|k|} \sqrt{\frac{n !}{(n+|k|) !}}\left[1-\frac{\eta_{j}^{2}}{2}+\mathcal{O}\left(\eta_{j}^{4}\right)\right] \\
& \times\left[\left(\begin{array}{c}
n+|k| \\
n
\end{array}\right)-\eta_{j}^{2}\left(\begin{array}{c}
n+|k| \\
n-1
\end{array}\right)+\mathcal{O}\left(\eta_{j}^{4}\right)\right],
\end{aligned}
$$


where $\mathcal{O}\left(\eta_{j}^{4}\right)$ denotes the terms proportional to the fourth and higher powers of $\eta_{j}$. In the Lamb-Dicke regime we consider the dependence of $\Omega_{j}^{n, k}$ on the parameter $\eta_{j}$ only to its lowest order, i.e. in Eq. (B1) we neglect all terms with any higher power than $\eta_{j}^{|k|}$ and we get the coupling constant $\Omega_{j}^{n, k}$ given by the expression (4.29). This approximation can be done only if the conditions

$$
\frac{\eta_{j}^{2}}{2} \ll 1
$$

and

$$
\eta_{j}^{2}\left(\begin{array}{c}
n+|k| \\
n-1
\end{array}\right) \ll\left(\begin{array}{c}
n+|k| \\
n
\end{array}\right) \quad \Rightarrow \quad \eta_{j}^{2} \frac{n}{|k|+1} \ll 1
$$

are satisfied. We will refer to the condition $(|k|=0)$

$$
\eta_{j} \sqrt{\langle n\rangle+\frac{1}{2}} \ll 1
$$

as the Lamb-Dicke limit, where $\langle n\rangle$ is the average number of phonons in the respective vibrational mode [52].

- The Lamb-Dicke limit corresponds physically to the situation where the spatial extent of the vibrational motion of the ion $z_{0}$ is much smaller than the wavelength $\Lambda$ of the laser, where $\eta_{j} \simeq \kappa z_{0}$ and $\kappa=2 \pi / \Lambda$ [see def. in Eq. (4.9)].

- The Lamb-Dicke limit can be physically interpreted also from a different point of view. We may rewrite the Lamb-Dicke parameter of the $j$ ion of $N$ ions in the COM mode to the form $\eta_{j}^{2}=E_{r} / \hbar \omega_{z}$, where $E_{r}=\hbar^{2} \kappa^{2} / 2 m N$ is the recoil energy. It can be shown [53] that the trapped ion emits spontaneously photons of the average energy $\hbar \omega_{0}-E_{r}$, where $\omega_{0}$ is for the atomic frequency. Taking into account the Lamb-Dicke limit $\left(E_{r} \ll \hbar \omega_{z}\right)$ we may say that during the spontaneous emission the change in the vibrational state of the ion is very unlikely. In other words, the trapped ion in the Lamb-Dicke regime decays spontaneously mostly on the carrier $\left(\omega_{0} \approx \omega_{0}-E_{r} / \hbar\right)$.

- The Lamb-Dicke parameter for a single trapped ion equals to $\bar{\eta}$ and for an ion from the string of $N$ ions in the COM mode is given as $\eta_{j}=\bar{\eta} / \sqrt{N}$. It means that we can reach the Lamb-Dicke limit (B4) for $N$ ions even if the limit is not fulfilled for single ions 54 .

[1] http://www.bldrdoc.gov/timefreq/ion

[2] J.I. Cirac and P. Zoller, Quantum computation with cold trapped ions, Phys. Rev. Lett. 74, 4091 (1995)

[3] Fortschritte der Physik 48, Number 9 - 11 (2000)

[4] A. Ekert, P. Hayden, H. Inamori Basic concepts in quantum computation, quant-ph/0011013 (2000)

[5] D.P. DiVincenzo, The physical implementation of quantum computation Fortschritte der Physik 48, 771 (2000)

[6] Private communication with Daniel Loss.

[7] A. Barenco, Ch. Bennett, R. Cleve, D.P. DiVincenzo, N. Margolus, P. Shor, T. Sleator, J.A. Smolin and H. Weinfurter, Elementary gates for quantum computation, Phys. Rev. A52, 3457 (1995)

[8] V.I. Balykin, V.G. Minogin and V.S. Letokhov, Electromagnetic trapping of cold atoms, Rep. Prog. Phys. 63, 1429 (2000)

[9] W. Paul, Electromagnetic traps for charged and neutral particles Reviews of Modern Physics 62, 531 (1990)

[10] P.K. Ghosh, Ion traps (Clarendon Press, Oxford 1995)

[11] edited by J. Dalibard et al.. Fundamental systems in quantum optics (Elsevier, Amsterdam 1992)

[12] http://heart-c704.uibk.ac.at

[13] H.Ch. Nägerl, PhD thesis (Innsbruck 1998)

[14] Ch.F. Roos, PhD thesis (Innsbruck 2000)

[15] H.Ch. Nägerl, C. Roos, H. Rohde, D. Leibfried, J. Eschner, F. Schmidt-Kaler and R. Blatt, Addressing and cooling of single ions in Paul traps, Fortschritte der Physik 48, 623 (2000)

[16] D. Leibfried, C. Roos, P. Barton, H. Rohde, S. Gulde, A. Mundt, G. Reymond, M. Lederbauer, F. Schmidt-Kaler, J. Eschner and R. Blatt, Experiments towards quantum information with trapped Calcium ions, quant-ph/0009105 (2000)

[17] H. Rohde, S.T. Gulde, C.F. Roos, P.A. Barton, D. Leibfried, J. Eschner, F. Schmidt-Kaler and R. Blatt, Sympathetic ground state cooling and coherent manipulation with two-ion crystals, quant-ph/0009031 (2000)

[18] D.G. Enzer, M.M. Schauer, J.J. Gomez, M.S. Gulley, M.H. Holzscheiter, P.G. Kwiat, S.K. Lamoreaux, C.G. Peterson, V.D. Sandberg, D. Tupa, A.G. White, R.J. Hughes and D.F.V. James, Observation of power-law scaling for phase transitions in linear trapped ion crystals, Phys. Rev. Lett. 85, 2466 (2000) 
[19] A. Steane, The Ion Trap Quantum Information Processor, Appl. Phys. B64, 623 (1997)

[20] D.F.V. James, Quantum dynamics of cold trapped ions with application to quantum computation, App. Phys. B66, 181 (1998)

[21] W.H. Louisell, Quantum statistical properties of radiation (John Wiley \& Sons, New York 1973

[22] D.J. Wineland, C. Monroe, W.M. Itano, D. Leibfried, B.E. King BE and D.M. Meekhof, Experimental issues in coherent quantum-state manipulation of trapped atomic ions, Journal of Research of the National Institute of Standards and Technology 103, 259 (1998), (see also quant-ph/9710025)

[23] H.Ch. Nägerl, D. Leibfried, H. Rohde, G. Thalhammer, J. Eschner, F. Schmidt-Kaler and R. Blatt, Laser addressing of individual ions in a linear ion trap, Phys. Rev. A60, 145 (1999)

[24] D. Kielpinski, B.E. King, C.J. Myatt, C.A. Sackett, Q.A. Turchette, W.M. Itano, C. Monroe, D.J. Wineland and W.H. Zurek, Quantum Logic Using Sympathetically Cooled Ions, quant-ph/9909035 (1999)

[25] F. Schmidt-Kaler, C. Roos C, H.Ch. Nägerl, H. Rohde, S. Gulde, A. Mundt, M. Lederbauer, G. Thalhammer, T. Zeiger, P. Barton, L. Hornekaer, G. Reymond, D. Leibfried, J. Eschner and R. Blatt, Ground state cooling, quantum state engineering and study of decoherence of ions in Paul traps, Journal of Modern Optics 47, 2573 (2000)

[26] D.J. Wineland, C. Monroe, W.M. Itano, B.E. King, D. Leibfried, D.M. Meekhof, C. Myatt and C. Wood, Experimental primer on the trapped ion quantum computer. Fortschritte der Physik 46, 363 (1998)

[27] http://www.mpg.mpg.de/laserphysics.html

[28] http://researchweb.watson.ibm.com/quantuminfo

[29] http://www.lsr.ph.ic.ac.uk/iontrap

[30] http://horology.jpl.nasa.gov

[31] http://p23.lanl.gov/Quantum

[32] http://www.qubit.org/research/IonTrap

[33] http://www.ifa.au.dk/iontrapgroup

[34] http://www.physnet.uni-hamburg.de/ilp/english/research.html

[35] http://www.physik.uni-mainz.de/werth

[36] R.J. Hughes, D.F.V. James, J.J. Gomez, M.S. Gulley, M.H. Holzscheiter, P.G. Kwiat, S.K. Lamoreaux, C.G. Peterson, M.M. Sandberg, V.D. Schauer, C.M. Simmons, C.E. Thorburn, D. Tupa, P.Z. Wang and A.G. White, The Los Alamos trapped ion quantum computer experiment, Fortschritte der Physik 46, 329 (1998)

[37] M. Roberts, P. Taylor, G.P. Barwood, P. Gill, H.A. Klein and W.R.C. Rowley, Observation of an Electric Octupole Transition in a Single Ion, Phys. Rev. Lett. 78, 1876 (1997)

[38] M.B. Plenio and P.L. Knight, Realistic lower bounds for the factorization time of large numbers on a quantum computer, Phys. Rev. A53, 2986 (1996)

[39] M.B. Plenio and P.L. Knight, Decoherene limits to quantum computation using trapped ions, Proc. R. Soc. Lond. A453, 2017 (1997)

[40] J. Steinbach, J. Twamley and P.L. Knight, Engineering two-mode interactions in ion traps, Phys. Rev. A56, 4815 (1997)

[41] S.A. Gardiner, J.I. Cirac and P. Zoller, Nonclassical states and measurement of general motional observables of a trapped ion, Phys. Rev. A55, 1683 (1997)

[42] S. Chu, The manipulation of neutral particles, Reviews of Modern Physics 70, 685 (1998)

[43] C.N. Cohen-Tannoudji, Manipulating atoms with photons, Reviews of Modern Physics 70, 707 (1998)

[44] W.D. Phillips, Laser cooling and trapping of neutral atoms, Reviews of Modern Physics 70, 721 (1998)

[45] H.J. Metcalf and P. van der Straten, Laser cooling and trapping (Springer-Verlag, New York 1999)

[46] S. Stenholm, The semiclassical theory of laser cooling, Reviews of Modern Physics 58, 699 (1986)

[47] D. Bouwmeester, A. Ekert and A. Zeilinger, The Physics of Quantum Information (Springer-Verlag, Berlin 2000)

[48] G. Morigi, J.I. Cirac, K. Ellinger and P. Zoller, Laser cooling of trapped atoms to the ground state: A dark state in position space, Phys. Rev. A57, 2909 (1998)

[49] F. Diedrich, J.C. Bergquist, W.M. Itano and D.J. Wineland, Laser cooling to the zero-point energy of motion, Phys. Rev. Lett. 62, 403 (1989)

[50] B.E. King, C.S. Wood, C.J. Myatt, Q.A. Turchette, D. Leibfried, W.M. Itano, C. Monroe and D.J. Wineland, Cooling the Collective Motion of Trapped Ions to Initialize a Quantum Register, Phys. Rev. Lett. 81, 1525 (1998)

[51] C. Monroe, D.M. Meekhof, B.E. King, W.M. Itano and D.J. Wineland, Demonstration of a fundamental quantum logic gate, Phys. Rev. Lett. 75, 4714 (1995)

[52] D. Jonathan, PhD thesis (Imperial College, London 2000)

[53] C. Cohen-Tannoudji, J. Dupont-Roc and G. Grynberg, Atom-photon interactions: Basic processes and applications (John Wiley \& Sons, New York 1992)

[54] G. Morigi, J. Eschner, J.I. Cirac and P. Zoller, Laser cooling of two trapped ions: Sideband cooling beyond the Lamb-Dicke limit, Phys. Rev. A59, 3797 (1999)

[55] Eq. (14) in Ref. 54

[56] B.E. King, C.S. Wood, C.J. Myatt, Q.A. Turchette, D. Leibfried, W.M. Itano, C. Monroe and D.J. Wineland, Cooling the Collective Motion of Trapped Ions to Initialize a Quantum Register, Phys. Rev. Lett. 81, 1525 (1998)

[57] G. Morigi and H. Walther, Low temperature dynamics and laser-cooling of two-species Coulomb chains for quantum logic, quant-ph/0005082 (2000)

[58] D. Kielpinski, A. Ben-Kish, J. Britton, V. Meyer, M.A. Rowe, C.A. Sackett, W.M. Itano, C. Monroe and D.J. Wineland, Recent Results in Trapped-Ion Quantum Computing, quant-ph/0102086 (2001)

[59] D. Kielpinski, B.E. King, C.J. Myatt, C.A. Sackett, Q.A. Turchette, W.M. Itano, C. Monroe, D.J. Wineland and W.H. 
Zurek, Sympathetic cooling of trapped ions for quantum logic, Phys. Rev. A61, 032310 (2000)

[60] E. Peik, J. Abel, Th. Becker, J. von Zanthier and H. Walther, Sideband cooling of ions in radio-frequency traps, Phys. Rev. A60, 439 (1999)

[61] G. Morigi, J. Eschner and Ch. H. Keitel, Ground State Laser Cooling Using Electromagnetically Induced Transparency, Phys. Rev. Lett. 85, 4458 (2000)

[62] C.F. Roos, D. Leibfried, A. Mundt, F. Schmidt-Kaler, J. Eschner and R. Blatt, Experimental Demonstration of Ground State Laser Cooling with Electromagnetically Induced Transparency, Phys. Rev. Lett. 85, 5547 (2000)

[63] F. Schmidt-Kaler, J. Eschner, G. Morigi, C.F. Roos, D. Leibfried, A. Mundt and R. Blatt, Laser cooling with electromagnetically induced transparency: Application to trapped samples of ions or neutral atoms, quant-ph/0107087 (2001)

[64] Eq. (4) in Ref. [63]

[65] Private communication with Danny Segal.

[66] Eq. (126) in Ref. [22]

[67] W. Nagourney, J. Sandberg and H. Dehmelt, Shelved optical electron amplifier: Observation of quantum jumps, Phys. Rev. Lett. 56, 2797 (1986)

[68] A.M. Childs and I.L. Chuang, Universal quantum computation with two-level trapped ions, quant-ph/0008065 (2000)

[69] C. Monroe, D. Leibfried, B.E. King, D.M. Meekhof, W.M. Itano and D.J. Wineland, Simplified quantum logic with trapped ions, Phys. Rev. A55, R2489 (1997)

[70] Private communication with Andrew Steane.

[71] D. Jonathan, M.B. Plenio and P.L. Knight, Fast quantum gates for cold trapped ions, Phys. Rev. A62, 42307 (2000)

[72] A. Steane, Ch.F. Roos, D. Stevens, A. Mundt, D. Leibfried, F. Schmidt-Kaler and R. Blatt, Speed of ion-trap quantuminformation processors, Phys. Rev. A62, 042305 (2000)

[73] D.M. Meekhof, C. Monroe, B.E. King, W.M. Itano and D.J. Wineland, Generation of Nonclassical Motional States of a Trapped Atom, Phys. Rev. Lett. 76, 1796 (1996)

[74] W.K. Wootters, Entanglement of Formation of an Arbitrary State of Two Qubits, Phys. Rev. Lett. 80, 2245 (1998)

[75] M. Šašura and V. Bužek, Multiparticle entanglement with quantum logic networks: Application to cold trapped ions, Phys. Rev. A64, 012305 (2001)

[76] A. Garg, Decoherence in Ion Trap Quantum Computers, Phys. Rev. Lett. 77, 964 (1996)

[77] R.J. Hughes, D.F.V. James, E.H. Knill, R. Laflamme and A.G. Petschek, Decoherence Bounds on Quantum Computation with Trapped Ions, Phys. Rev. Lett. 77, 3240 (1996)

[78] D.F.V. James, Theory of Heating of the Quantum Ground State of Trapped Ions, Phys. Rev. Lett. 81, 317 (1998)

[79] Q.A. Turchette, C.J. Myatt, B.E. King, C.A. Sackett, D. Kielpinski, W.M. Itano, C. Monroe and D.J. Wineland, Decoherence and decay of motional quantum states of a trapped atom coupled to engineered reservoirs, Phys. Rev. A62, 053807 (2000)

[80] D. Kielpinski, V. Meyer, M.A. Rowe, C.A. Sackett, W.M. Itano, C. Monroe and D.J. Wineland, A decoherence-free quantum memory using trapped ions, Science 291, 1013 (2001)

[81] M.B. Plenio, S.F. Huelga, A. Beige and P.L. Knight, Cavity-loss-induced generation of entangled atoms, Phys. Rev. A59, 2468 (1999)

[82] A. Beige, S. Bose, D. Braun, S.F. Huelga, P.L. Knight, M.B. Plenio, V. Vedral, Entangling atoms and ions in dissipative environments, Journal of Modern Optics 47, 2583 (2000)

[83] J.F. Poyatos, J.I. Cirac and P. Zoller, Quantum Gates with "Hot" Trapped Ions, Phys. Rev. Lett. 81, 1322 (1998)

[84] G.J. Milburn, S. Schneider and D.F.V. James, Ion trap quantum computing with warm ions, Fortschritte der Physik 48, $801(2000)$

[85] G.J. Milburn, Simulating nonlinear spin models in an ion trap, quant-ph/9908037 (1999)

[86] X. Wang, A. Sørensen and Klaus Mølmer, Multibit Gates for Quantum Computing, Phys. Rev. Lett. 86, 3907 (2001)

[87] K. Mølmer and A. Sørensen, Multiparticle Entanglement of Hot Trapped Ions, Phys. Rev. Lett. 82, 1835 (1998)

[88] A. Sørensen and A. Sørensen, Quantum Computation with Ions in Thermal Motion, Phys. Rev. Lett. 82, 1971 (1998)

[89] D. Jonathan and M.B. Plenio, Light-shift-induced quantum gates for ions in thermal motion, quant-ph/0103140 (2001)

[90] C.A. Sackett, D. Kielpinski, B.E. King, C. Langer, V. Meyer, C.J. Myatt, M. Rowe, Q.A. Turchette, W.M. Itano, D.J. Wineland and I.C. Monroe, Experimental entanglement of four particles, Nature 404, 256 (2000)

[91] J.I. Cirac and P. Zoller, A scalable quantum computer with ions in an array of microtraps, Nature 404, 579 (2000)

[92] T. Calarco, H.J. Briegel, D. Jaksch, J.I. Cirac and P. Zoller, Quantum computing with trapped particles in microscopic potentials, Fortschritte der Physik 48, 945 (2000), (see also quant-ph/0010105)

[93] R.G. DeVoe, Elliptical ion traps and trap arrays for quantum computation, Phys. Rev. A58, 910 (1998) 